\title{
Patient-Reported Outcomes Associated with Treatments for Testicular Cancer: A Systematic Review
}

\author{
Rebecca Mercieca- \\ Bebber (D) ${ }^{\prime}$ \\ Sayeda Kamrun Naher' \\ Orlando Rincones $\mathbb{( D}^{2}$ \\ Allan Ben Smith $\mathbb{D}^{2}$ \\ Martin R Stockler (D) \\ 'NHMRC Clinical Trials Centre, The \\ University of Sydney, Camperdown, \\ NSW, Australia; ${ }^{2}$ Centre for Oncology \\ Education and Research Translation \\ (CONCERT), Ingham Institute for \\ Applied Medical Research \& University of \\ New South Wales, Liverpool, New South \\ Wales, Australia
}

Background: Testicular cancer and its treatment can have major short- and long-term effects on the health-related quality of life of those affected. This systematic review aims to summarise patient-reported outcome (PRO) data concerning health-related quality of life, functional impacts and common side-effects of treatments for testicular cancer.

Methods: We systematically searched Medline OVID, CINAHL, PubMed, Embase and the Patient-Reported Outcomes Over Time In Oncology (PROMOTION) databases from inception to 25 March 2020, using "testicular cancer" and "PRO" search terms developed in conjunction with a medical librarian. Two authors screened abstracts and full-text articles for studies that reported primary PRO data related to the treatment of testicular cancer including at least 50 participants. We excluded psychosocial data as this was included in our companion review. Data were extracted by three reviewers, and quality was assessed by two reviewers using QUAL-SYST. Studies with a quality of score over $65 \%$ were included in our narrative synthesis.

Results: A total of 1831 records were identified via our database searches and 41 met inclusion criteria. Of these, 35 included participants who had chemotherapy. Twenty-eight different PRO measures were used across the 41 studies. Of the 41 studies, 29 had quality scores over $65 \%$ and were included in our narrative synthesis. We found that chemotherapy was generally associated with a higher side-effect burden than other treatments, and higher burden was associated with higher doses of chemotherapy. Hearing problems, peripheral neuropathy, and Raynaud's phenomena were particularly common side-effects. Problems with sexual functioning were associated with chemotherapy, radiotherapy and surgery.

Discussion: While many studies found that between-treatment differences resolved within the first 12 months since diagnosis, there were many long-term and dose-dependent impacts associated with chemotherapy and radiotherapy across PRO domains. Offering information about these aspects, and information about expected survival outcomes, will help inform, prepare, and empower patients to make decisions about treatment aligned with their preferences and personal situations.

Keywords: testicular cancer, patient-reported outcomes, quality of life, cancer survivorship

\section{Introduction}

Testicular cancer is the most common cancer diagnosis among young men aged 15-40 years. ${ }^{1,2}$ Although it is a relatively rare cancer, incidence is increasing worldwide, particularly in Western Europe, Northern Europe, Australia and New Zealand. $^{2}$ Treatment for testicular cancer typically involves orchiectomy (ie, removal of the affected testicle), followed by surveillance, with or without
Correspondence: Rebecca MerciecaBebber

NHMRC Clinical Trials Centre, The University of Sydney, Locked Bag 77, Camperdown, NSW, I450, Australia Email Rebecca.mercieca@sydney.edu.au 
chemotherapy, radiotherapy or retroperitoneal lymph node dissection (RPLND), depending on the diagnosis and stage of disease, ${ }^{3}$ and patient and clinician preferences. Treatments for testicular cancer are highly effective, particularly for earlier stages of disease. Overall survival rates are high, with $94.9 \%$ living more than five years post diagnosis, and five-year survival rates have steadily been increasing since the $1970 \mathrm{~s} .{ }^{4}$ The five-year relative survival rate for localized testicular cancer, accounting for $67.8 \%$ of cases, is $99.0 \%{ }^{4}$ The health-related quality of life (HRQOL) of survivors is therefore a primary concern. Each treatment is associated with side-effects that manifest during active treatment or after completion, and can persist for years hence; sometimes for life.

For example, impaired fertility may become evident after orchiectomy. ${ }^{5,6}$ Men who have had a bilateral orchiectomy will not be able to conceive a pregnancy unless semen samples were stored prior to surgery. Neural damage following RPLND and cumulative doses of chemotherapy can also lead to fertility issues. ${ }^{7}$ Body image issues are also commonly reported following orchiectomy, due to changes in physical appearance after removal of a testicle, which can impact psychological wellbeing, selfconfidence, sexual functioning and perceptions of masculinity. ${ }^{8}$ Cisplatin-based chemotherapies have been associated with hearing loss, Raynaud's phenomena, peripheral neuropathy and cardiovascular disease.,10 Radiotherapy has been associated with gastrointestinal complaints in the long term. ${ }^{11}$ There is equivocal evidence for the impact of some treatments on survival, for example, surveillance versus a single cycle of carboplatin for the treatment of patients with Stage I disease; therefore, data about the impact of treatment side-effects is particularly important.

Given the young age of affected men, long-term sideeffects of treatments for testicular cancer have great potential to affect the HRQOL of survivors for much of their adult life. HRQOL is defined as "the subjective assessment of the impact of disease and treatment across the physical, psychological, social and somatic domains of functioning and wellbeing". ${ }^{12}$ Fertility, body image and sexual functioning concerns can be particularly concerning for men seeking a partner, or wanting to start or grow their families.

Research evidence about the impact of different treatments on aspects of functioning, HRQOL and other patient-reported outcomes (PROs) provides essential information to help newly diagnosed men decide on treatment options, and/or be prepared for the impact of their treatment. This systematic review aims to summarise PRO data concerning the impact of various treatments for testicular cancer. PROs are defined as any report about the status of a patient's health that comes directly from the patient, without interpretation by anyone else. ${ }^{13}$ We do not report psychosocial outcomes in this review, as they have been addressed in a companion paper. ${ }^{14}$

\section{Methods}

Our methods follow the Preferred Reporting Items for Systematic Reviews and Meta-Analyses (PRISMA) guidelines. $^{15}$

\section{Search Strategy}

Medline OVID, Cumulative Index to Nursing and Allied Health Literature (CINAHL), PubMed, Embase and the Patient-Reported Outcomes Over Time In Oncology (PROMOTION) (http://promotion.gimema.it/) databases were systematically searched from inception to 25 March 2020, using a search strategy developed in collaboration with an academic librarian. The search terms combined PRO and testicular cancer terms (see Appendix 1). Duplicate entries were identified in Endnote X9 and removed.

\section{Study Screening}

Two authors independently screened all abstracts according to pre-established eligibility criteria. Both authors completed a pilot-screening exercise of 200 papers to determine agreement and test the eligibility criteria before screening the remaining abstracts. Full-text papers were checked against the same eligibility criteria, with the addition of one further exclusion criterion (see below).

\section{Eligibility Criteria}

Studies were included if they reported primary PRO data related to the therapeutic treatment of testicular cancer. Studies with: samples including other cancer diagnoses, less than 50 patients, patient-reported psychosocial data only, psychosocial or complementary alternative medicine (CAM) interventions, qualitative data only, conference abstracts, research commentaries, systematic reviews, nonEnglish reports and PRO measure validation or methodological papers were excluded. At the full-text stage, we also excluded studies if PRO data were not reported by treatment received, in both single- and multi-arm studies. If a study reported psychosocial outcomes in addition to 
other PROs, the psychosocial data were excluded (as it was captured by our companion review), ${ }^{14}$ while the other relevant data were included.

\section{Data Extraction}

The following data were extracted from each publication by one of three reviewers (RMB, SKN, OR): study design, sample size, stage of disease, recruiting countries, recruitment period, participant age at diagnosis and at survey completion, primary endpoint, PRO objectives, treatments received, PRO measures used, PRO domains reported, time points with PRO data reported, summary of key PRO findings for each treatment group.

\section{Assessment of Research Quality of Included Studies}

Two reviewers (SKN, OR) assessed the quality of studies using QUAL-SYST criteria. ${ }^{16}$ Studies that scored a total of $65 \%$ or higher (of applicable items) are the focus of our review. This cut off was chosen with the intention of being inclusive, but not too liberal, based on Kmet's guide that $55 \%$ is considered "relatively liberal". ${ }^{16}$

\section{Narrative Synthesis}

Key findings comparing the PROs between treatment groups were extracted and described for this review using the narrative synthesis technique. Due to differences in the study design, measures, and analyses among the included studies, the nature of information available in each study manuscript varied, ranging from mean differences of scale scores, regressions or other multivariate comparisons, or raw scale scores for each PRO domain.

\section{Results}

A total of 1831 records were identified via our database searches, as shown in Figure 1. After duplicates were removed, 1406 abstracts were screened, and 195 full-text articles were checked. Forty-one articles met inclusion criteria (listed in Appendix 2).

\section{Studies}

Of the 41 studies, 36 described PROs associated with chemotherapy, 23 described PROs associated with radiotherapy, 15 for surveillance, 14 for RPLND, and four studies described PRO for surgery without specifying the nature of surgery. Studies recruited largely from Western Europe $(n=27)$, followed by the UK $(n=8)$, Australia/New
Zealand $(\mathrm{n}=5)$, North America $(\mathrm{n}=4)$, Asia (Japan only, $n=3)$, and Eastern Europe $(n=2)$. The most common study design was cross-sectional $(\mathrm{n}=22)$, followed by longitudinal non-randomised studies $(\mathrm{n}=8)$, randomised controlled trials $(n=5)$, case-control studies $(n=2)$, retrospective studies $(\mathrm{n}=2)$, pilot study $(\mathrm{n}=1)$ and a pooled analysis of RCT data $(\mathrm{n}=1)$.

Excluding psychosocial measures, 14 different validated PRO measures and 13 study-specific measures were used. The most commonly used PRO measures were the European Organisation for the Research and Treatment of Cancer (EORTC) Quality of Life Questionnaire Core (QLQ-C30) ${ }^{17}(\mathrm{n}=14)$, Short Form-36 $(\mathrm{SF}-36)^{18} \quad(\mathrm{n}=9), \quad$ Scale for Chemotherapy-Induced Neurotoxicity $(\mathrm{SCIN})^{19}(\mathrm{n}=6)$, EORTC QLQ-TC26 testicular cancer module ${ }^{20}(\mathrm{n}=5)$, Swedish Health-Related Quality of Life Survey (SWED-QUAL) ${ }^{21}(\mathrm{n}=2)$ and The Gothenburg Quality of Life Instrument ${ }^{22}(n=2)$. All other measures were used in just one study.

A summary of PRO findings is presented in Table 1. Due to most studies describing PROs associated with chemotherapy, we have presented the table in two groups: studies including chemotherapy and studies not including chemotherapy. Of the 41 studies, 29 scored above our QUALSYST quality score threshold of $>65 \%$, $11,19,23-48$ and PRO findings are described below. We have categorised these outcomes as they were reported in the source papers, even if some of the groupings seem arbitrary or mechanisms might be different.

\section{Neurotoxicity, Raynaud's and Related Phenomena Chemotherapy Dose-Induced Neurotoxicity and Raynaud-Like Phenomena}

Among the 26 higher quality studies including a chemotherapy treatment group, higher doses of chemotherapy were generally associated with higher selfreported neurotoxicity. ${ }^{9,19,23,24,28}$ Specifically, Oldenburg 2007 ( $\mathrm{N}=238$ ) found that cumulative dose of cisplatin was significantly associated with paraesthesia in the toes $(\mathrm{p}<0.001)$ and Raynaud-like phenomena in the toes $(\mathrm{p}<0.001)$. The risk of a more severe symptom class increased by roughly 1.3 for each step of $100 \mathrm{mg} / \mathrm{m} 2$ cumulative cisplatin, the dose corresponding to one cycle of chemotherapy. ${ }^{9}$ Similarly, Sprauten $(\mathrm{N}=169)$ reported that total SCIN score was positively associated with administered cisplatin dose $(\mathrm{p}=0.016)$, dose-intensive therapy 


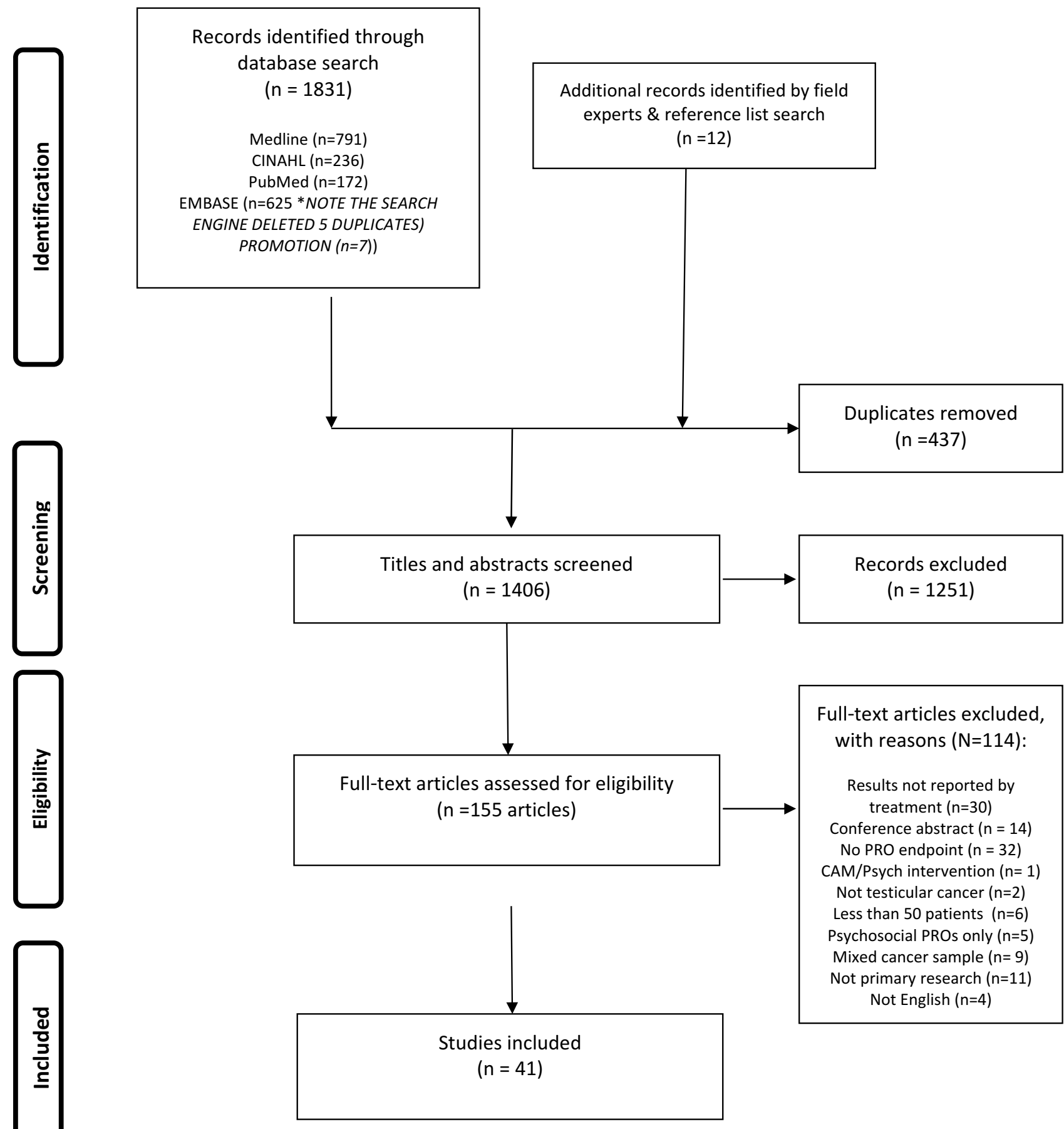

Figure I PRISMA flow diagram.

Notes: PRISMA figure adapted from Moher D, Liberati A, Tetzlaff J, Altman DG; The PRISMA Group. Preferred reporting items for systematic reviews and meta-analyses:the PRISMA statement.

PLoS Med. 2009;6(7):el000097. Creative Commons

$(\mathrm{p}=0.032)$, and age at survey $(\mathrm{p}=0.035)$ in univariate but not multivariate analyses. ${ }^{44}$ Glendenning and colleagues $(\mathrm{N}=739)$ also found that peripheral neuropathy increased significantly with increased cisplatin dose. ${ }^{28}$ In another study $(\mathrm{N}=149)$, participants randomised to 4 cycles of BEP had higher mean scores for numbness or pins and needles, compared to participants randomised to 3 cycles of BEP, at 6, 9 and 12 months post-randomisation 


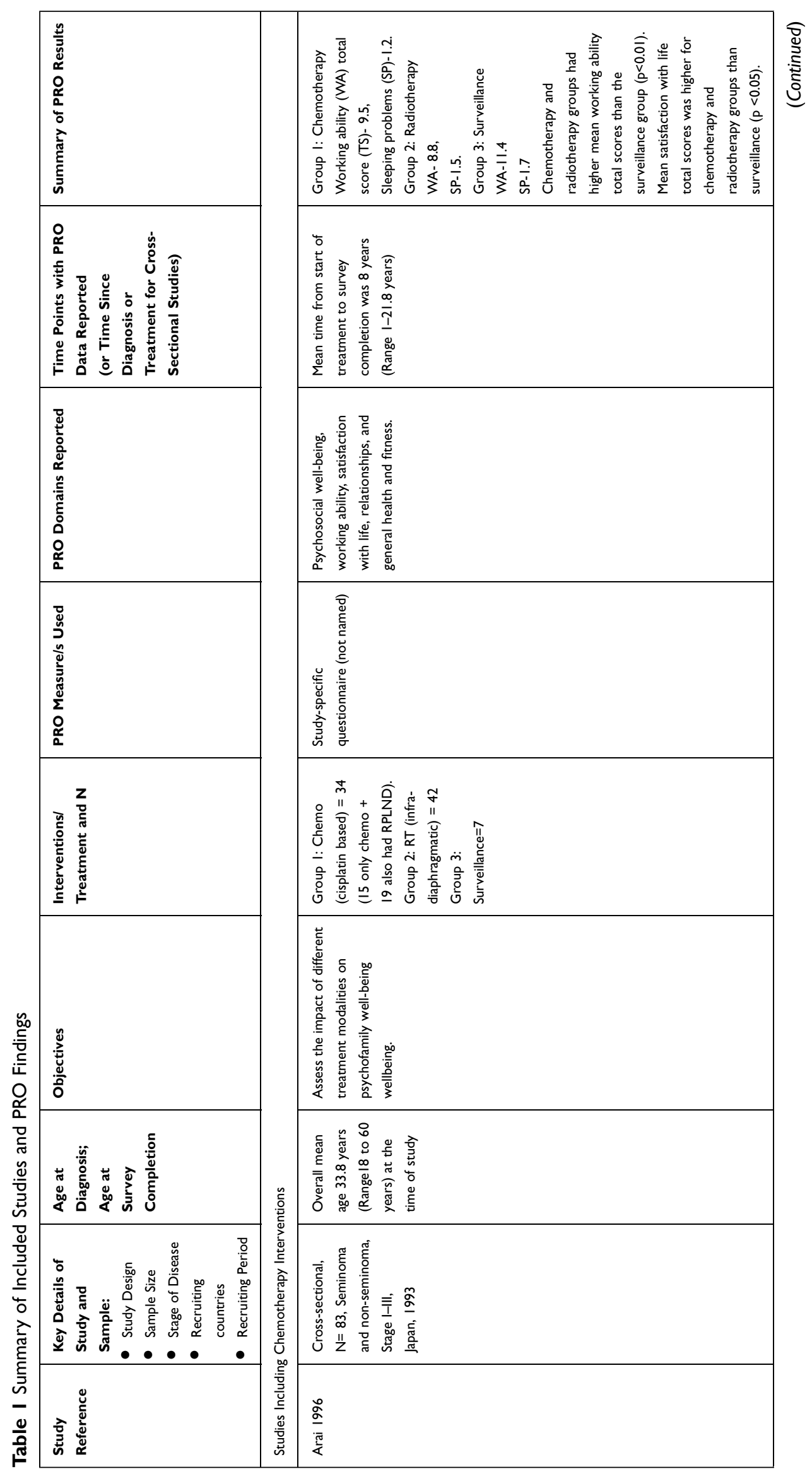




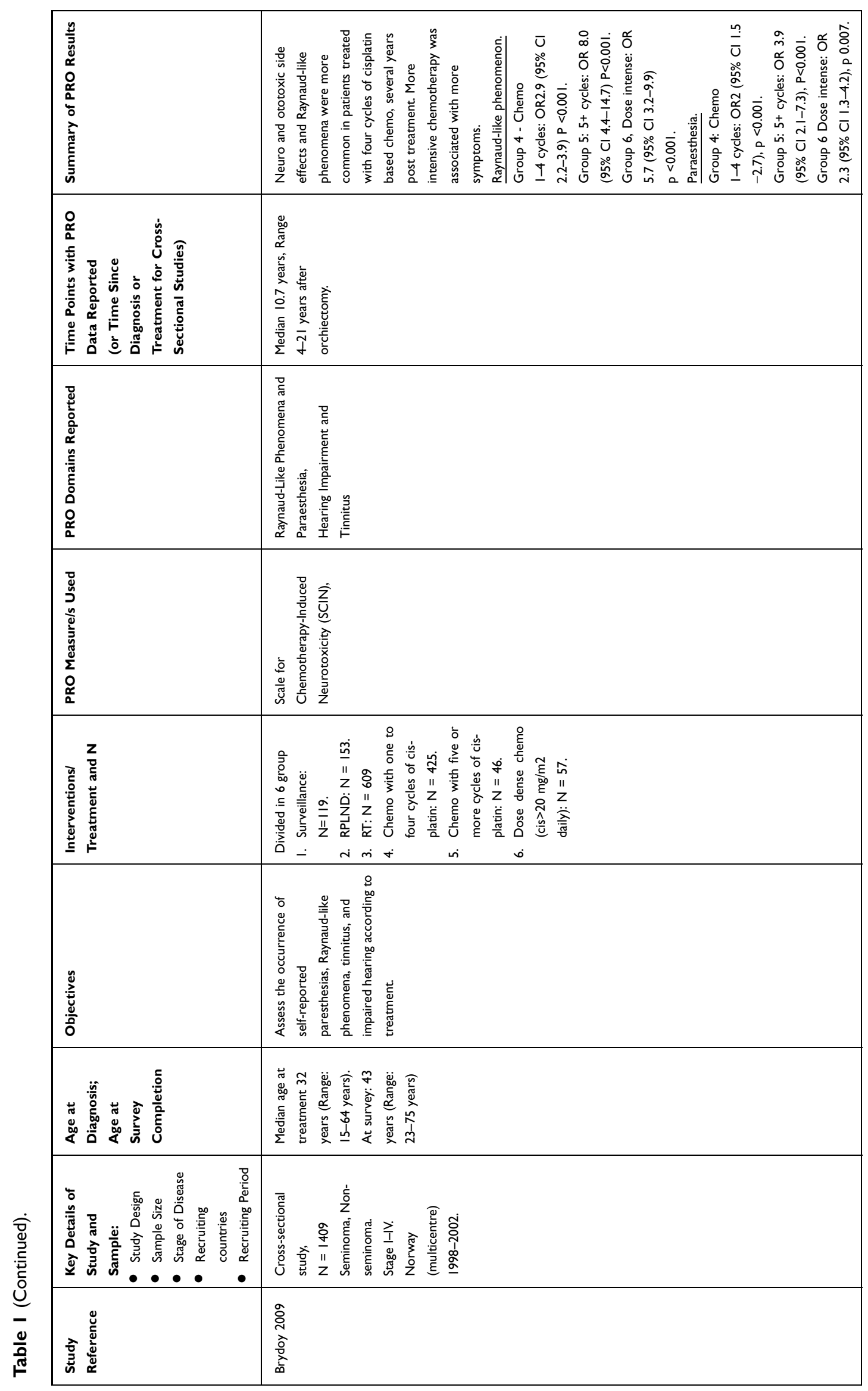




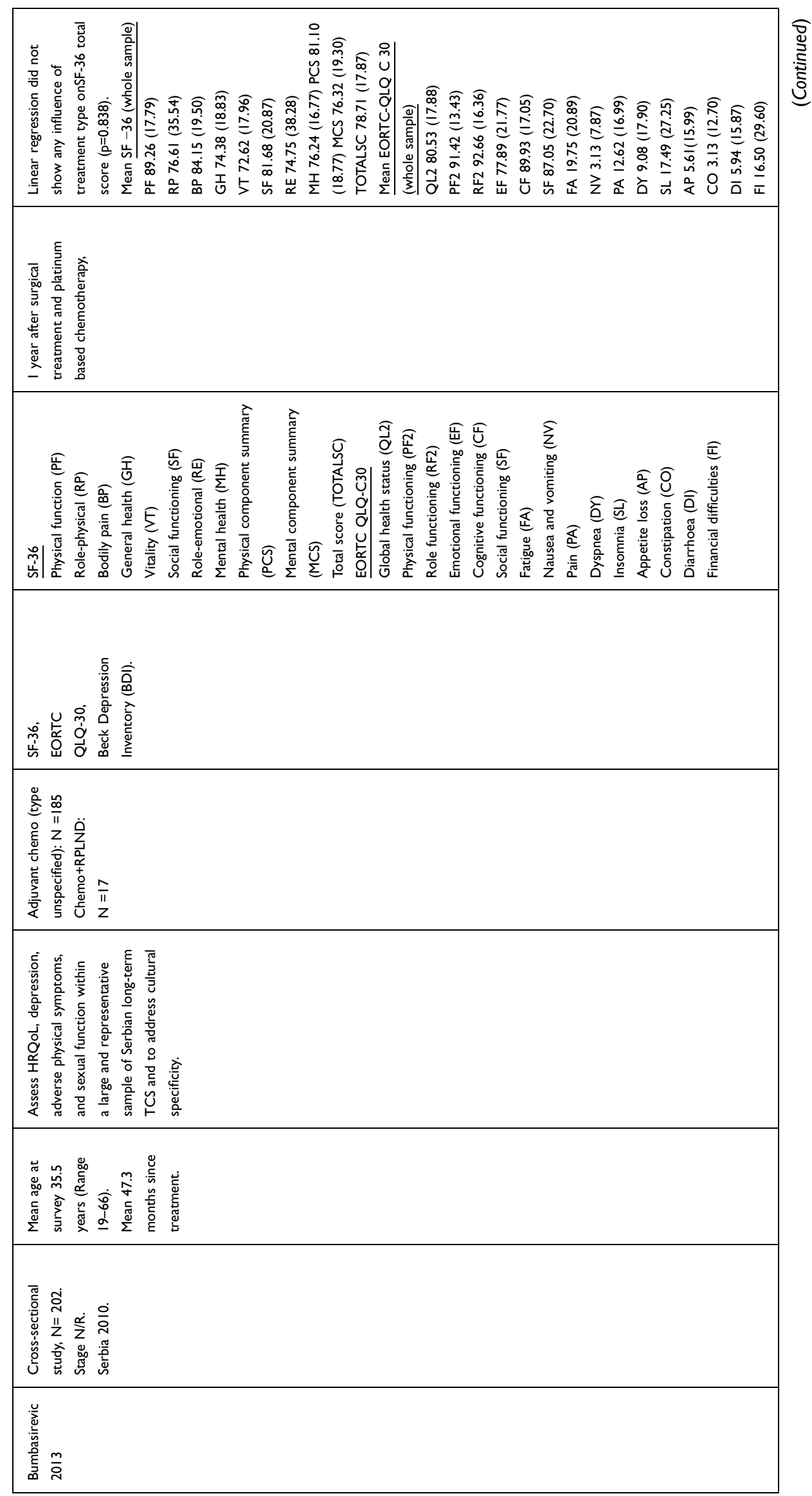




\begin{tabular}{|c|c|}
\hline 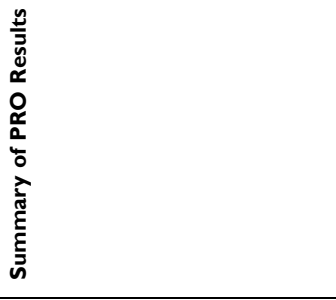 & 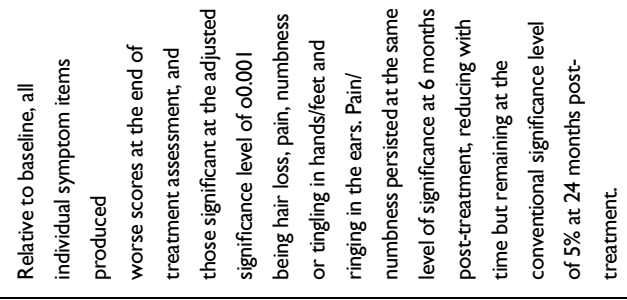 \\
\hline 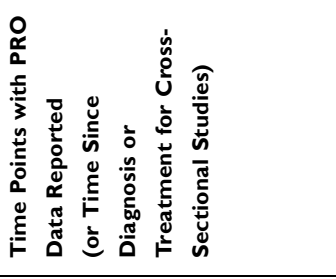 & 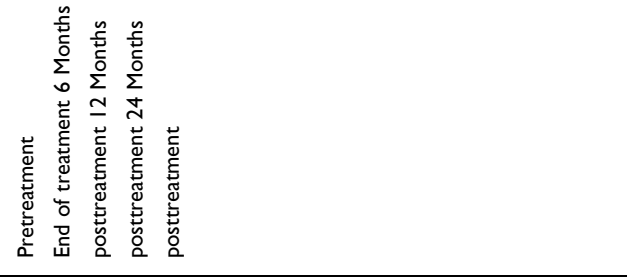 \\
\hline 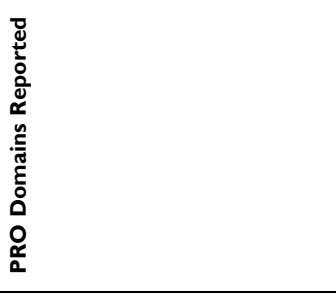 & 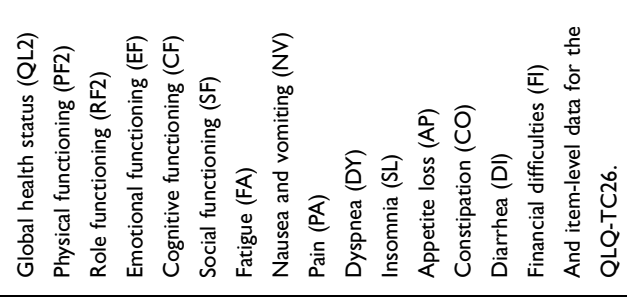 \\
\hline 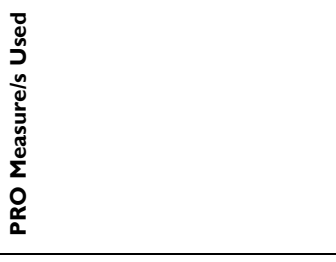 & 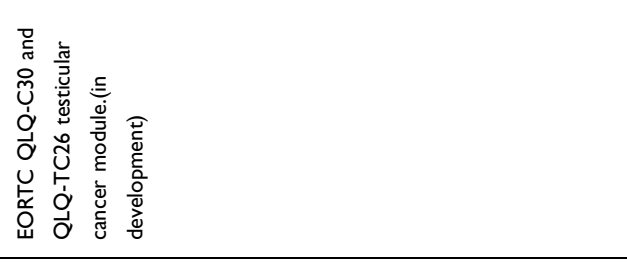 \\
\hline 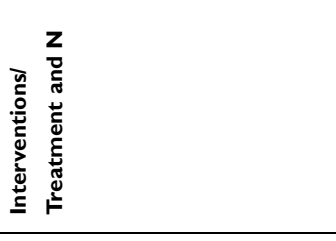 & 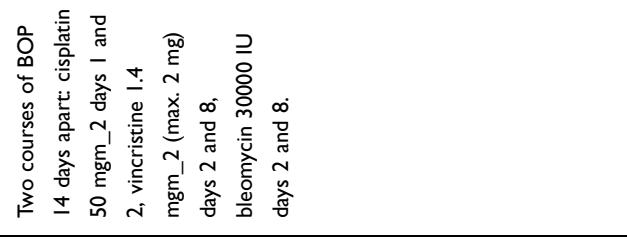 \\
\hline & 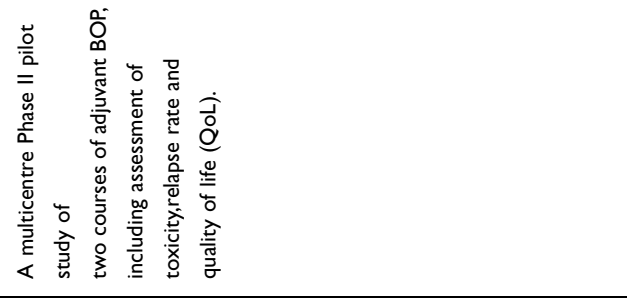 \\
\hline 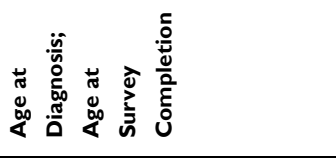 & 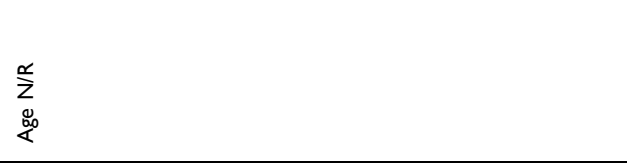 \\
\hline 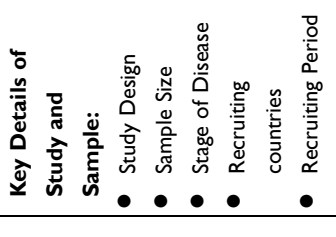 & 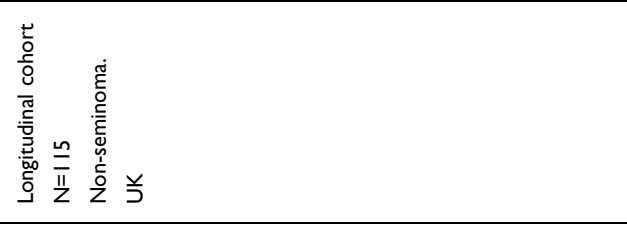 \\
\hline 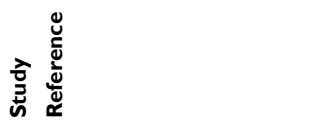 & 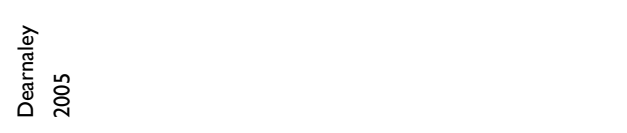 \\
\hline
\end{tabular}




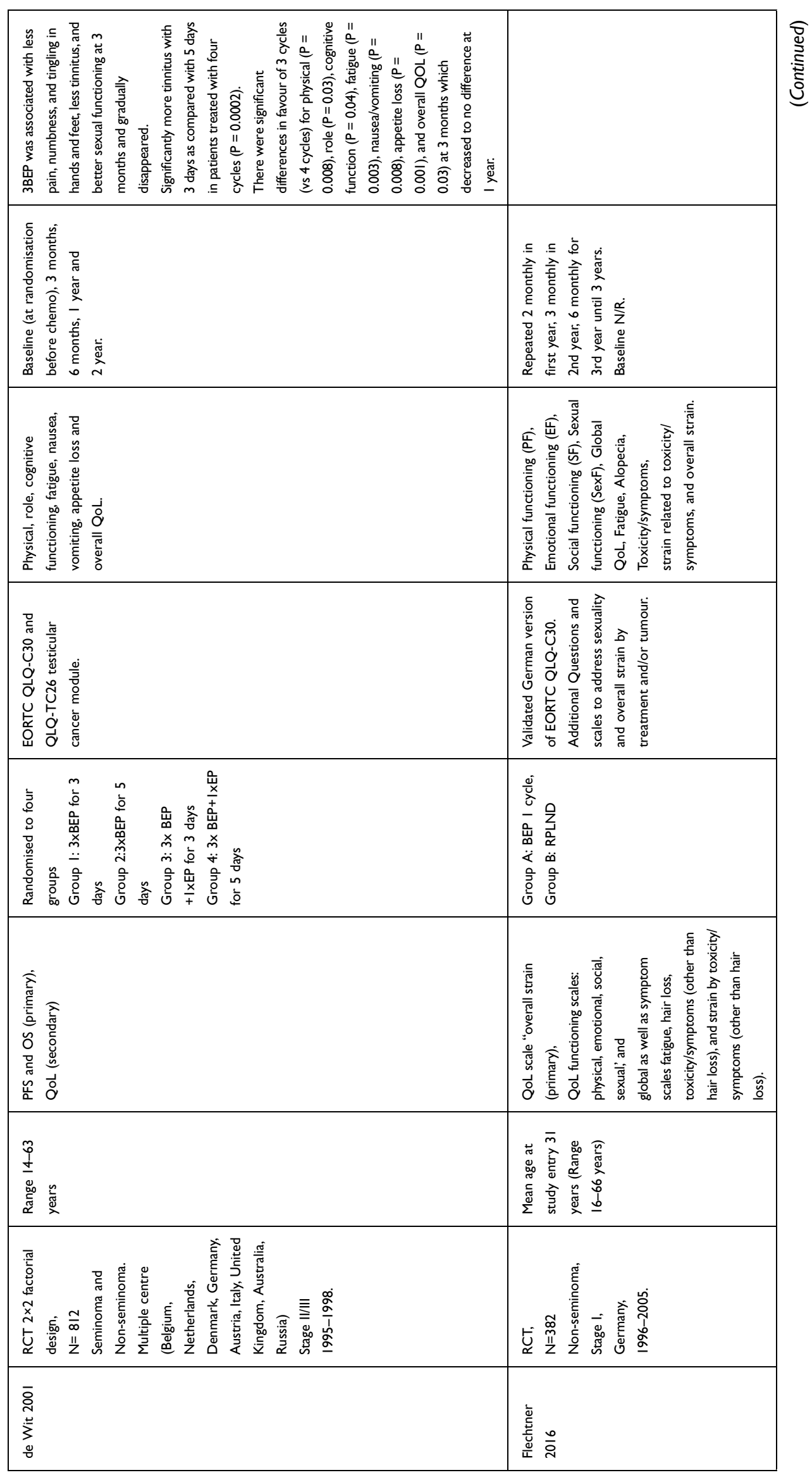




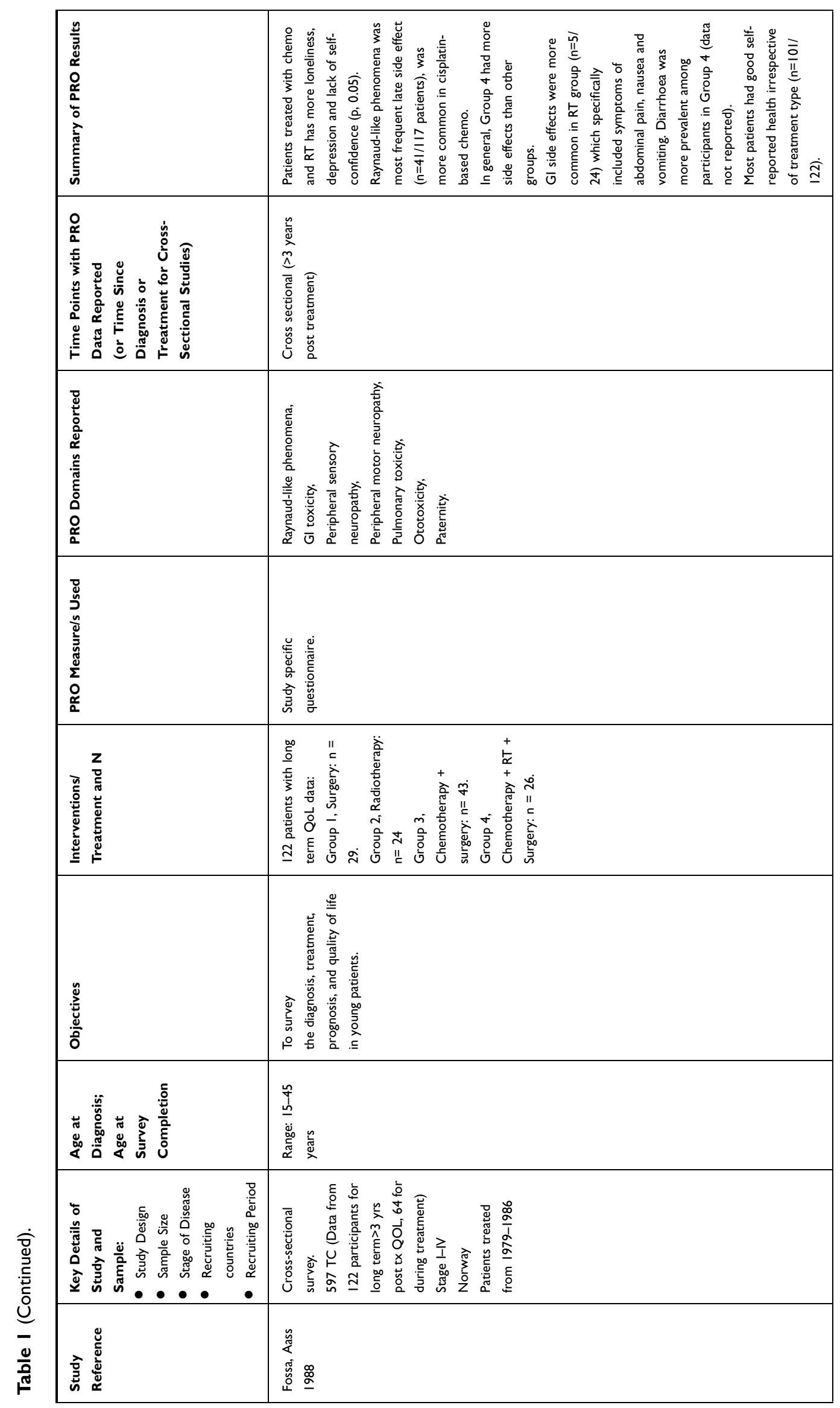




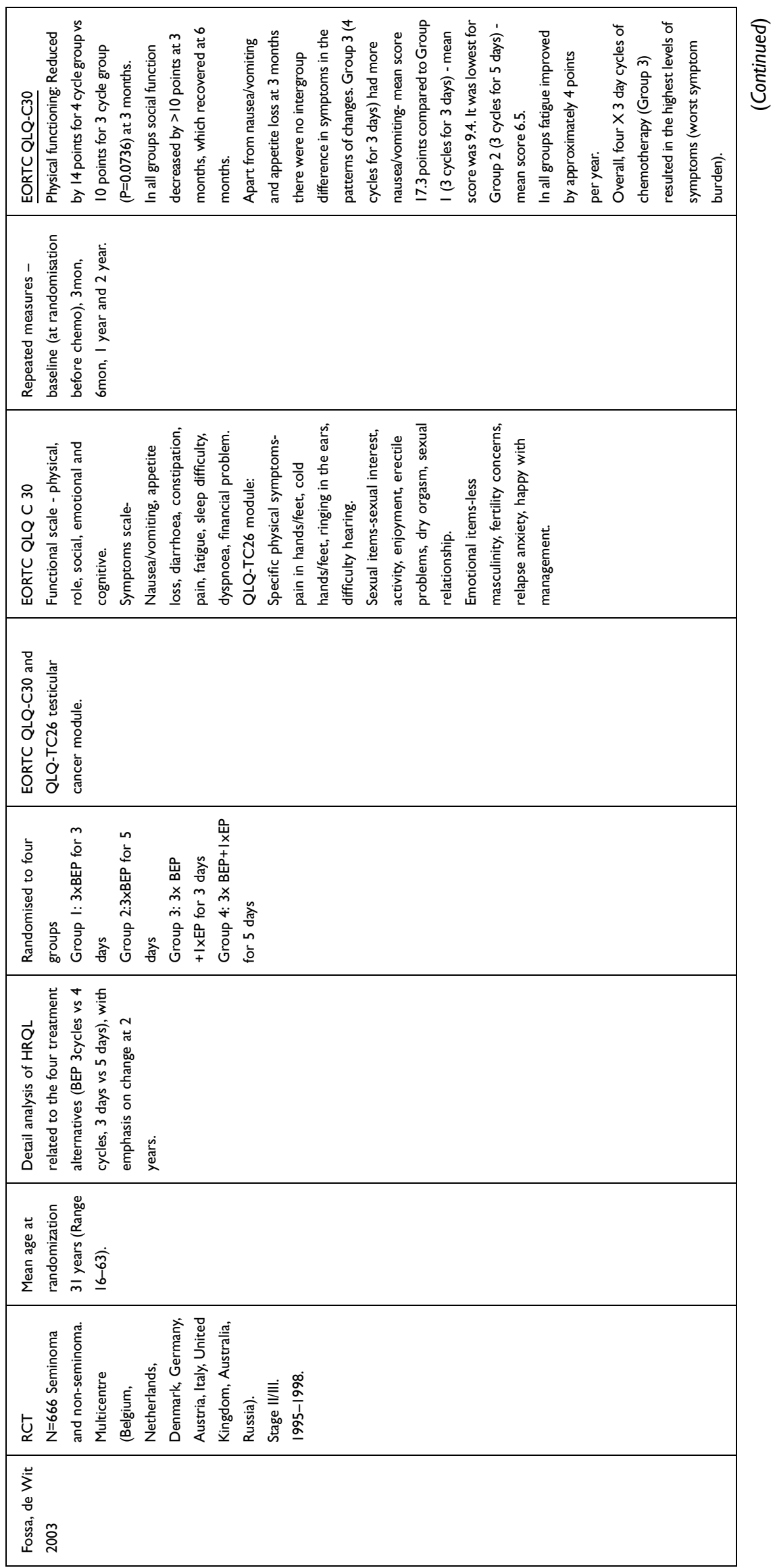




\begin{tabular}{|c|c|}
\hline 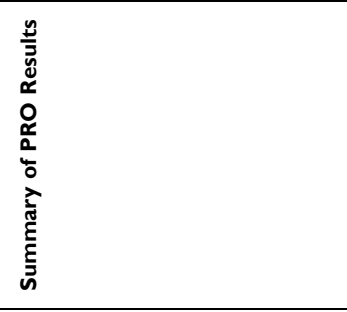 & 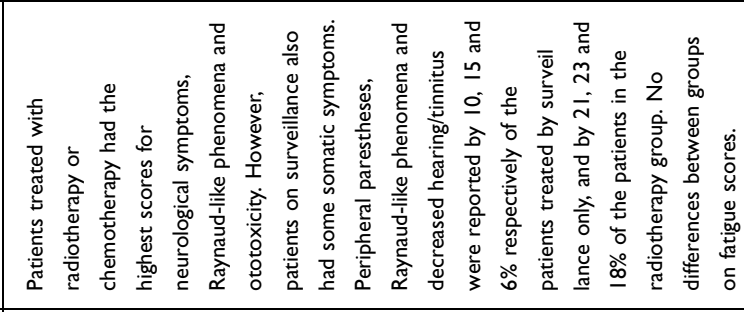 \\
\hline 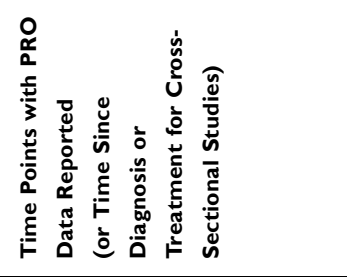 & 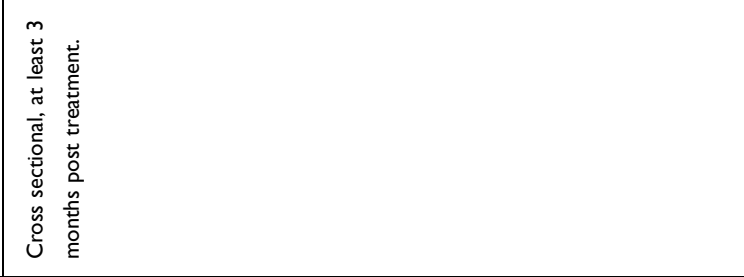 \\
\hline 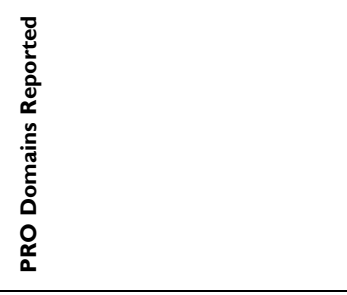 & 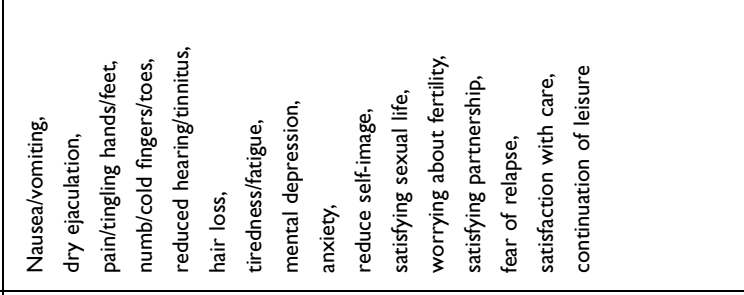 \\
\hline 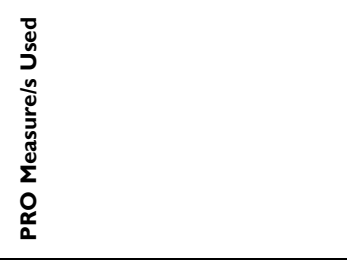 & 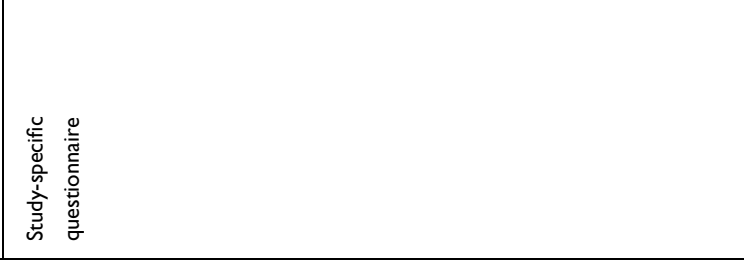 \\
\hline 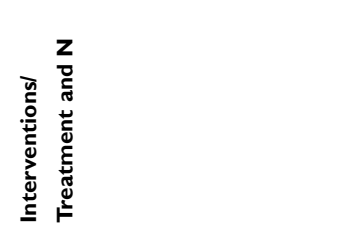 & 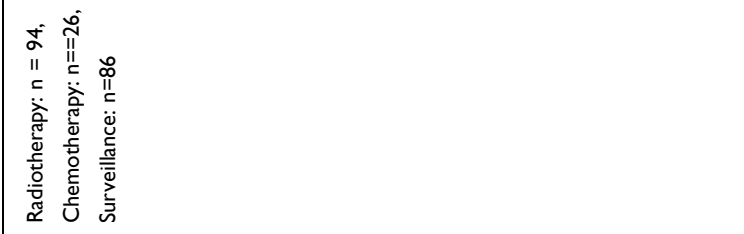 \\
\hline 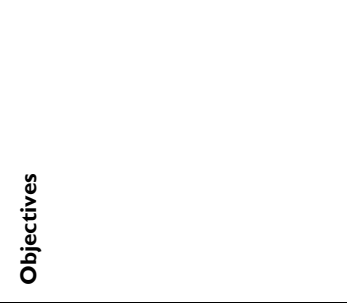 & 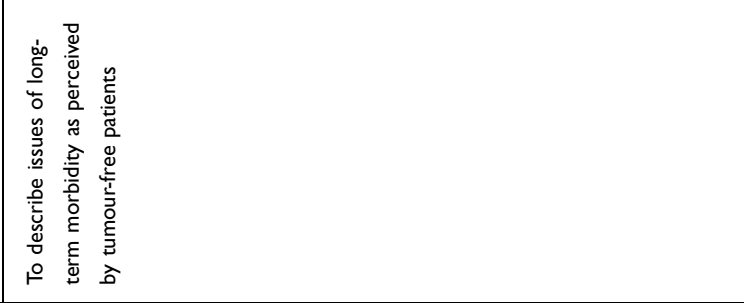 \\
\hline 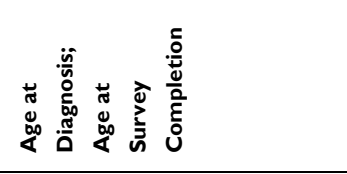 & 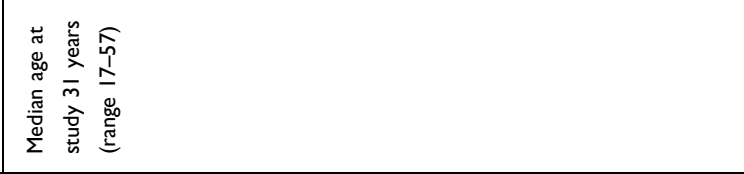 \\
\hline 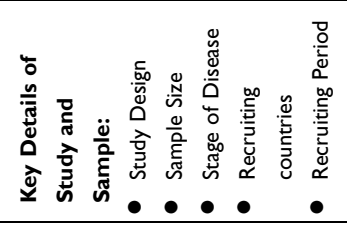 & 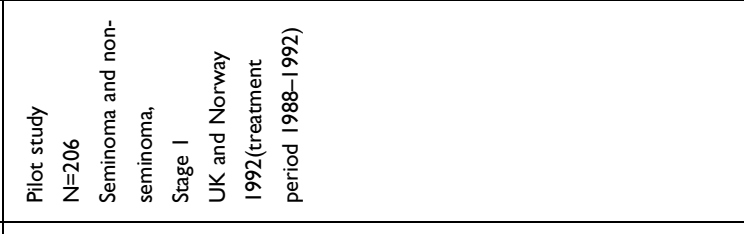 \\
\hline 旁彥产 & 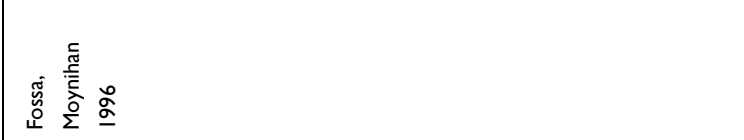 \\
\hline
\end{tabular}




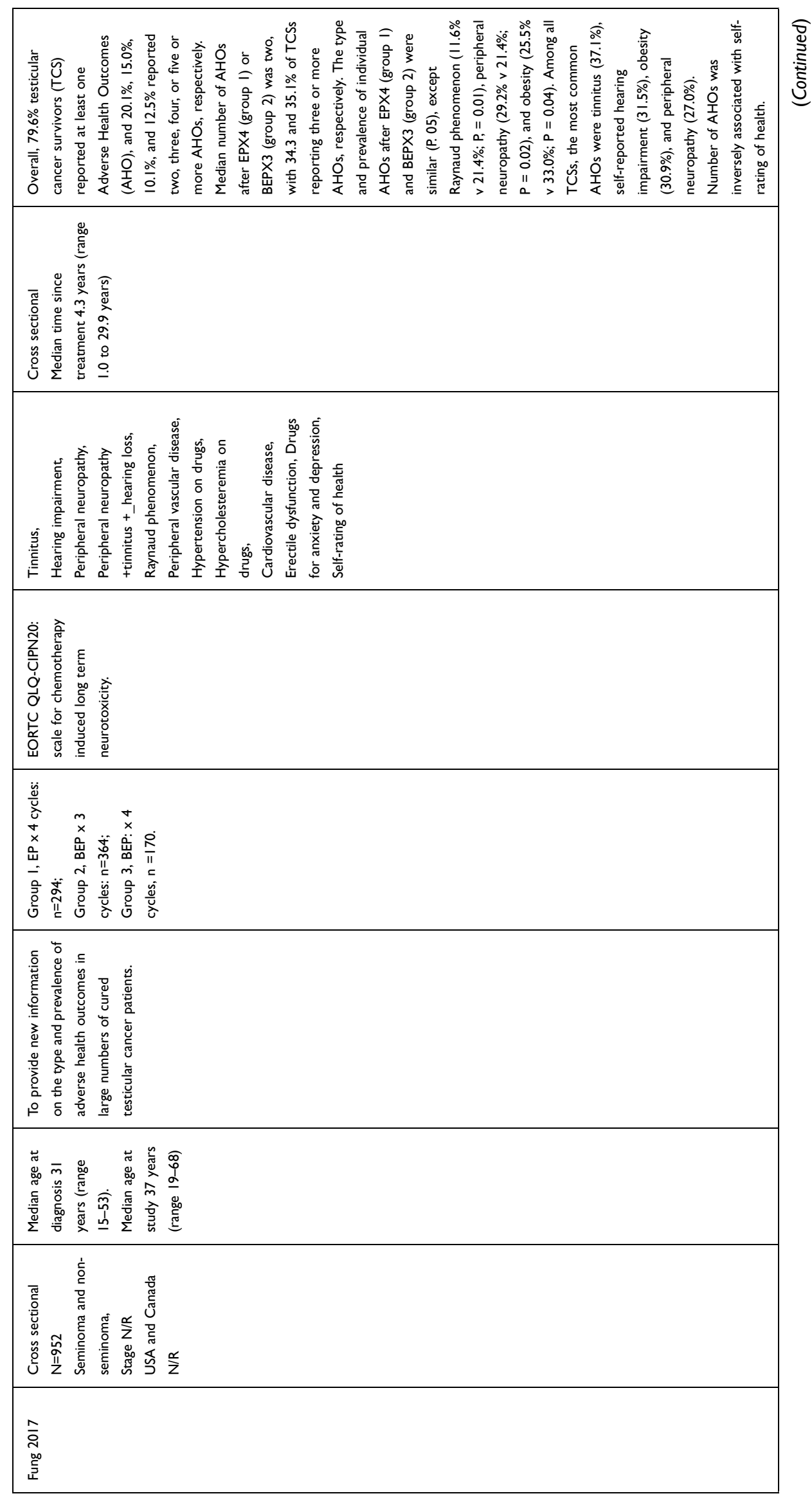




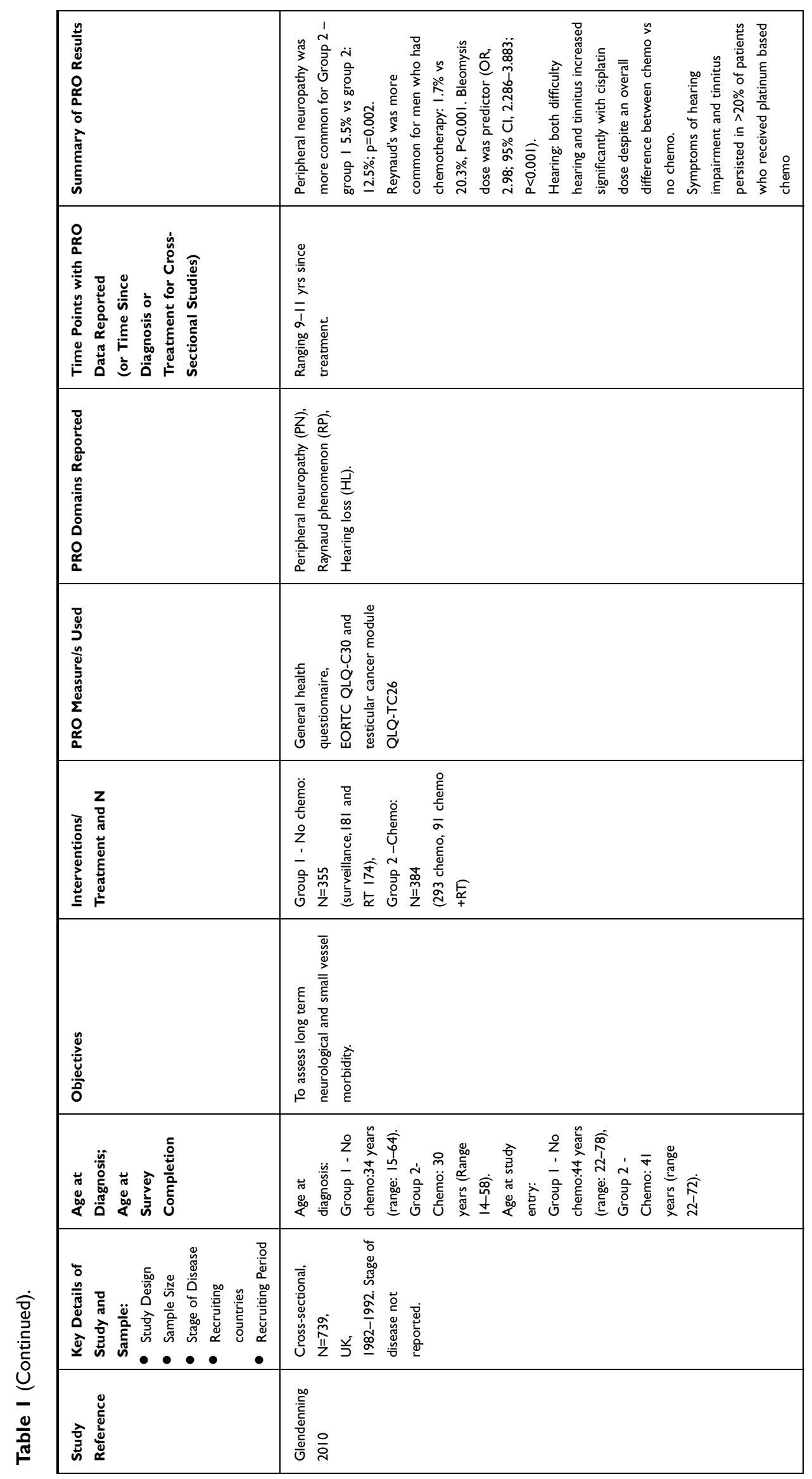




\begin{tabular}{|c|c|}
\hline 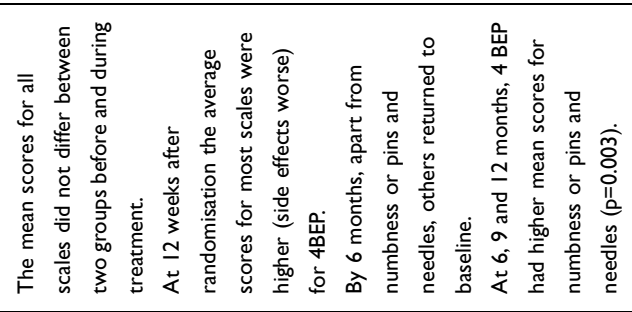 & 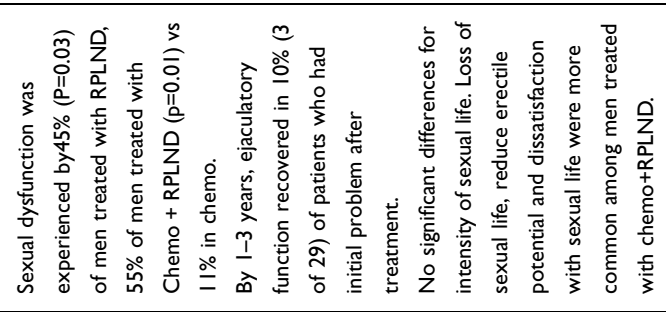 \\
\hline 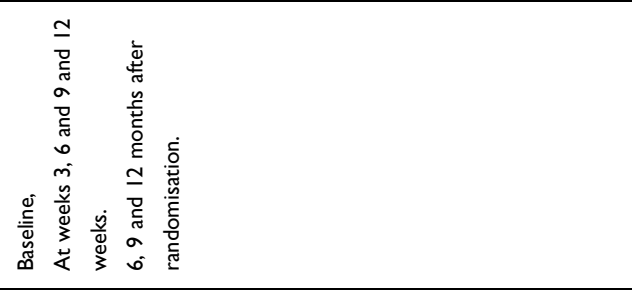 & 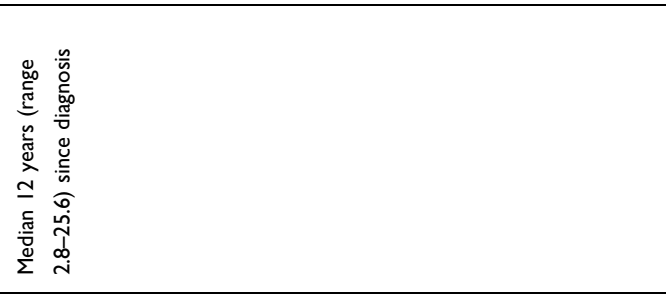 \\
\hline 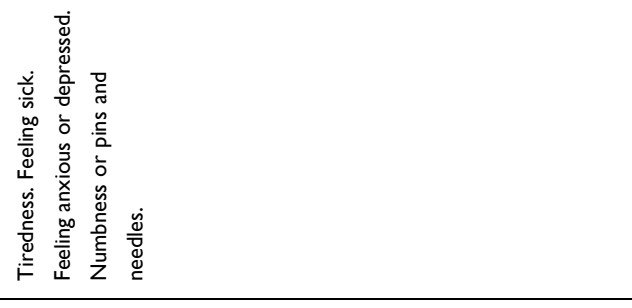 & 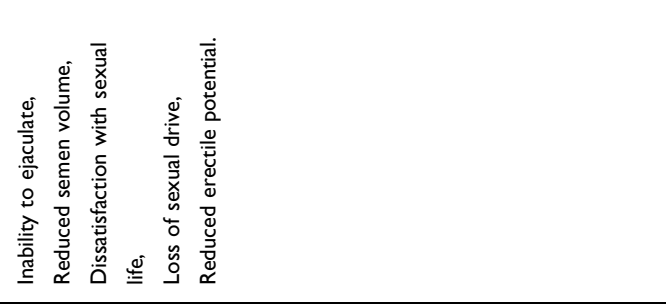 \\
\hline 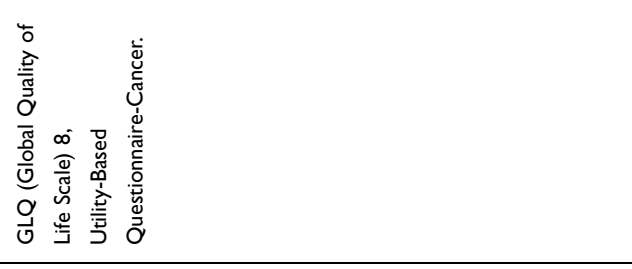 & 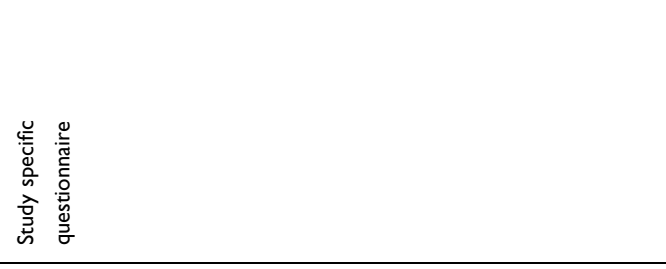 \\
\hline 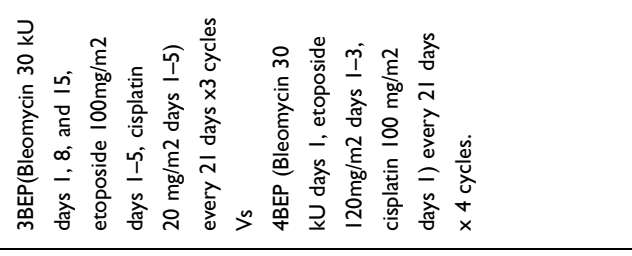 & 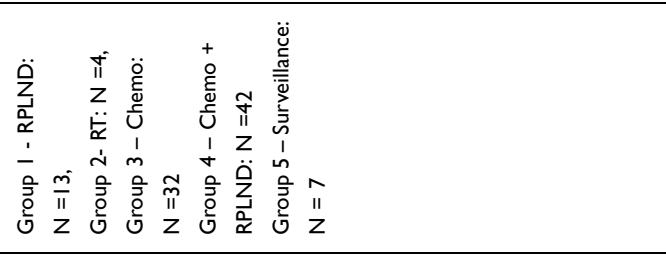 \\
\hline 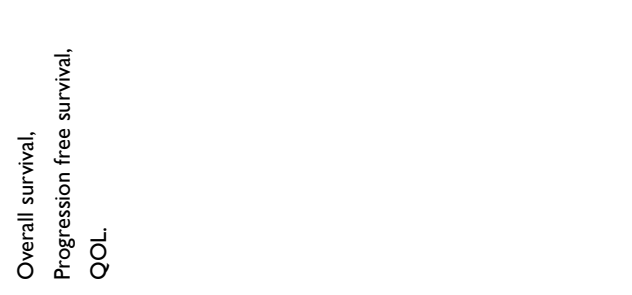 & 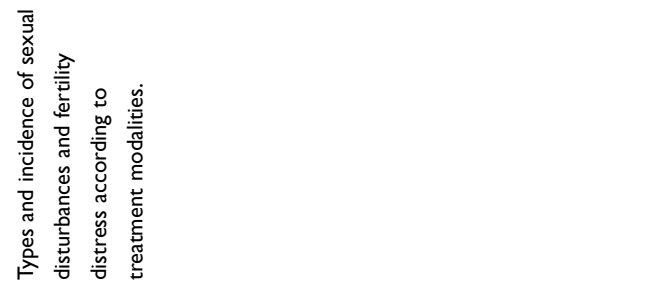 \\
\hline 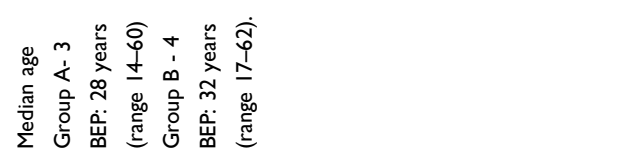 & 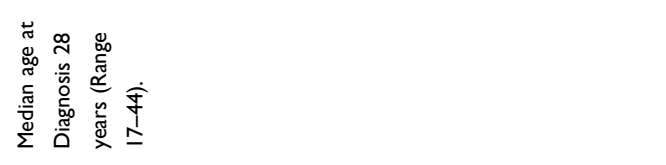 \\
\hline 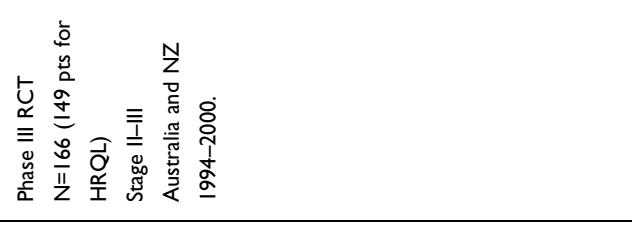 & 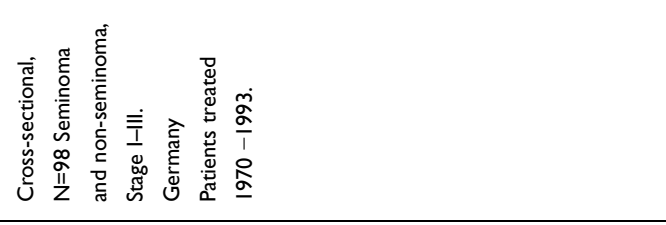 \\
\hline 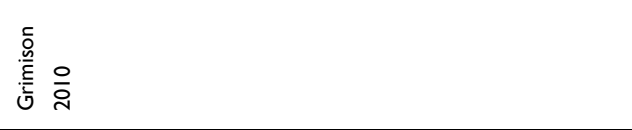 & 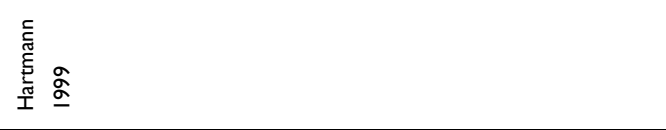 \\
\hline
\end{tabular}




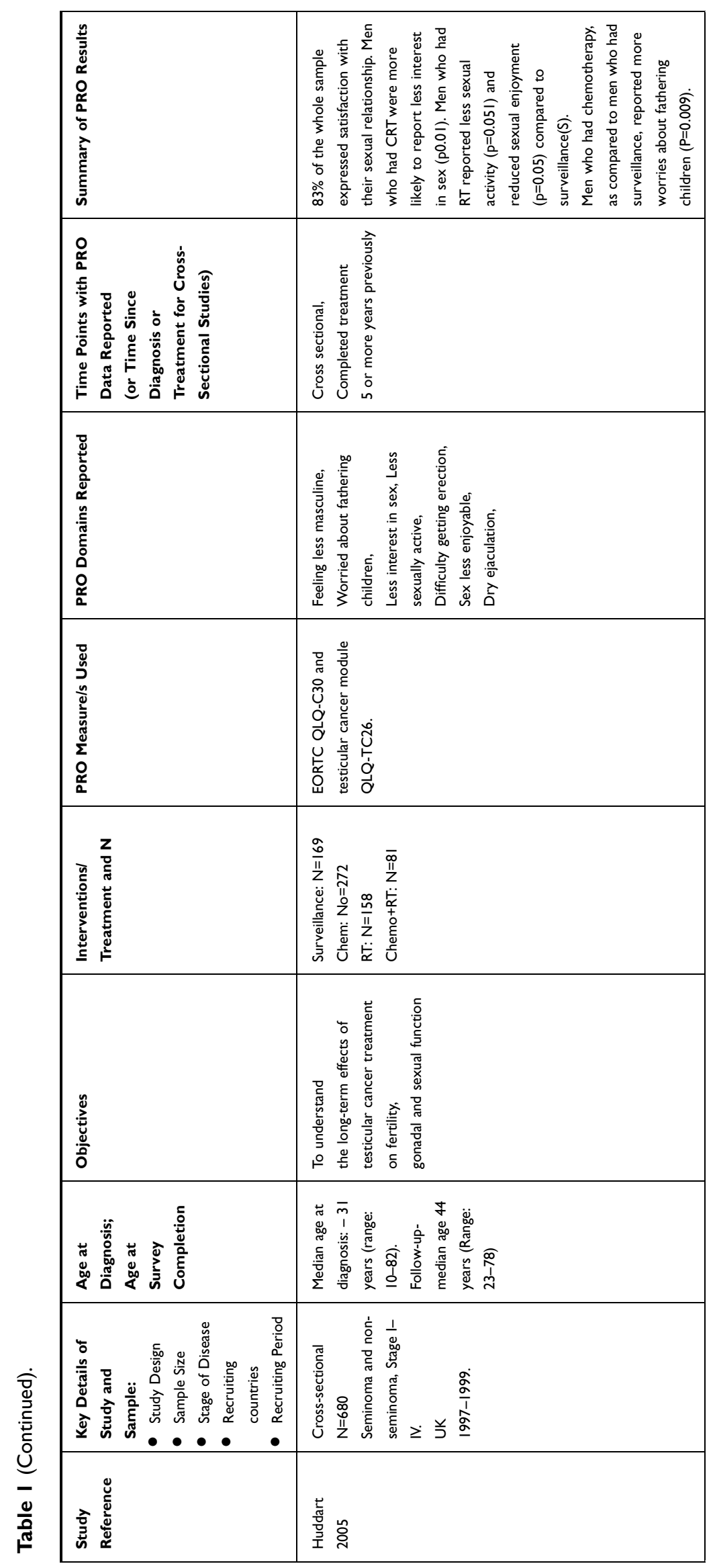




\begin{tabular}{|c|c|}
\hline 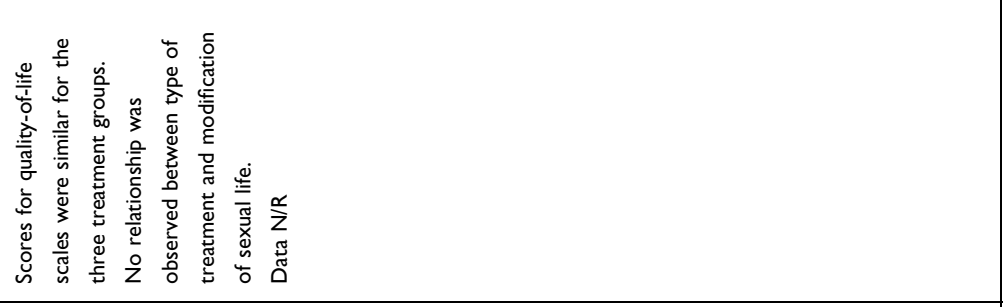 & 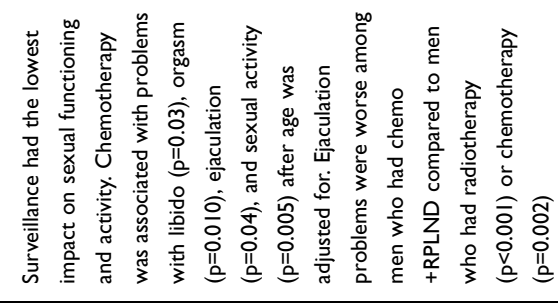 \\
\hline 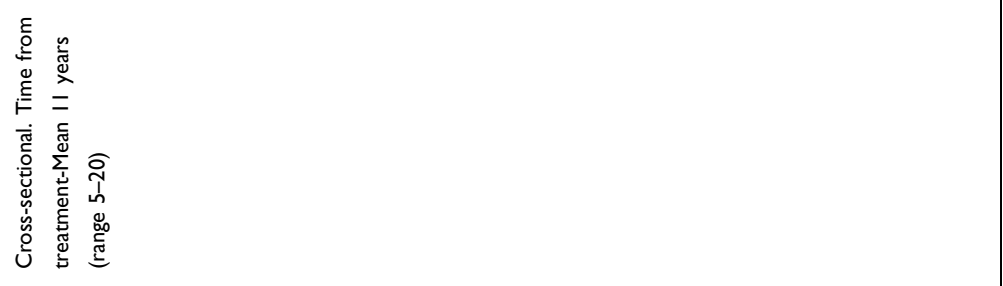 & 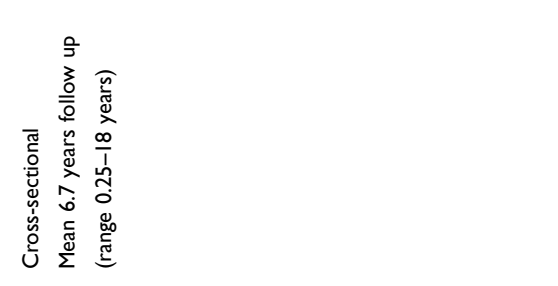 \\
\hline 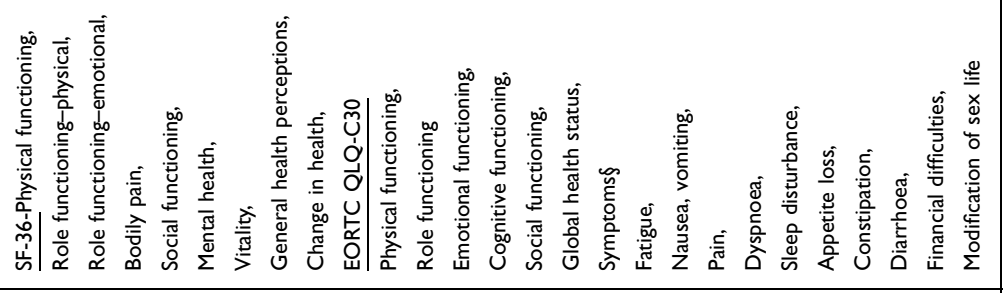 & 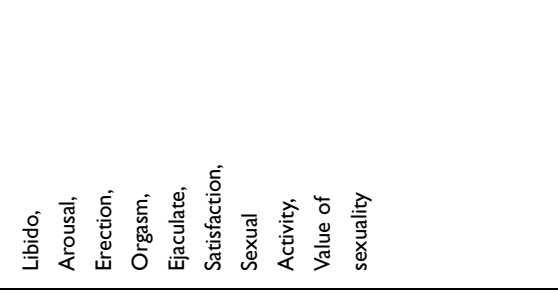 \\
\hline 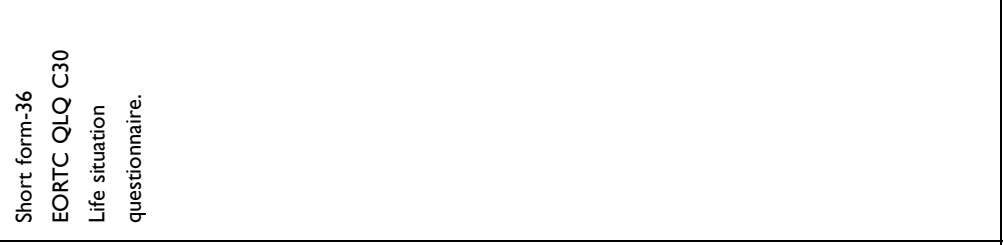 & 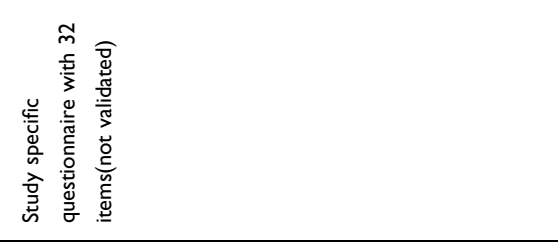 \\
\hline 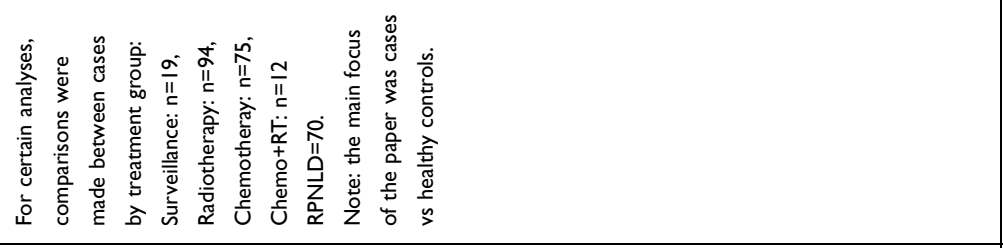 & 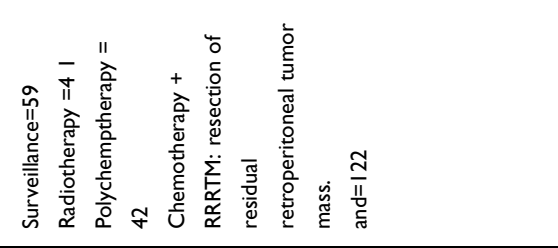 \\
\hline 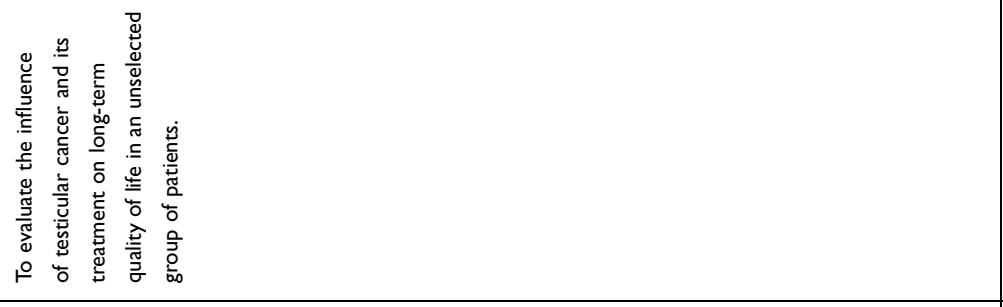 & 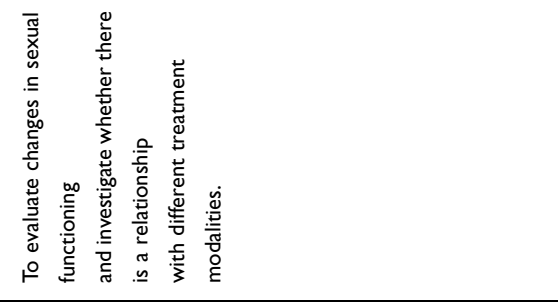 \\
\hline 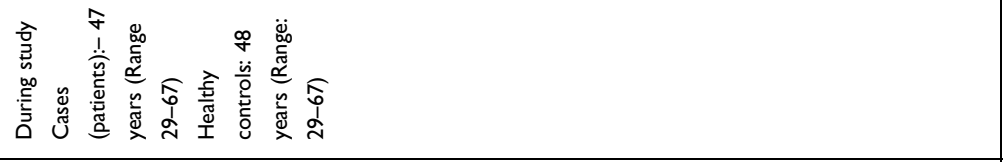 & 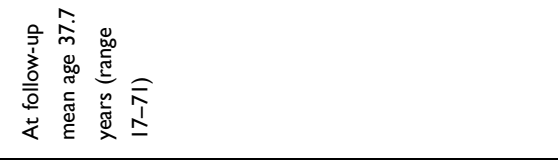 \\
\hline 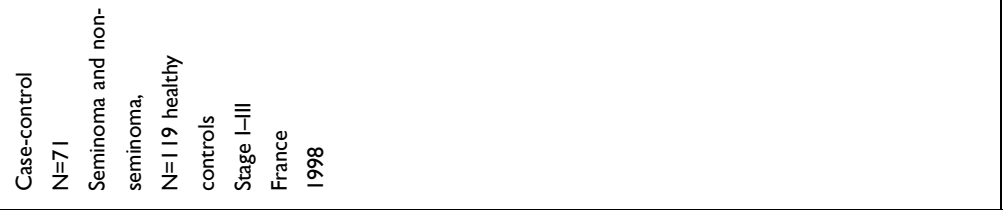 & 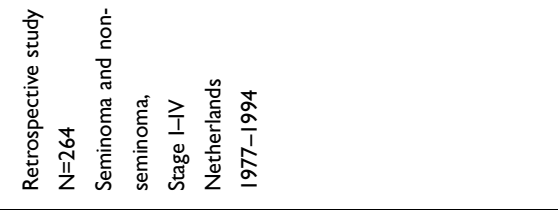 \\
\hline 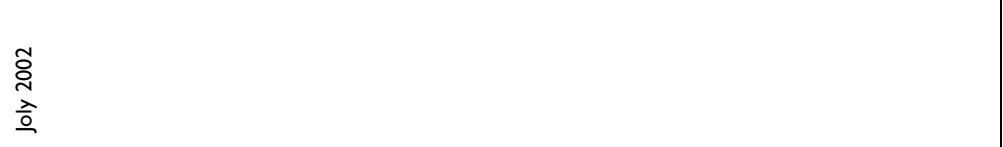 & 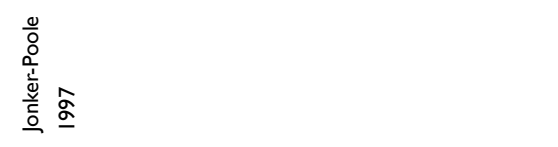 \\
\hline
\end{tabular}




\begin{tabular}{|c|c|}
\hline 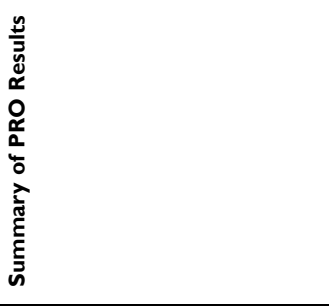 & 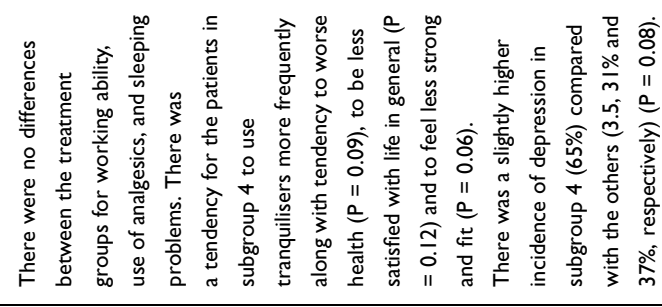 \\
\hline 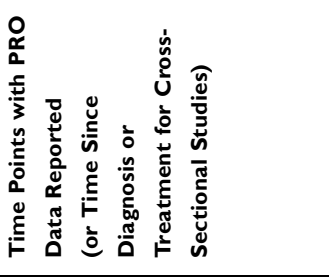 & 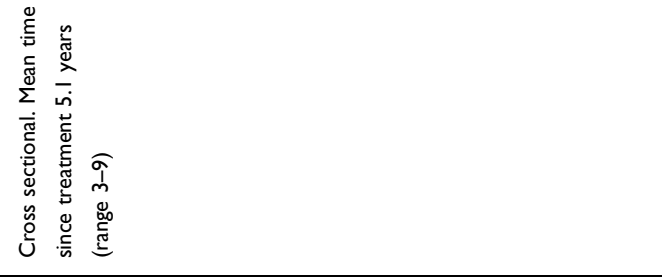 \\
\hline 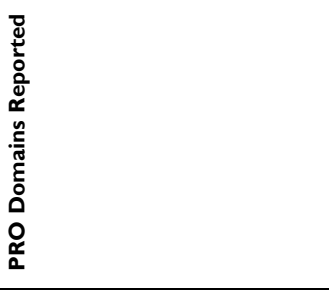 & 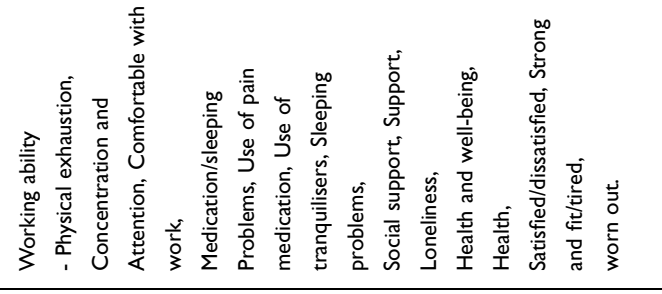 \\
\hline 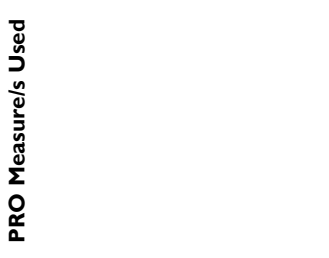 & 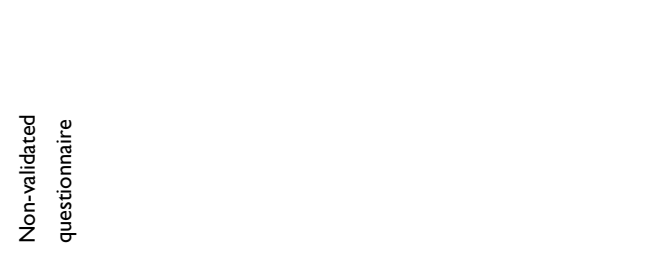 \\
\hline 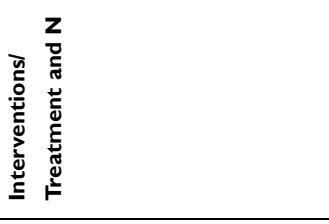 & 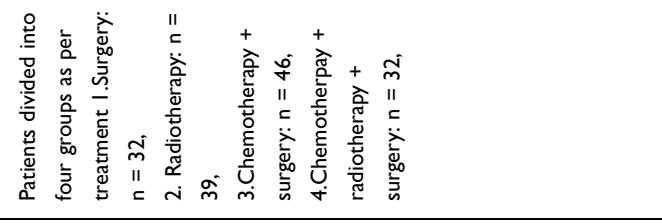 \\
\hline & 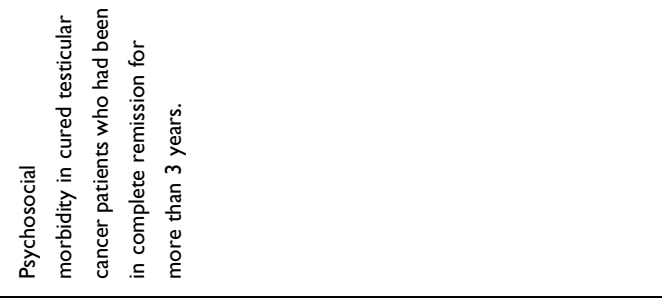 \\
\hline 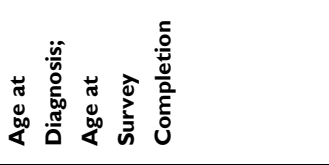 & 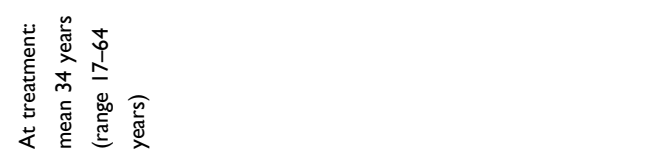 \\
\hline 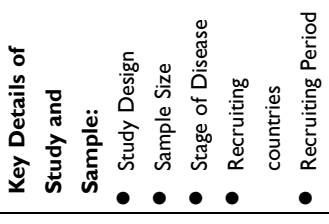 & 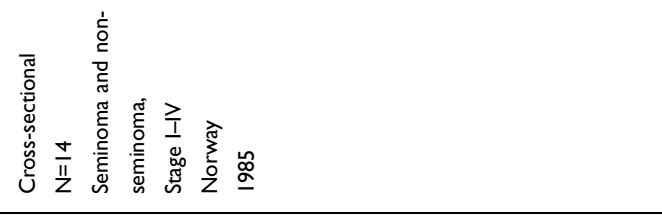 \\
\hline 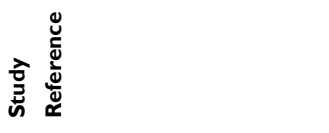 & $\begin{array}{l}\bar{\sigma} \\
\frac{\sigma}{\widetilde{g}} \\
\stackrel{\tilde{g}}{凶} \\
\end{array}$ \\
\hline
\end{tabular}




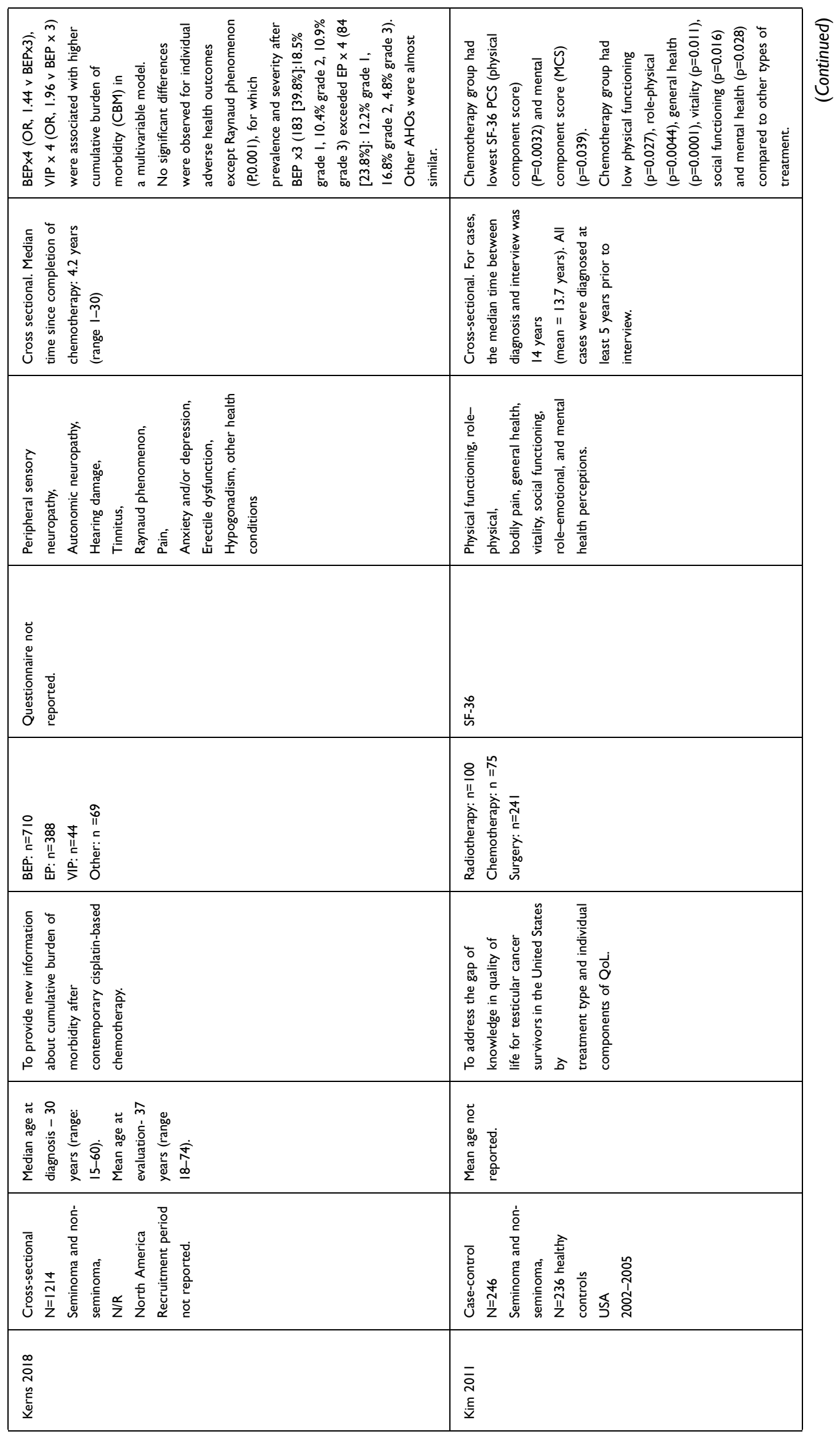




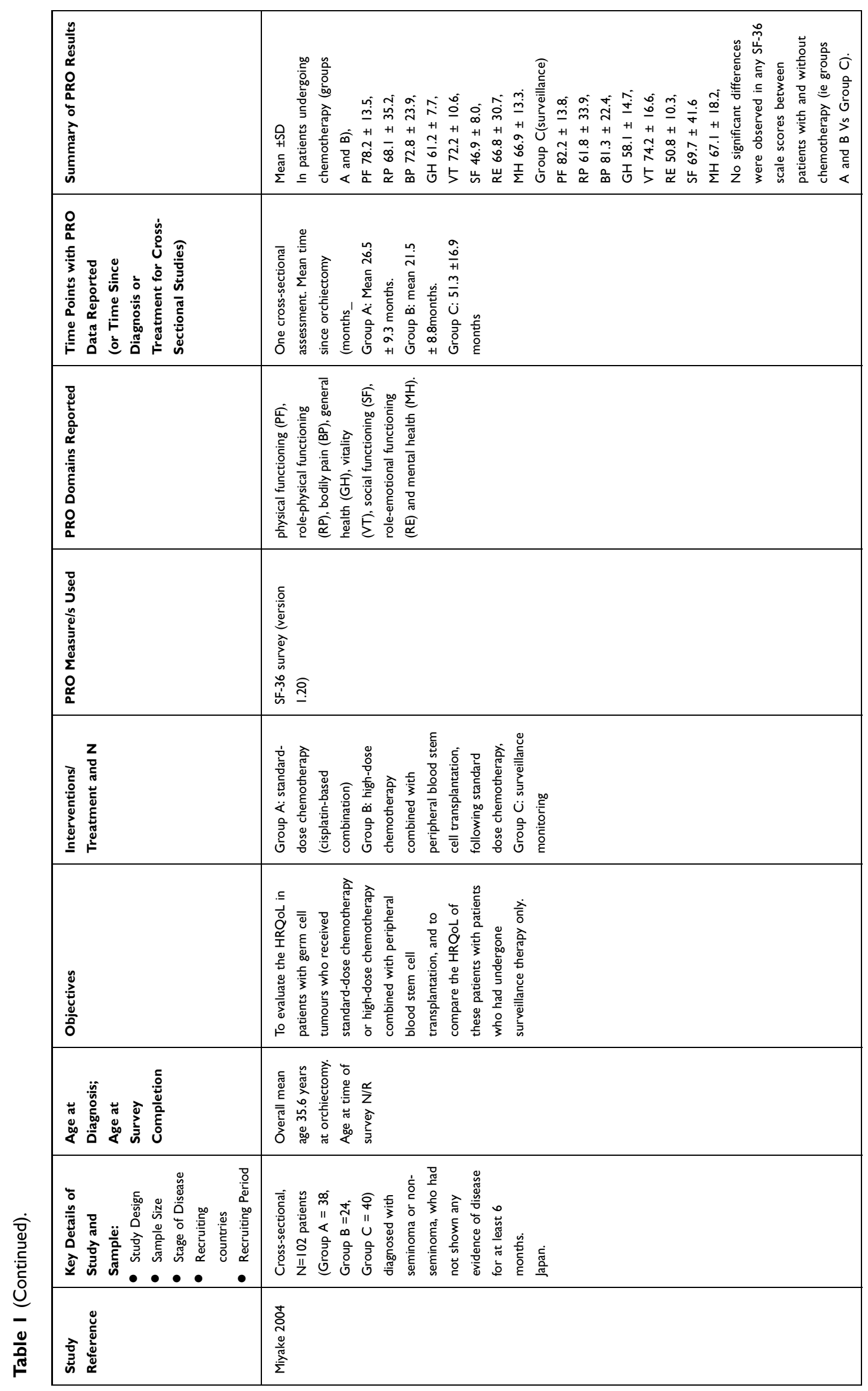




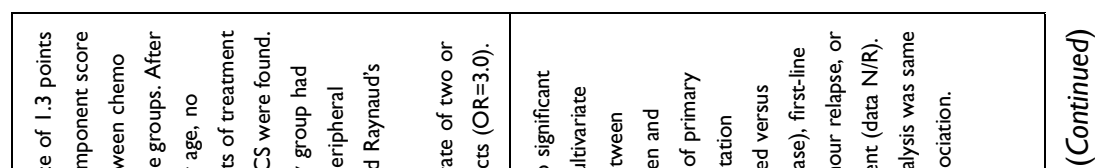

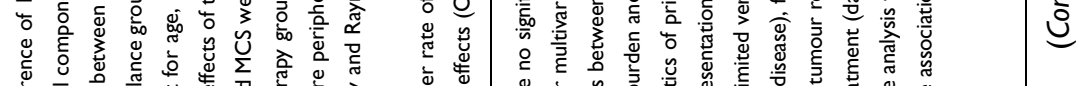

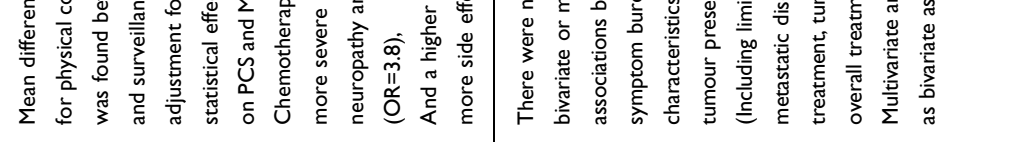

\begin{tabular}{|c|c|}
\hline 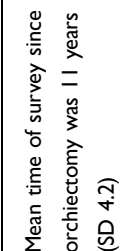 & 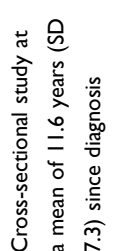 \\
\hline
\end{tabular}

\begin{tabular}{|c|c|}
\hline 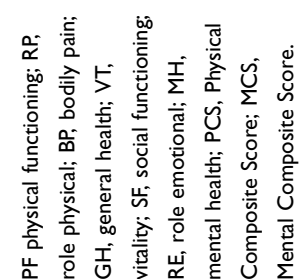 & 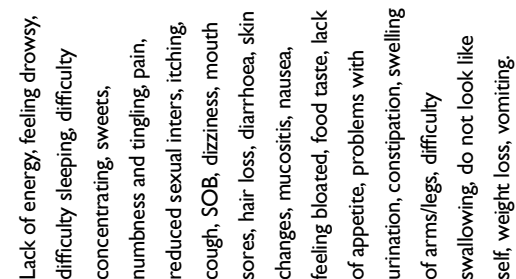 \\
\hline
\end{tabular}

\begin{tabular}{|c|c|}
\hline 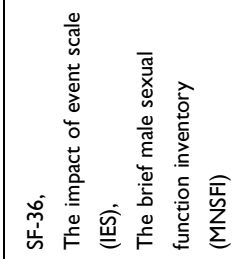 & 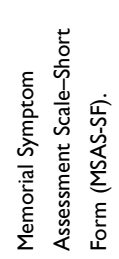 \\
\hline
\end{tabular}

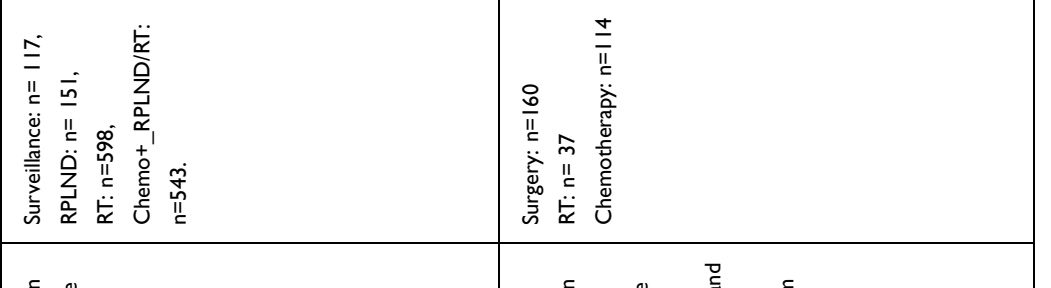

\begin{tabular}{|c|c|}
\hline 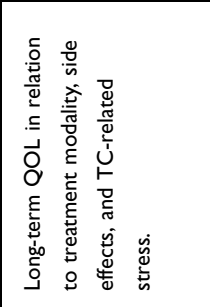 & 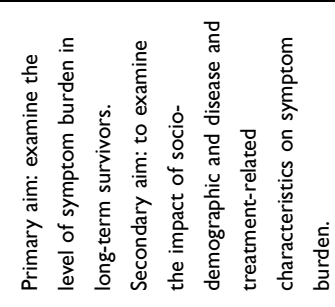 \\
\hline 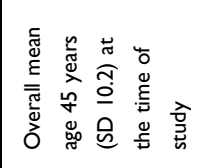 & 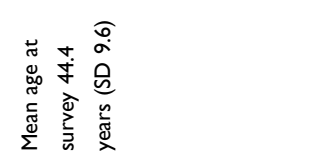 \\
\hline 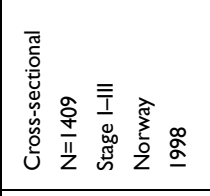 & 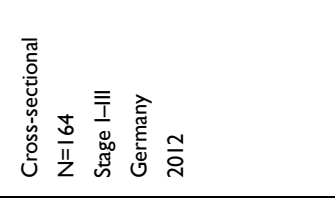 \\
\hline 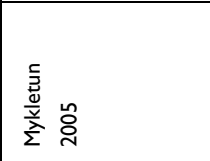 & 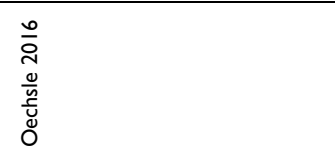 \\
\hline
\end{tabular}




\begin{tabular}{|c|c|}
\hline 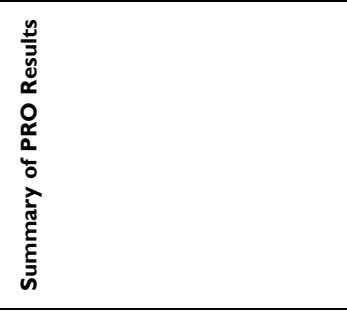 & 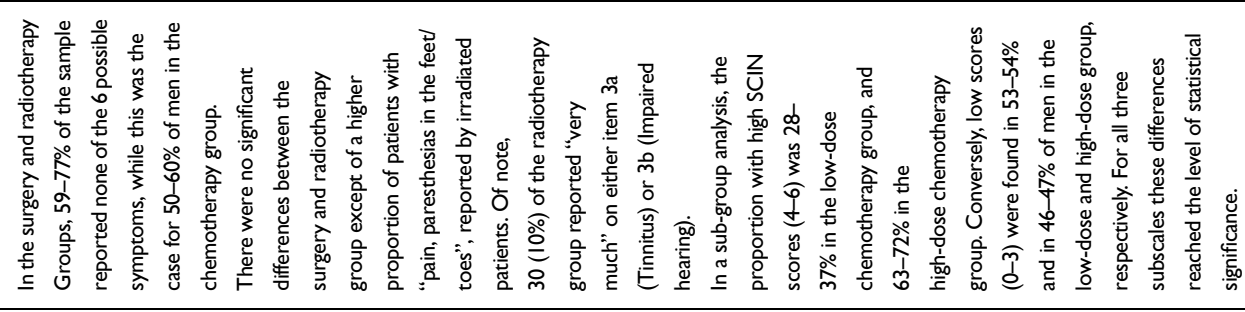 \\
\hline 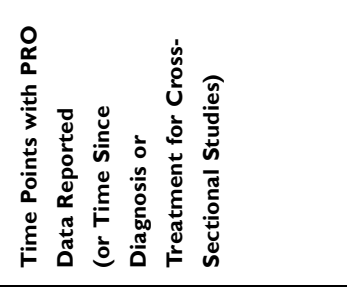 & 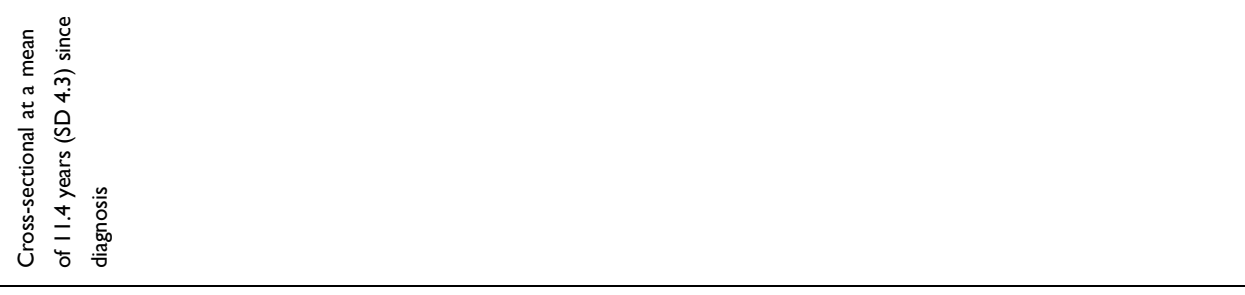 \\
\hline 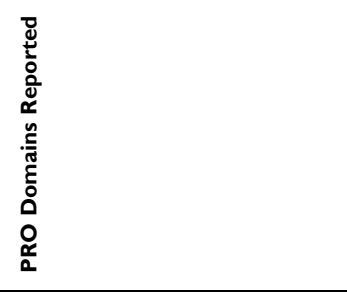 & 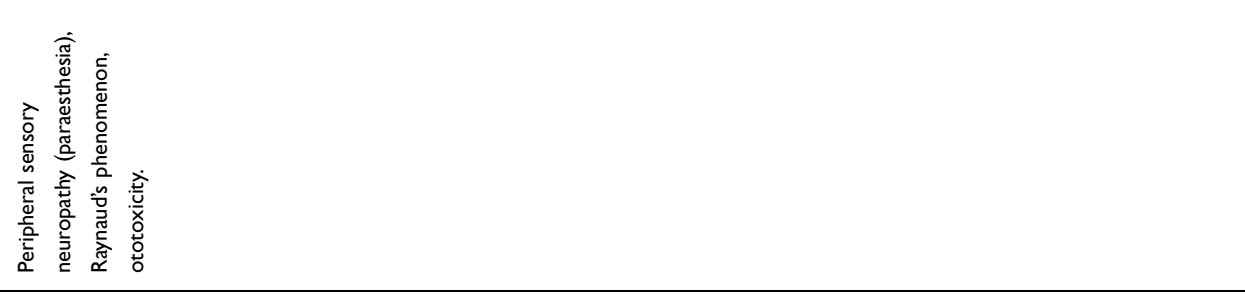 \\
\hline 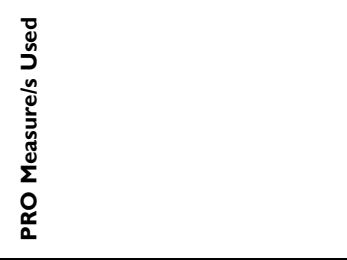 & 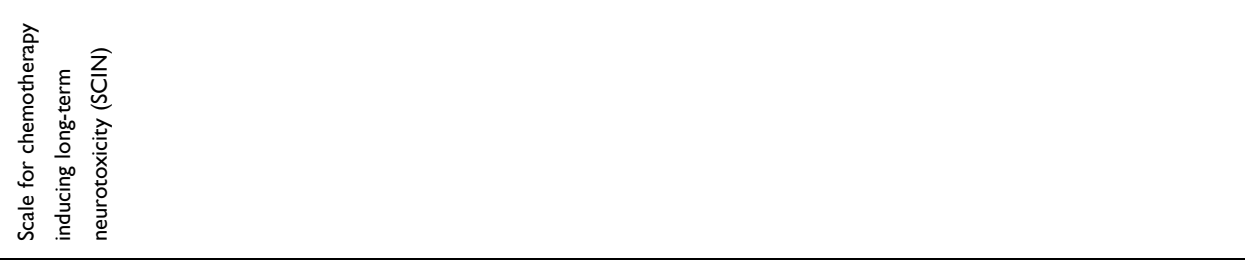 \\
\hline 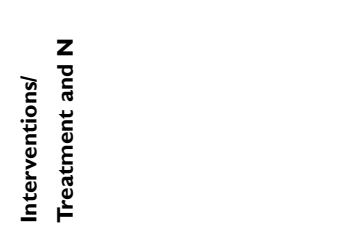 & 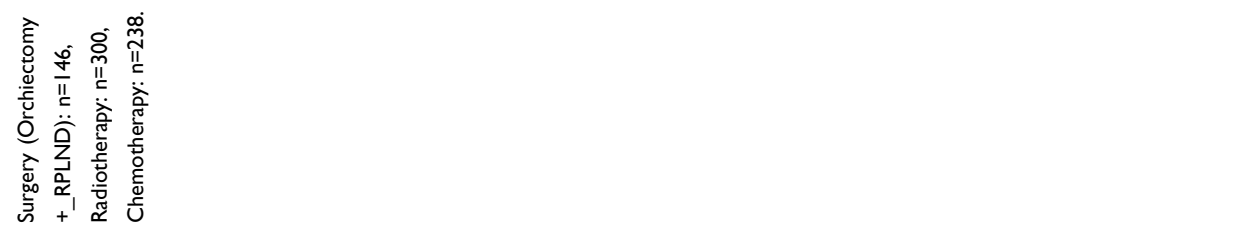 \\
\hline 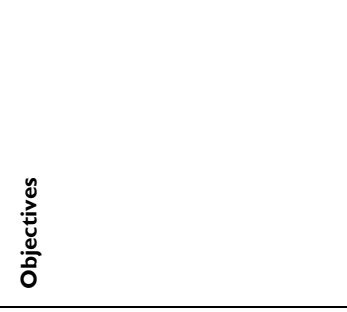 & 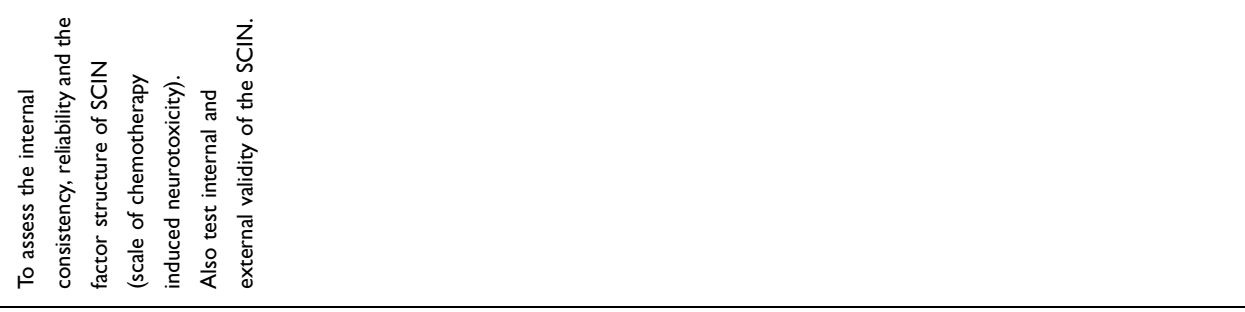 \\
\hline 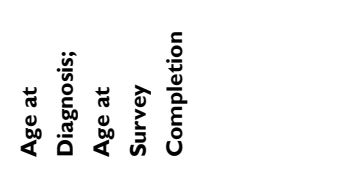 & 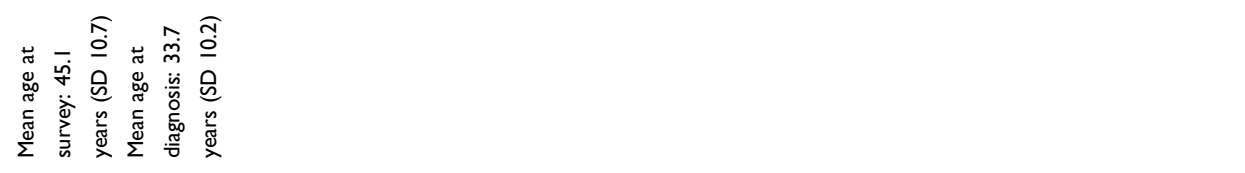 \\
\hline 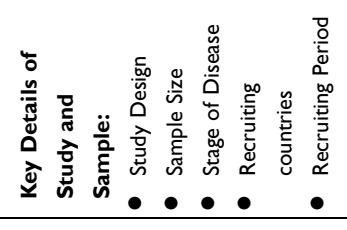 & 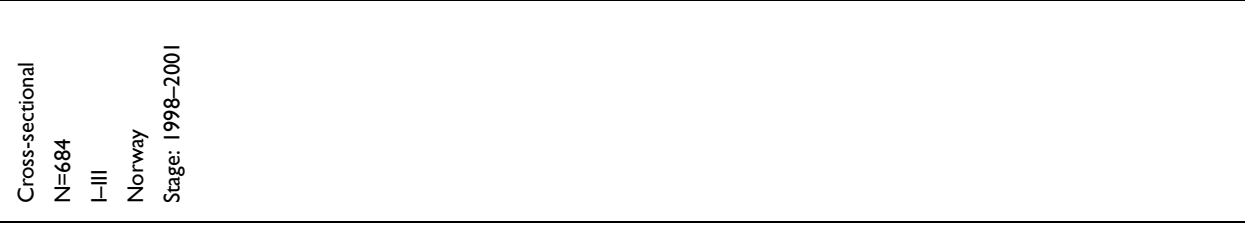 \\
\hline 总 & 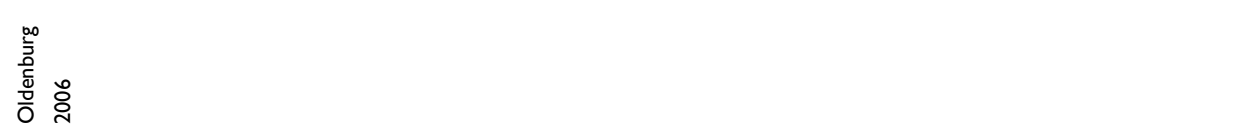 \\
\hline
\end{tabular}




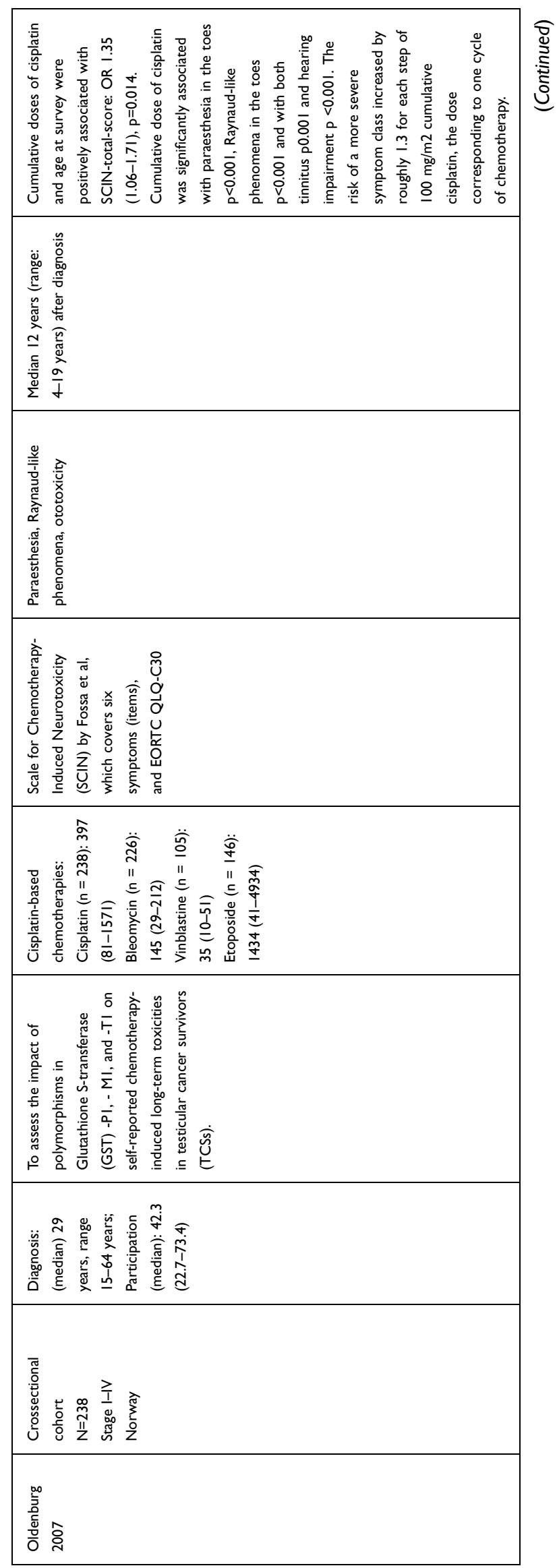




\begin{tabular}{|c|c|}
\hline & 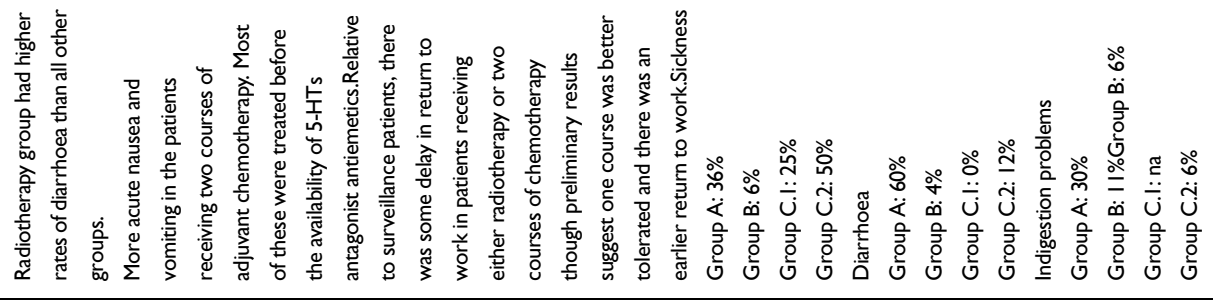 \\
\hline 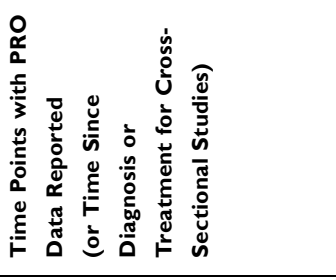 & 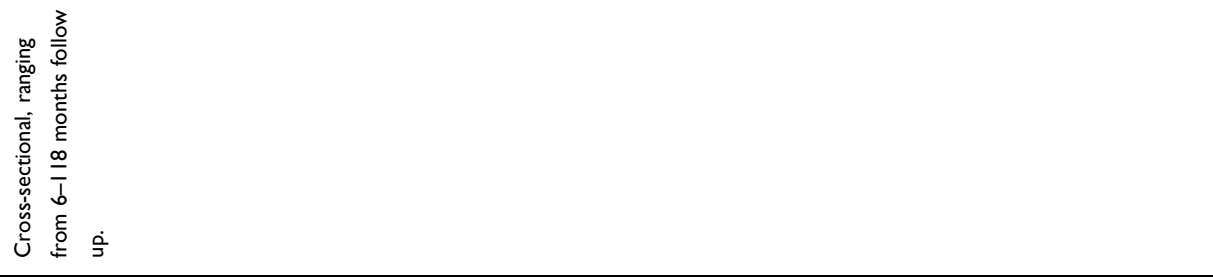 \\
\hline 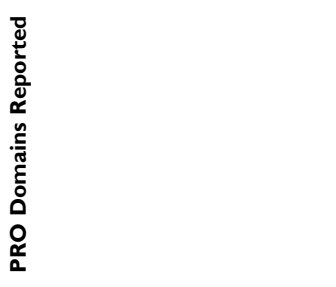 & 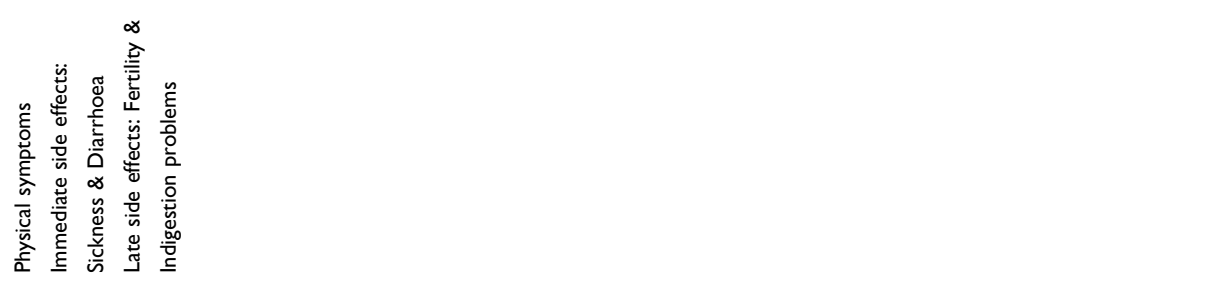 \\
\hline & \\
\hline 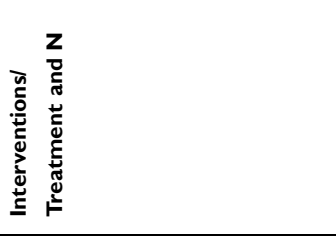 & 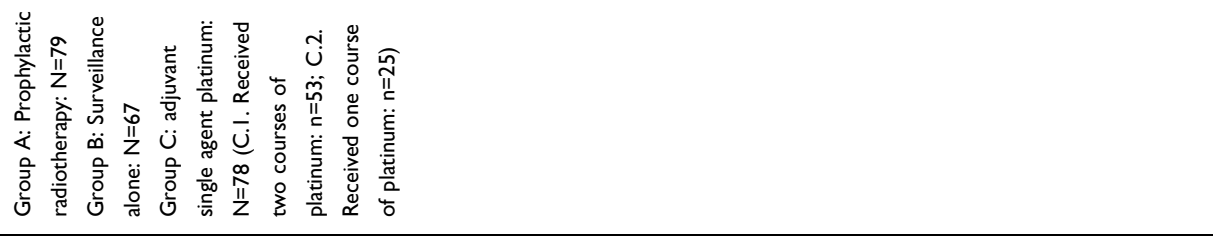 \\
\hline & 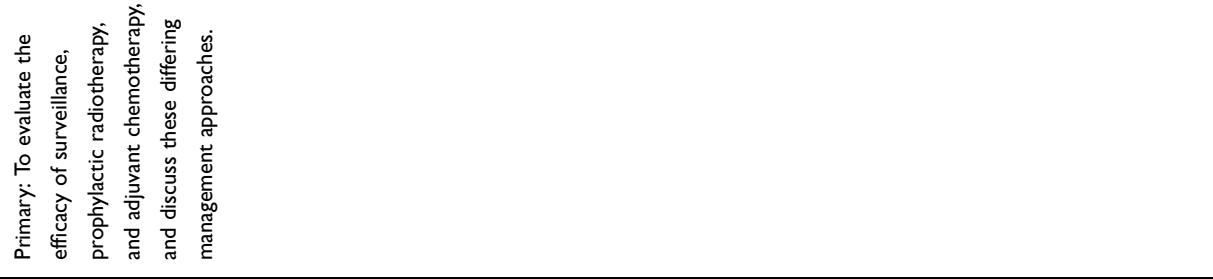 \\
\hline 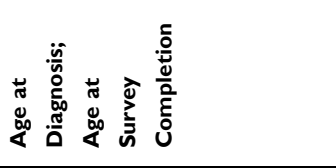 & 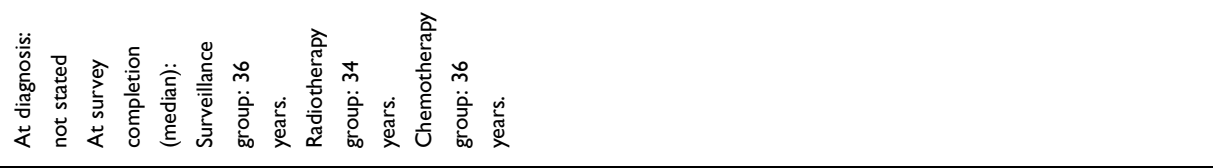 \\
\hline 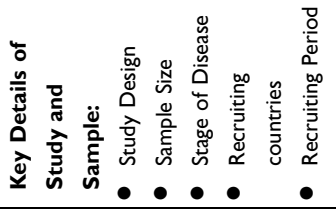 & 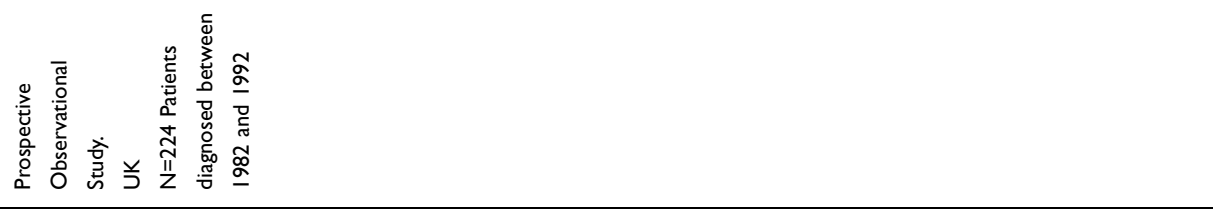 \\
\hline 密 & 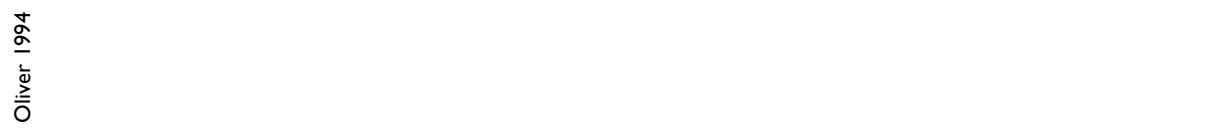 \\
\hline
\end{tabular}




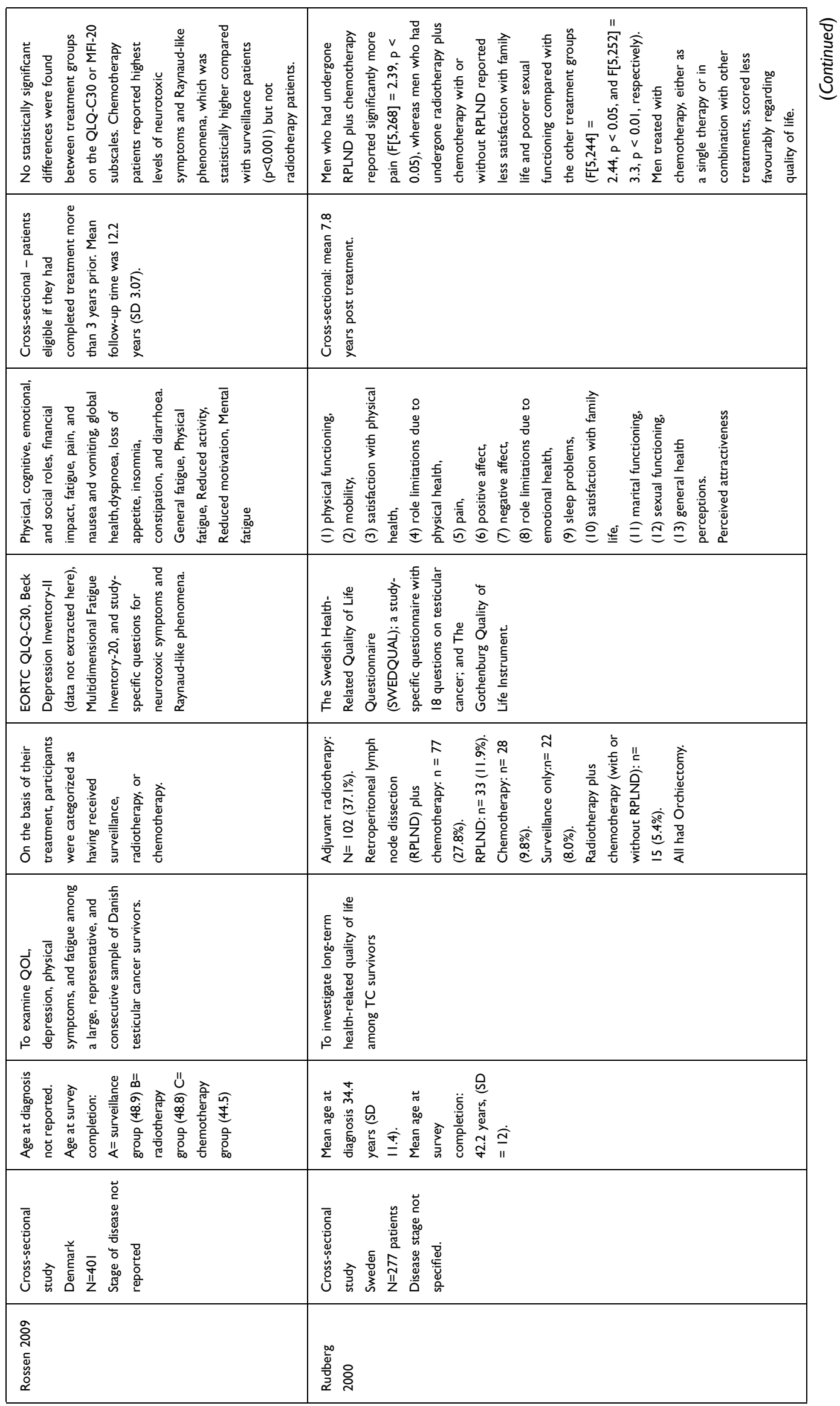




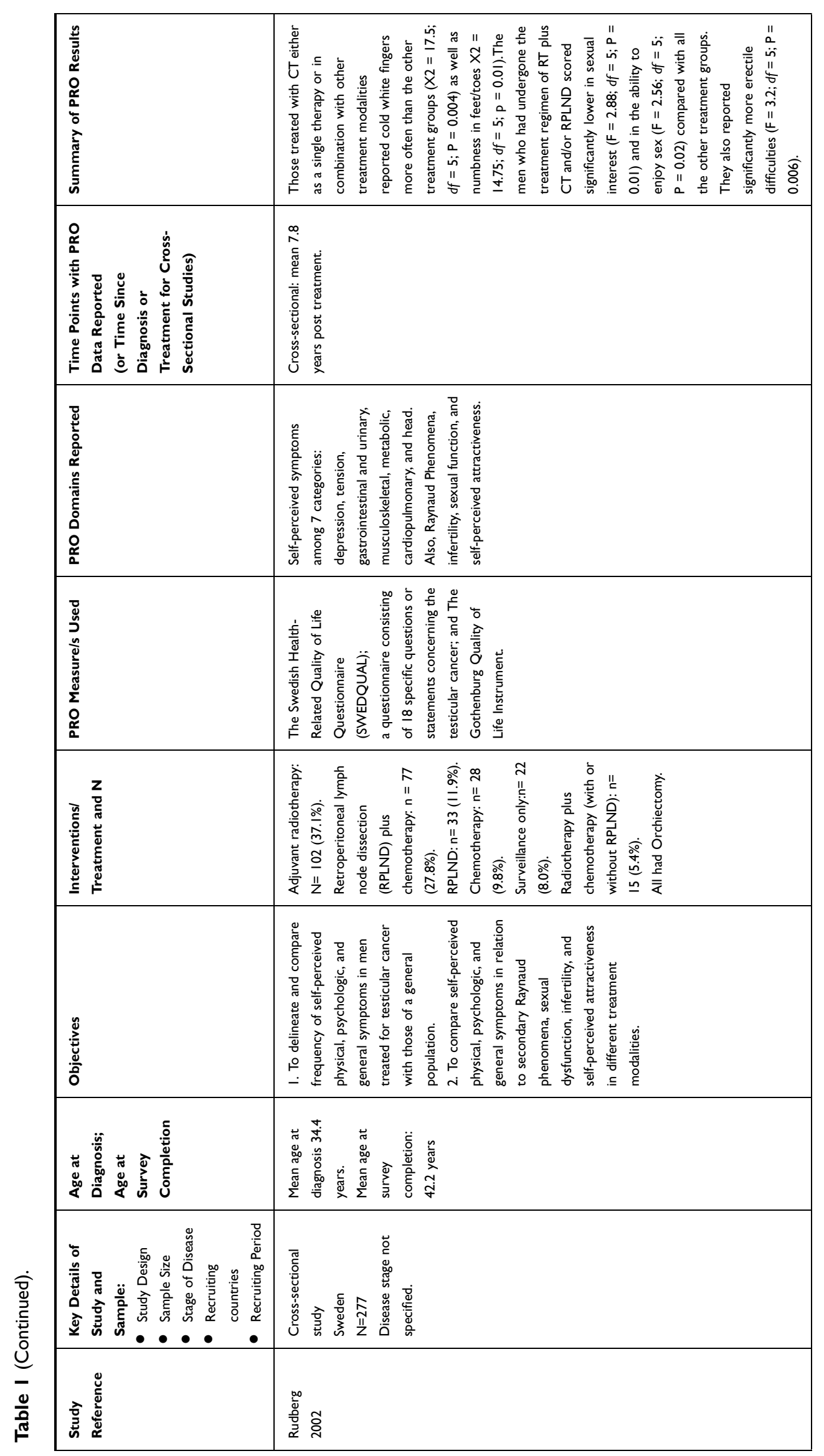




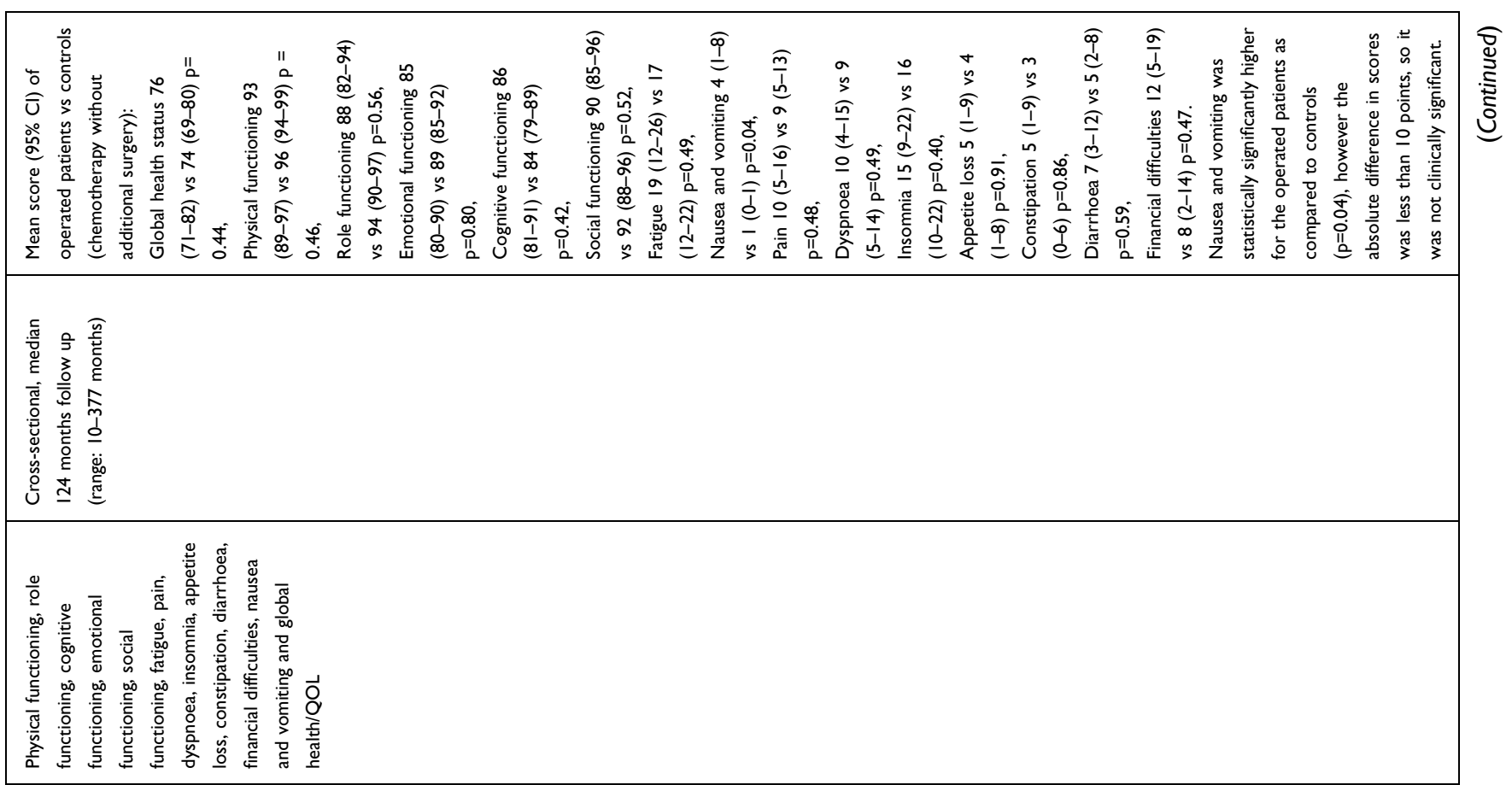

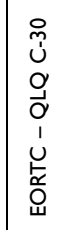
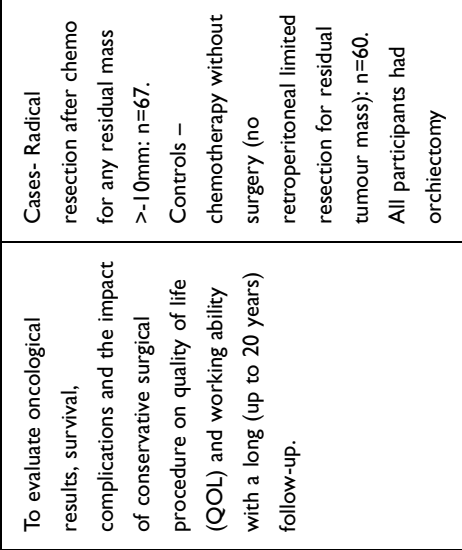

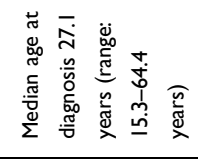

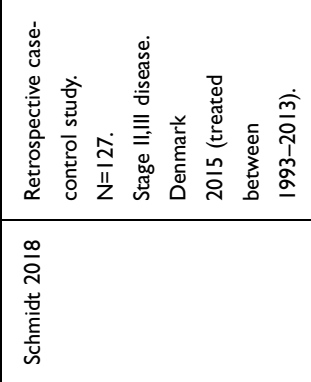




\begin{tabular}{|c|c|}
\hline 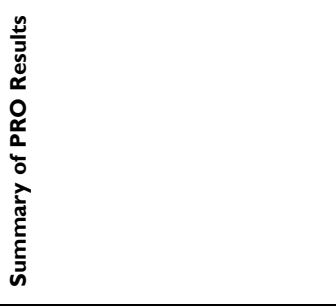 & 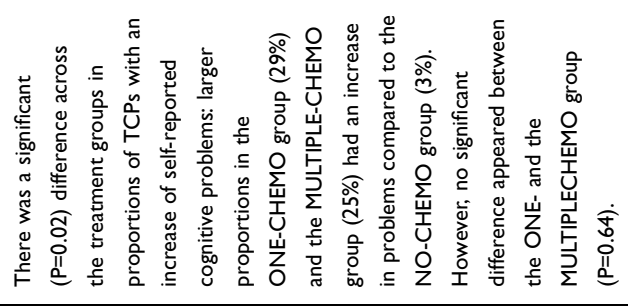 \\
\hline 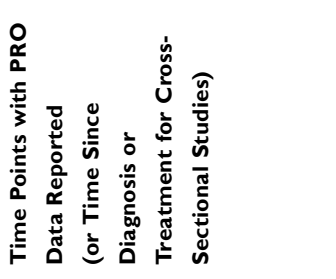 & 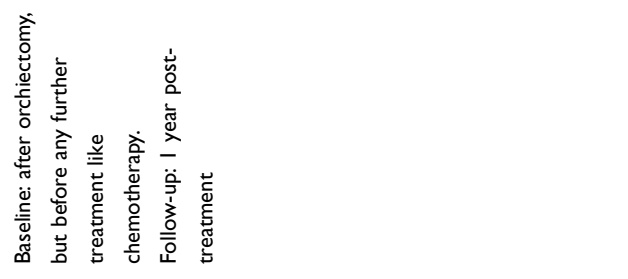 \\
\hline 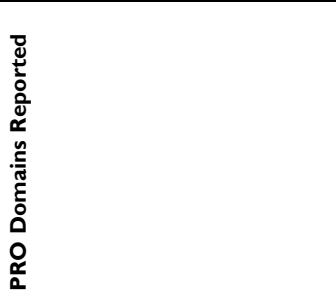 & 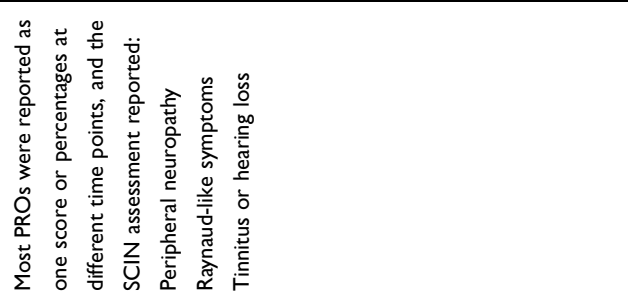 \\
\hline 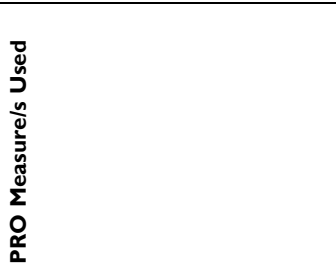 & 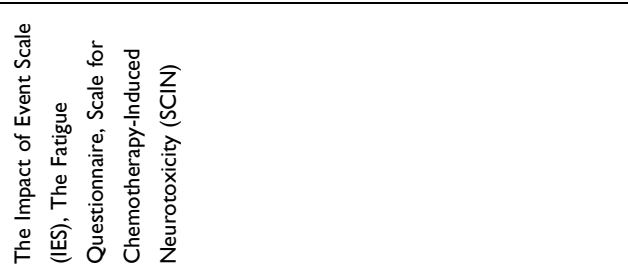 \\
\hline 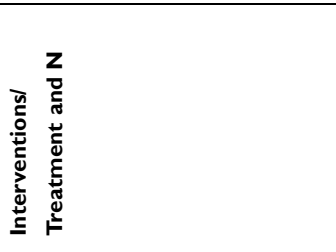 & 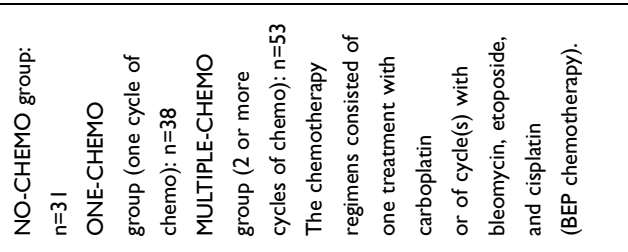 \\
\hline & 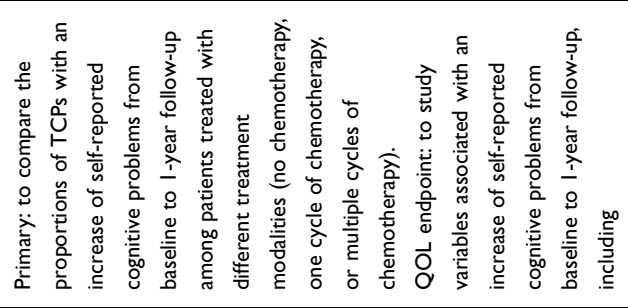 \\
\hline 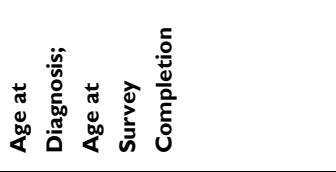 & 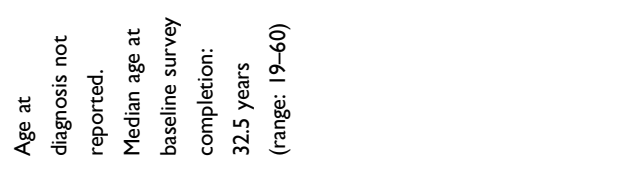 \\
\hline 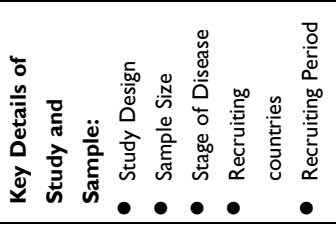 & 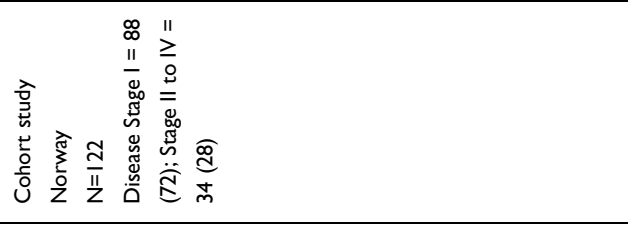 \\
\hline 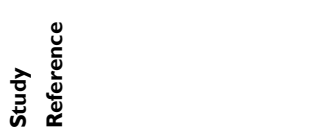 & 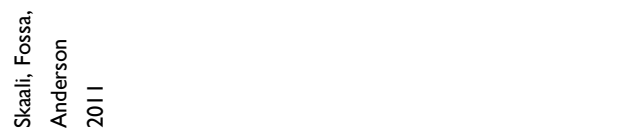 \\
\hline
\end{tabular}




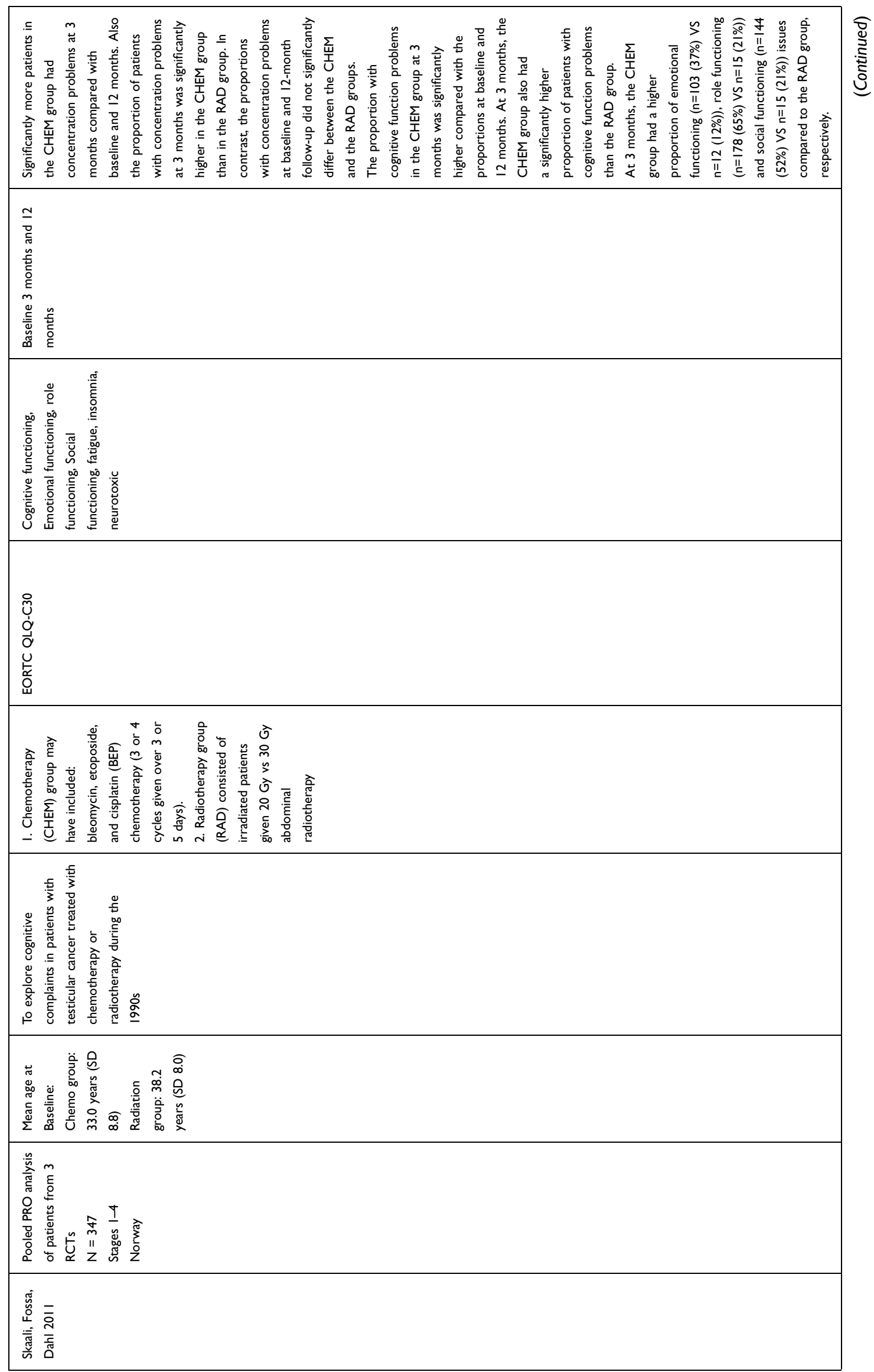




\begin{tabular}{|c|c|}
\hline 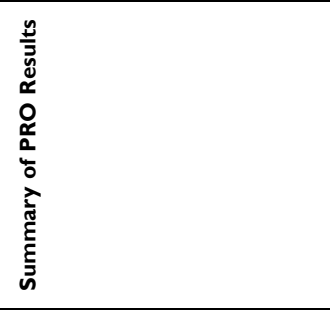 & 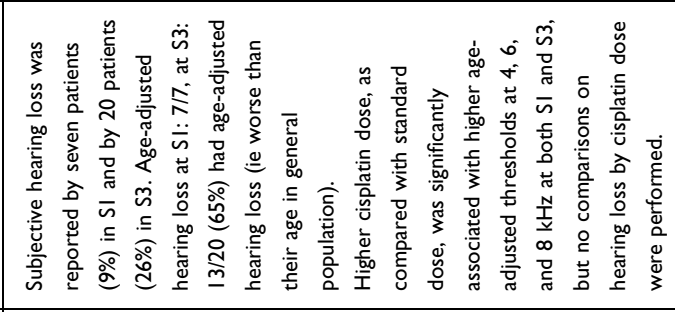 \\
\hline 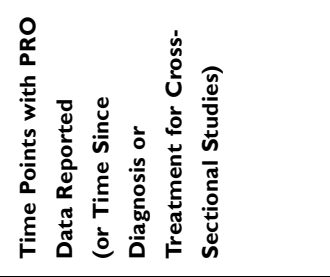 & 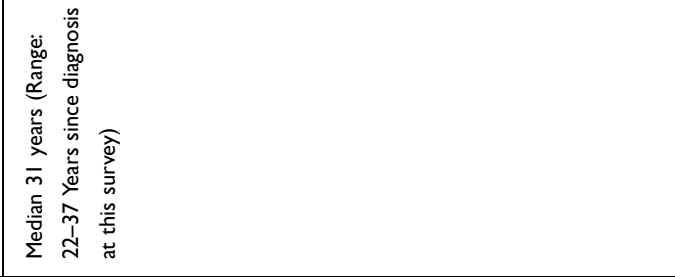 \\
\hline 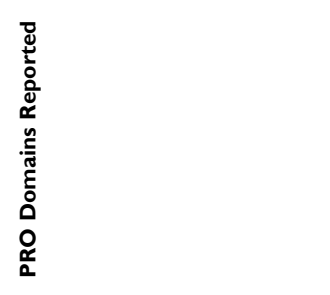 & 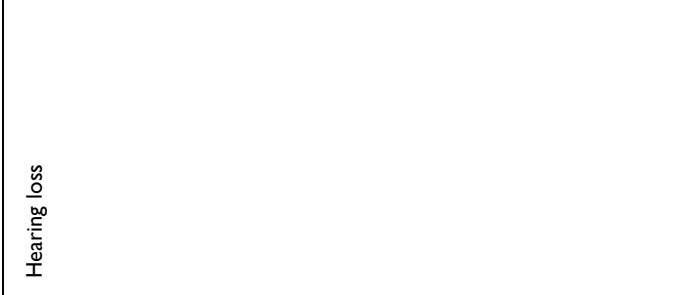 \\
\hline 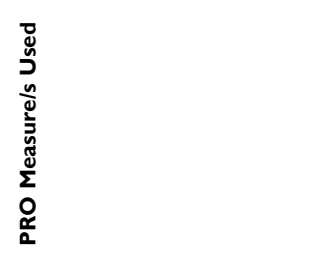 & 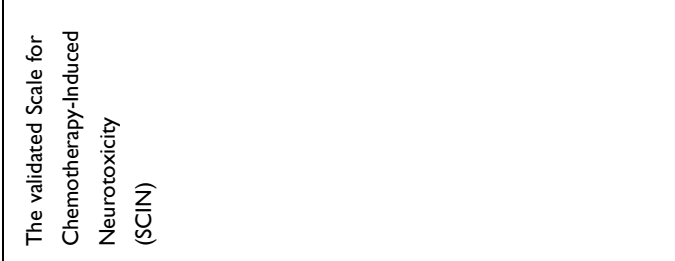 \\
\hline 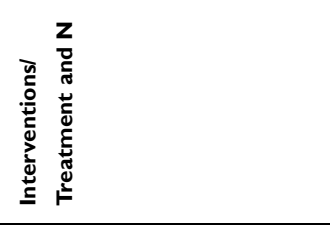 & 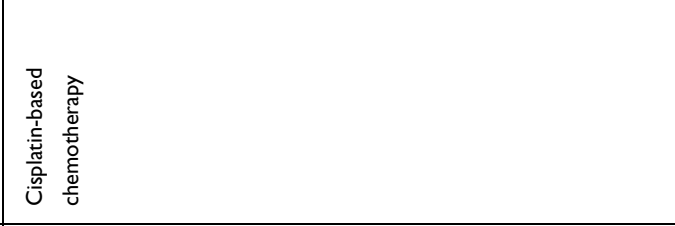 \\
\hline 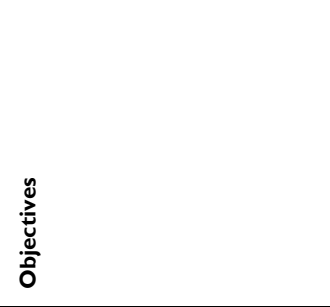 & 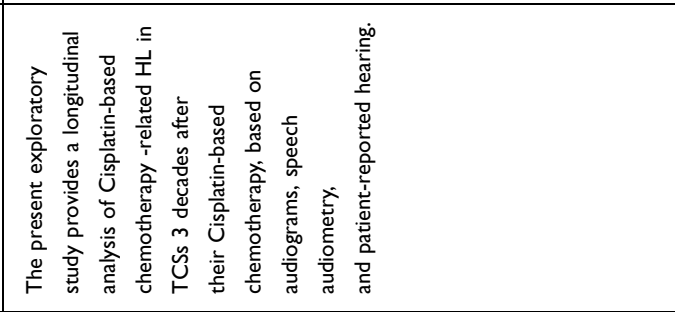 \\
\hline 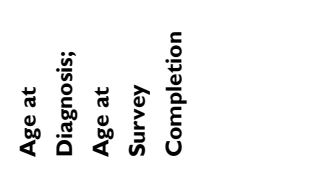 & 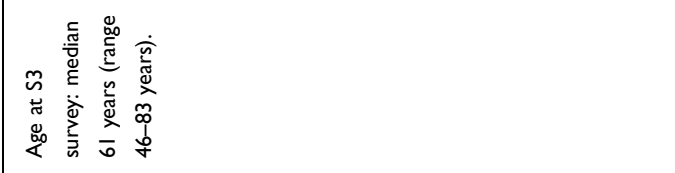 \\
\hline 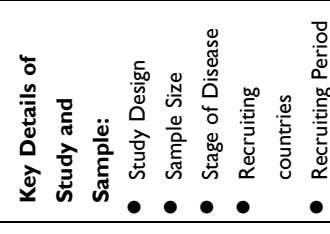 & 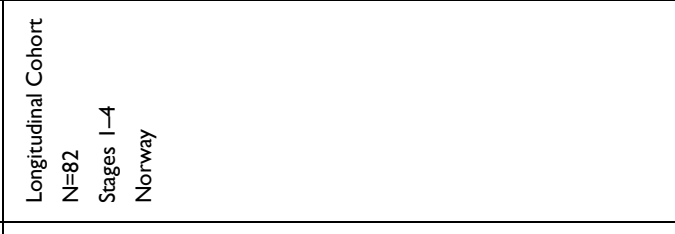 \\
\hline 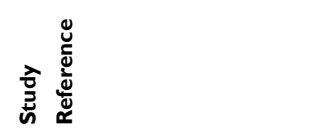 & 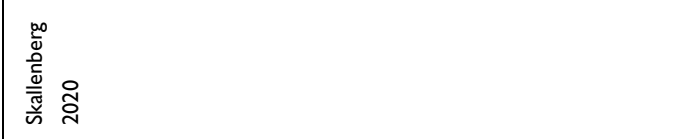 \\
\hline
\end{tabular}




\begin{tabular}{|c|c|}
\hline 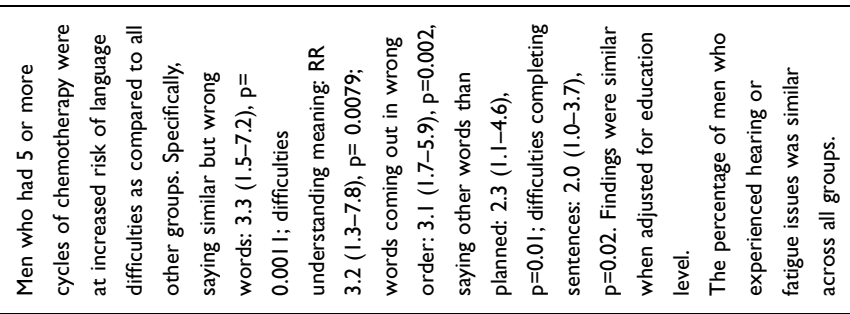 & 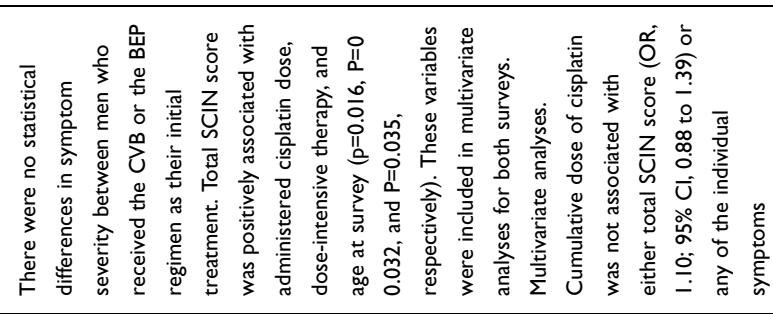 \\
\hline 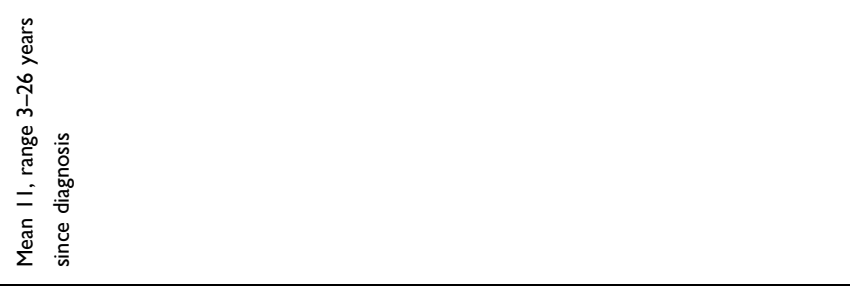 & 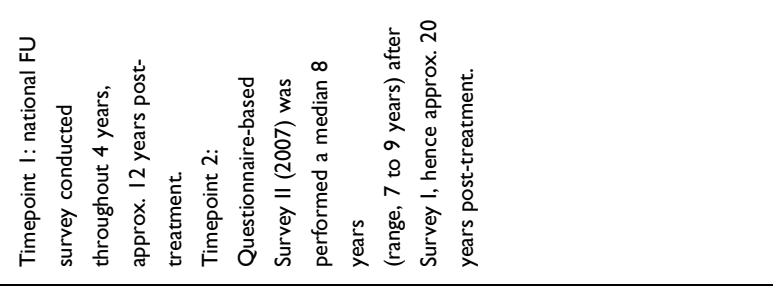 \\
\hline 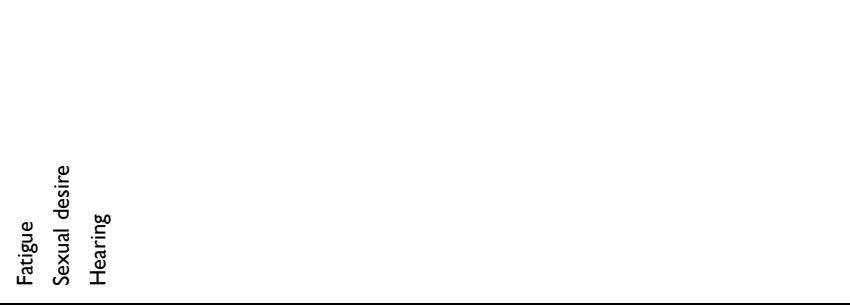 & 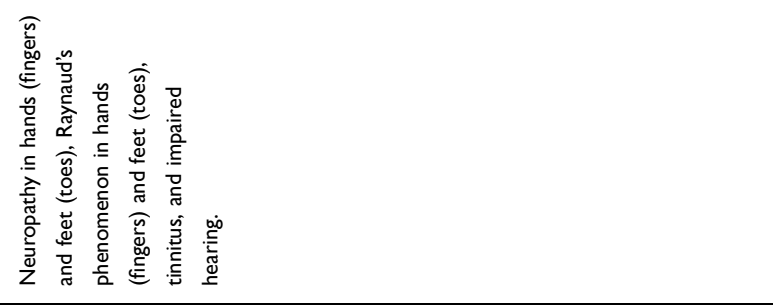 \\
\hline 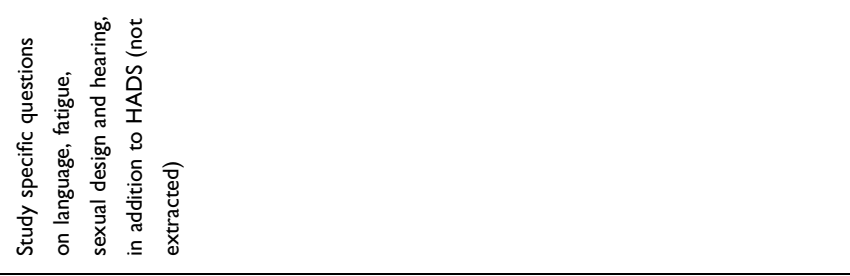 & 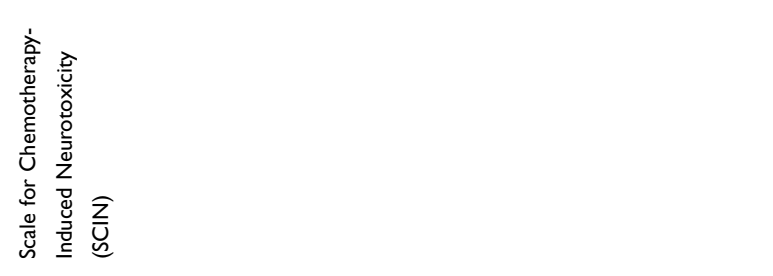 \\
\hline 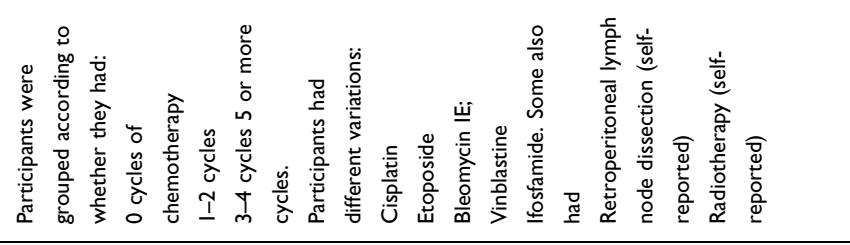 & 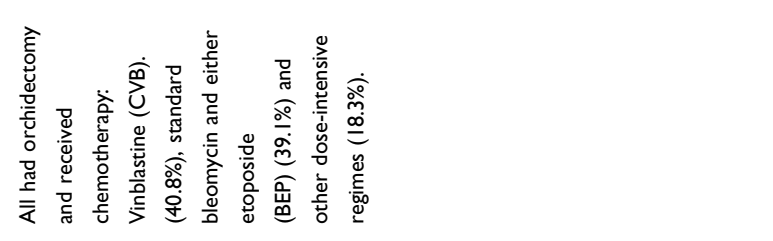 \\
\hline 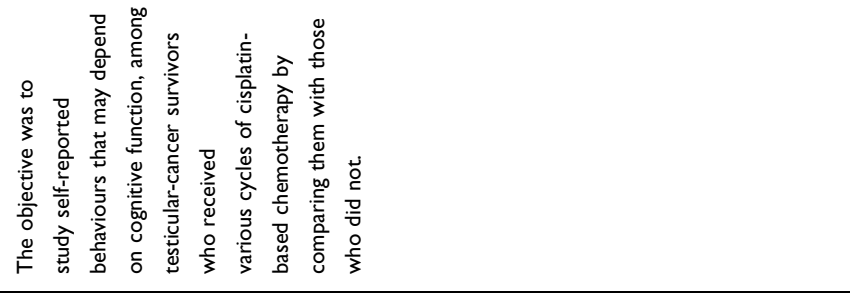 & 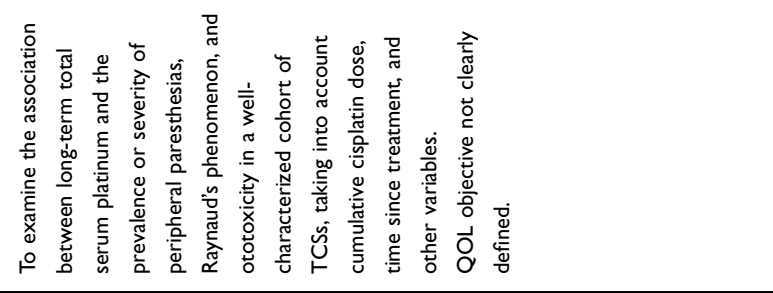 \\
\hline 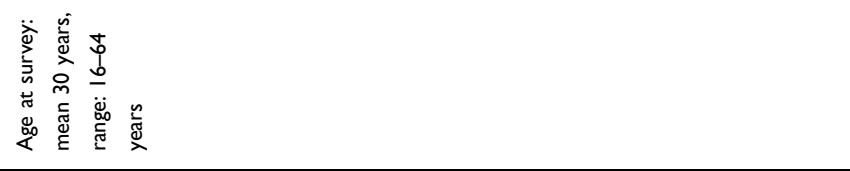 & 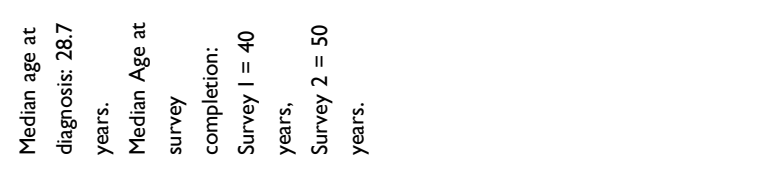 \\
\hline 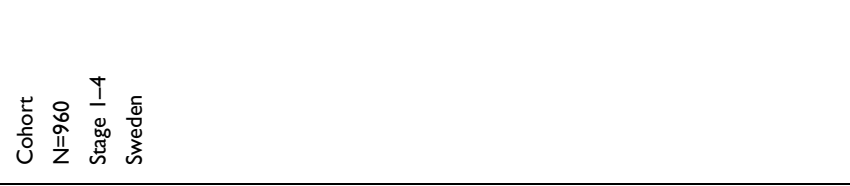 & 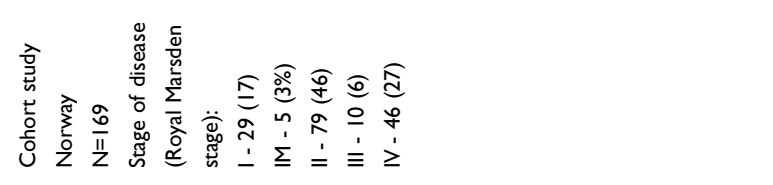 \\
\hline & 商 \\
\hline
\end{tabular}




\begin{tabular}{|c|c|}
\hline 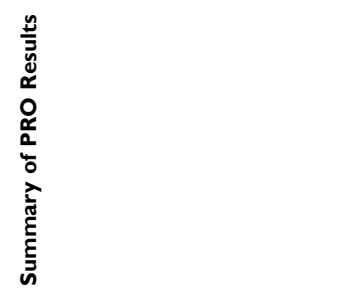 & 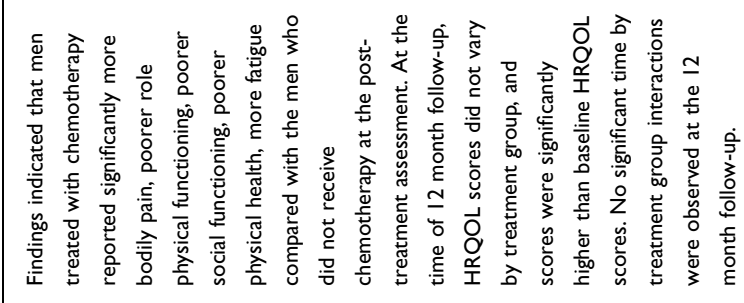 \\
\hline 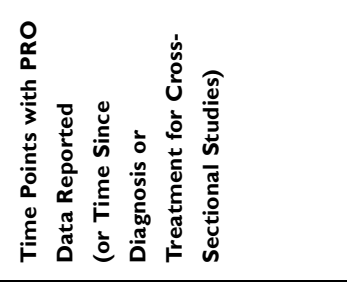 & 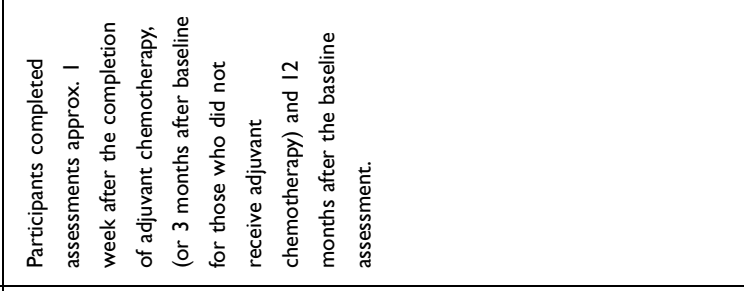 \\
\hline 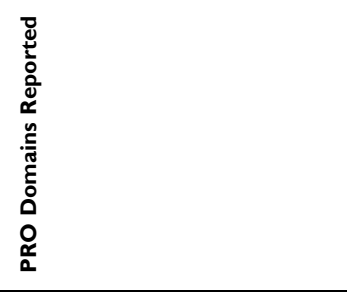 & 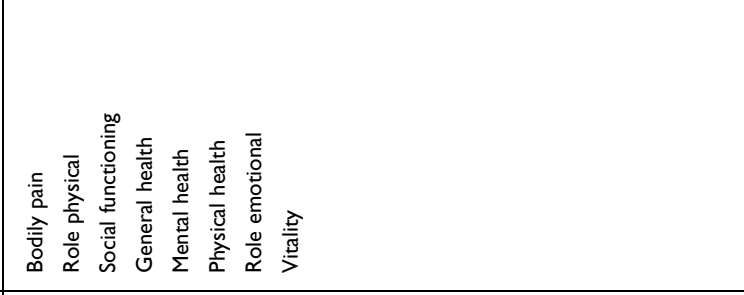 \\
\hline 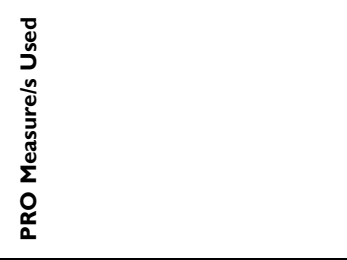 & 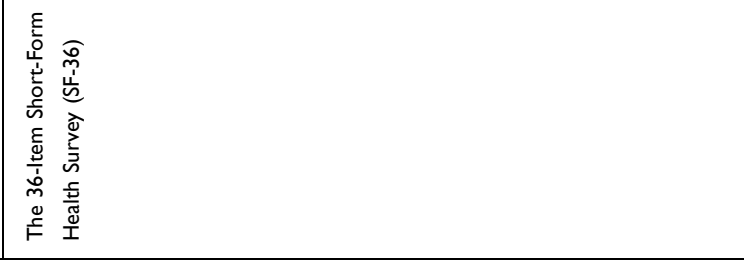 \\
\hline 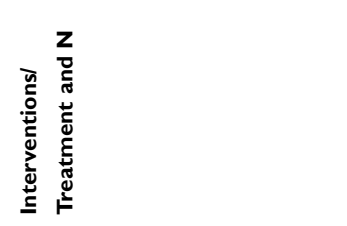 & 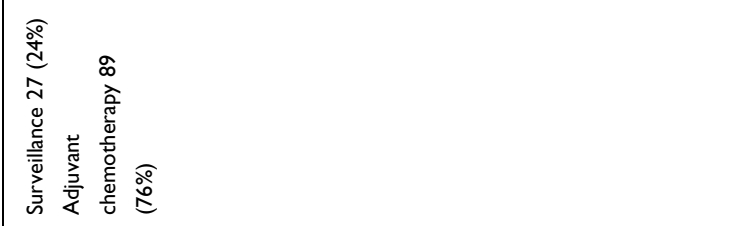 \\
\hline & 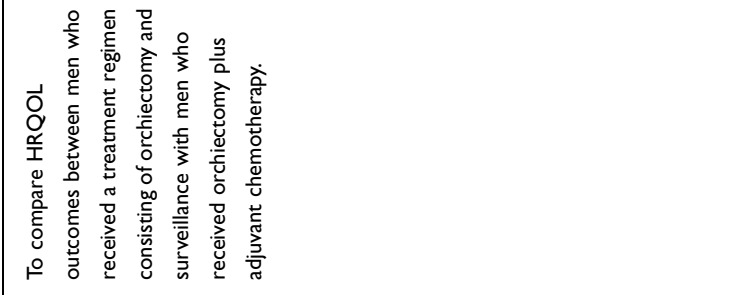 \\
\hline 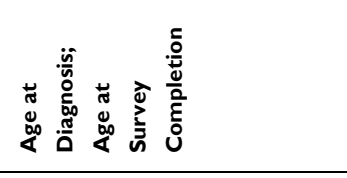 & 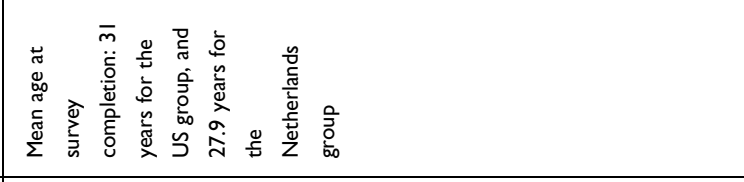 \\
\hline 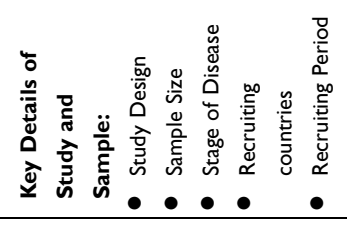 & 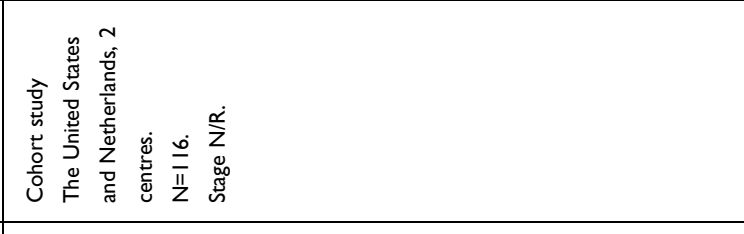 \\
\hline 旁彥产 & 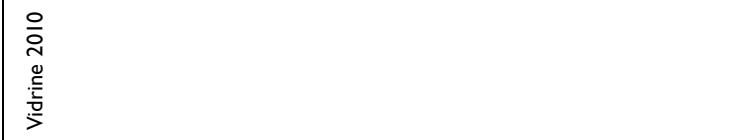 \\
\hline
\end{tabular}



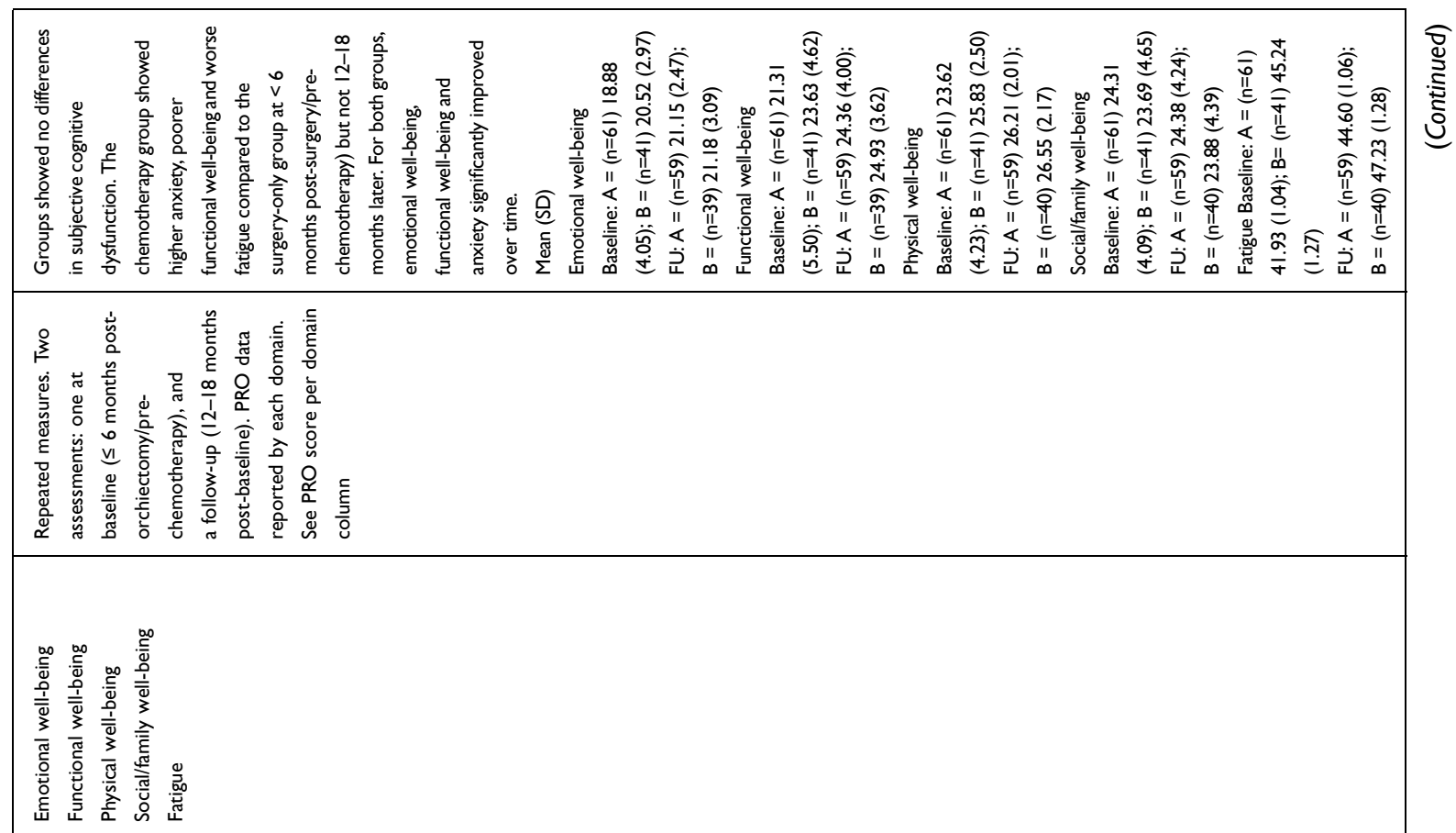

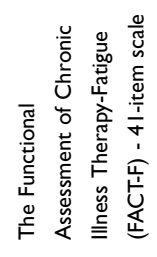
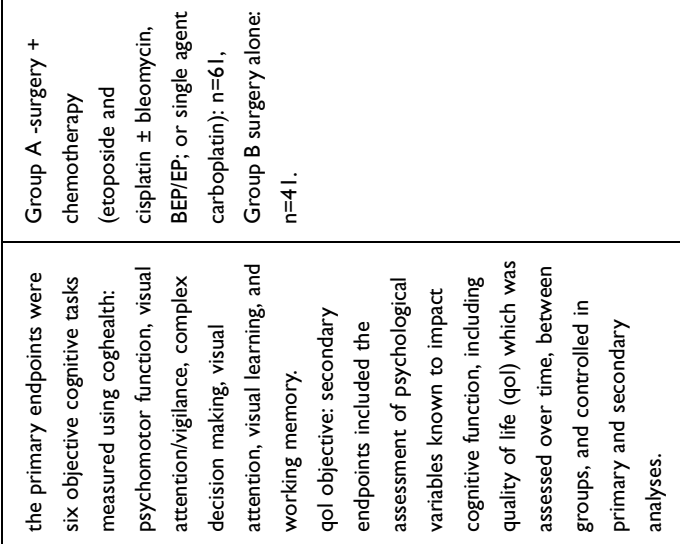

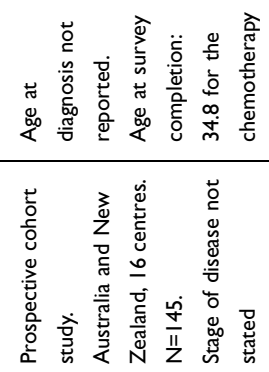

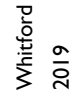




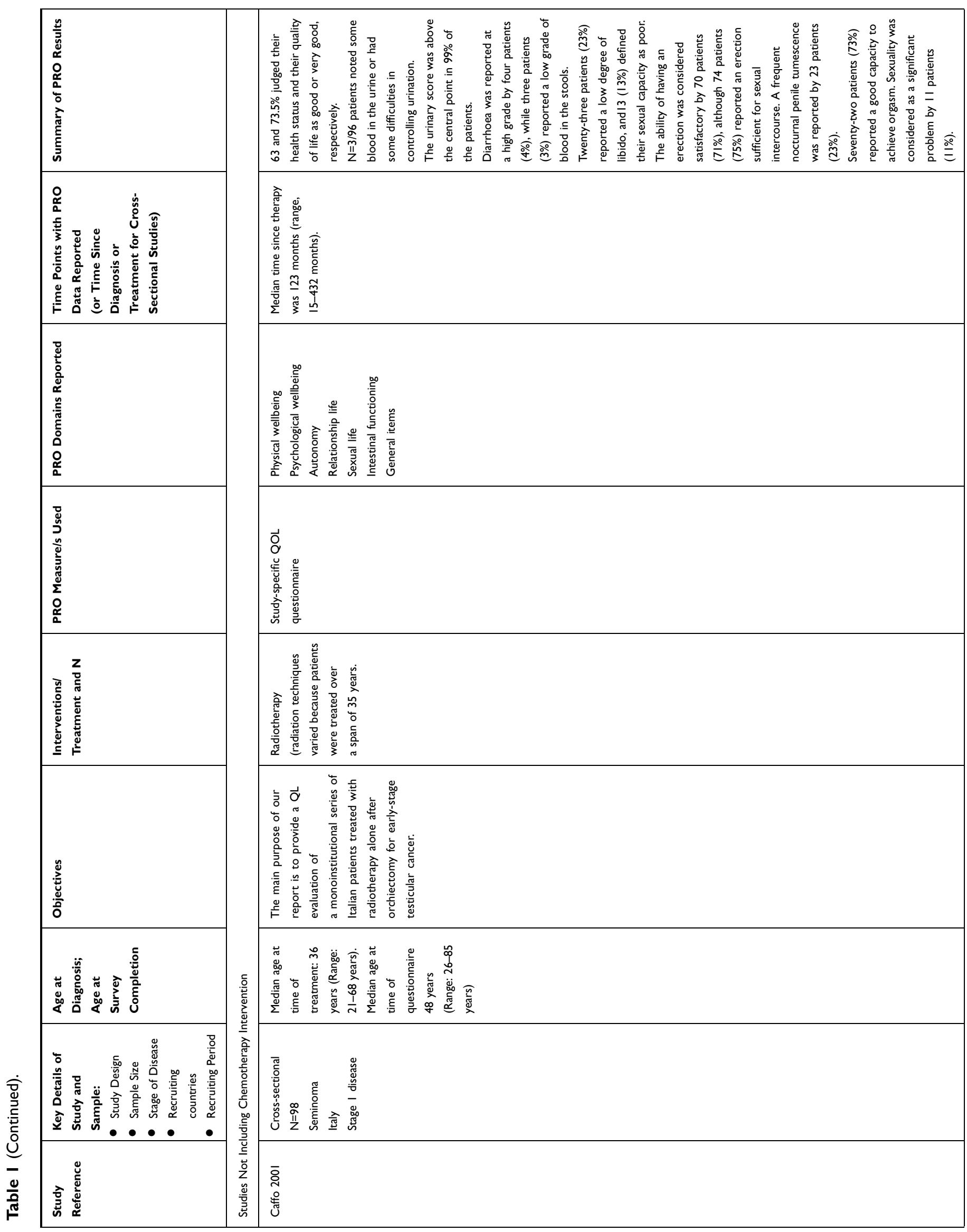




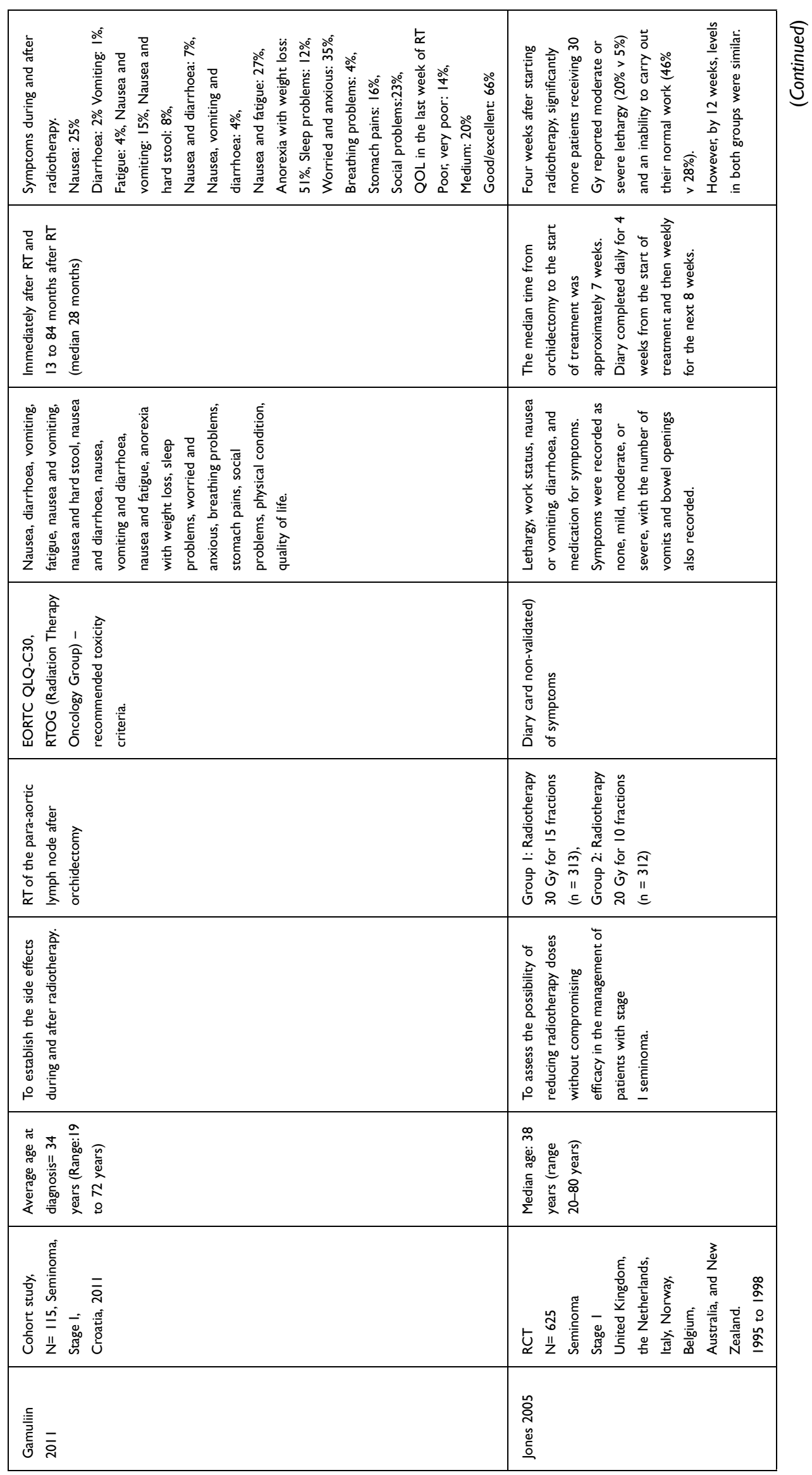




\begin{tabular}{|c|c|}
\hline 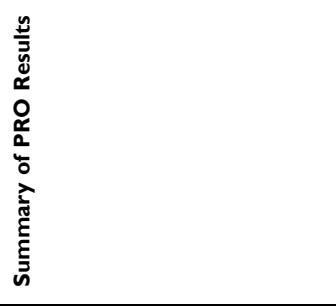 & 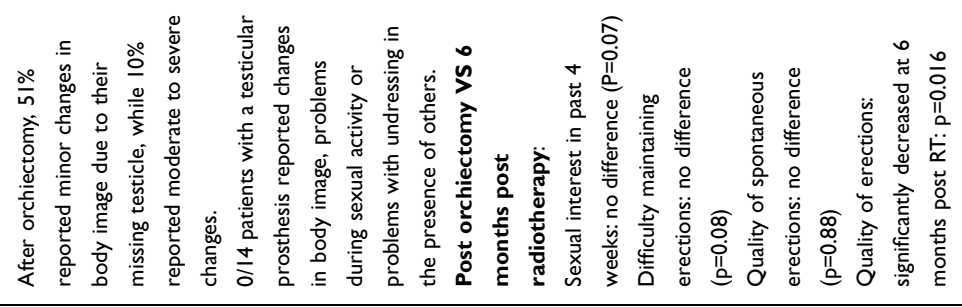 \\
\hline 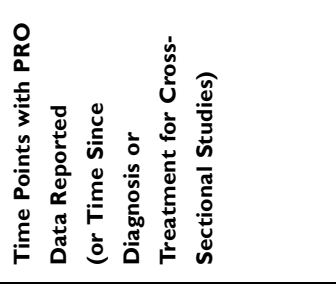 & 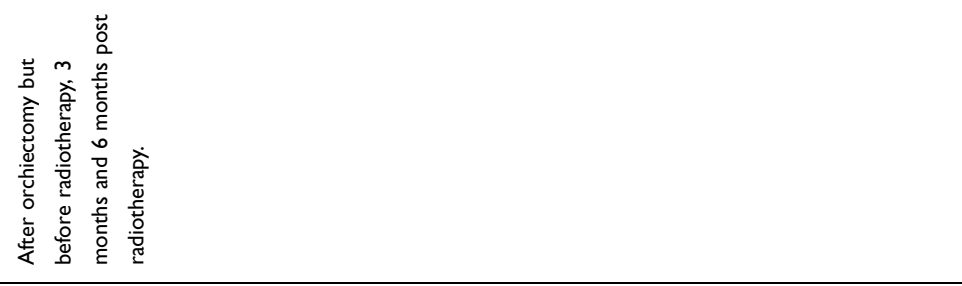 \\
\hline 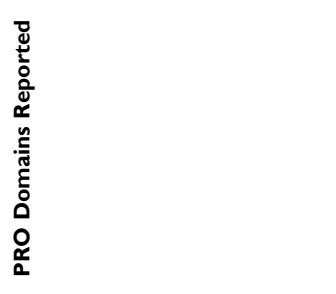 & 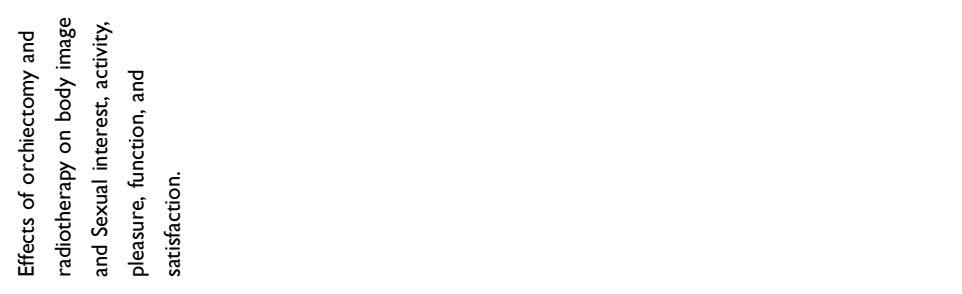 \\
\hline 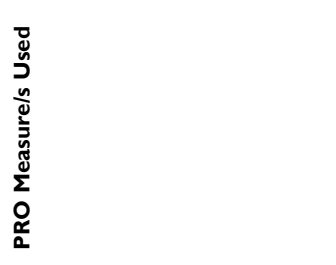 & 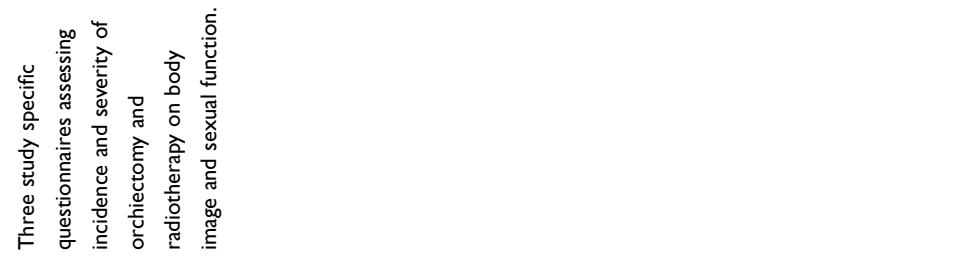 \\
\hline 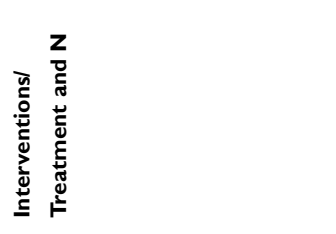 & 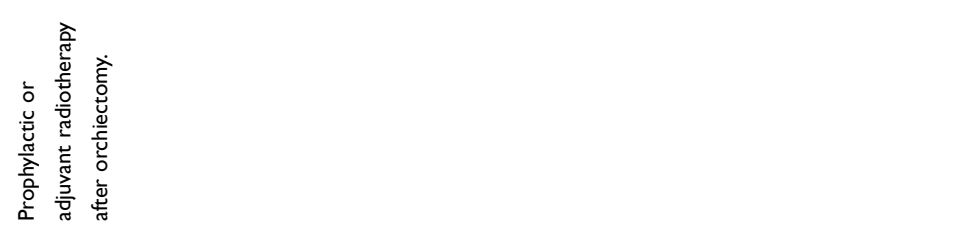 \\
\hline & 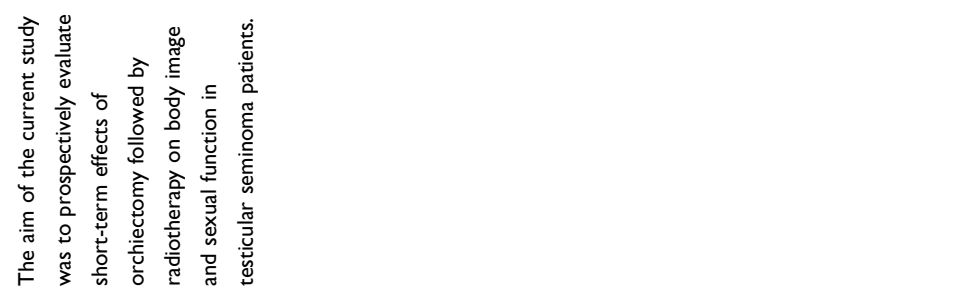 \\
\hline 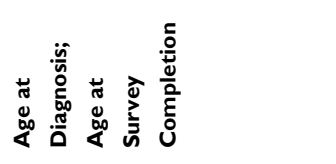 & 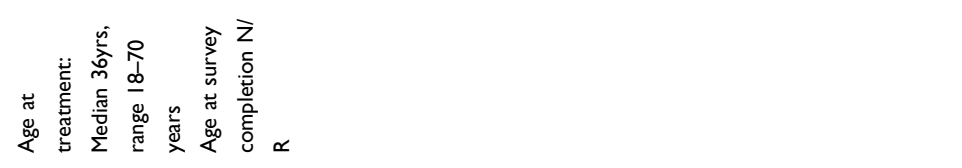 \\
\hline 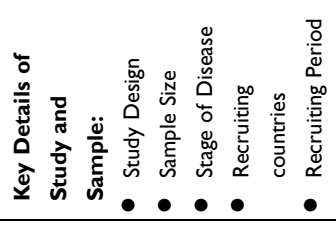 & 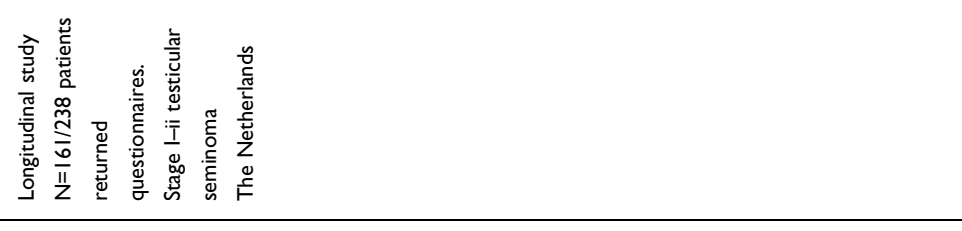 \\
\hline 츨 & 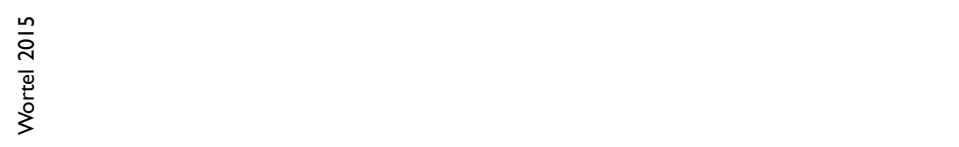 \\
\hline
\end{tabular}




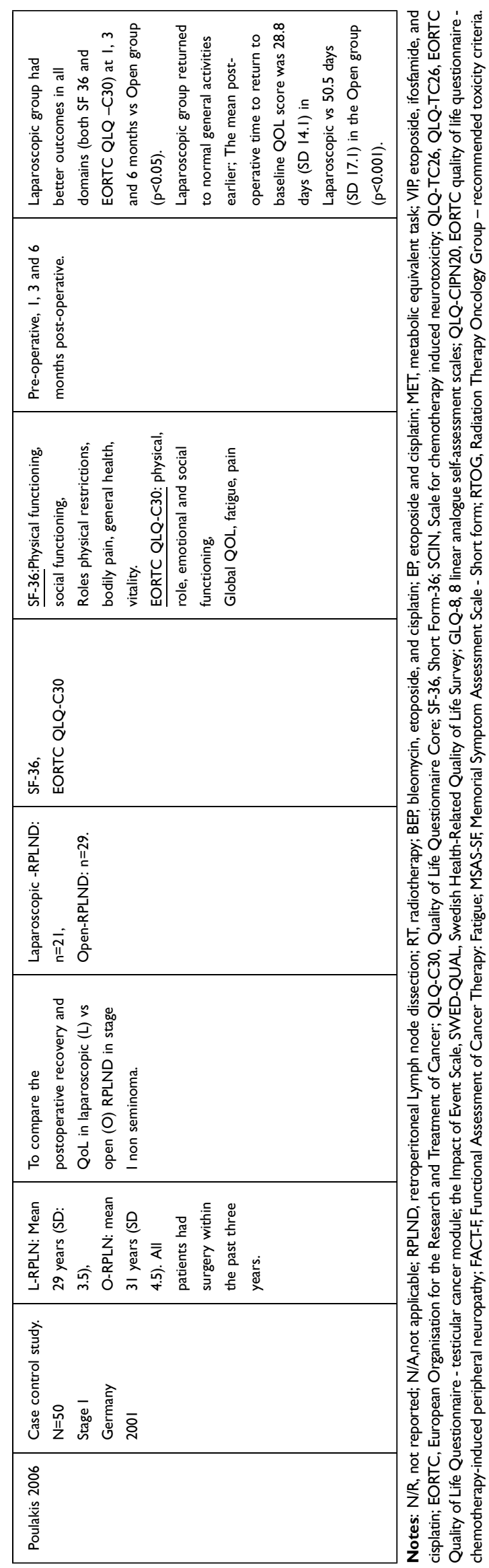


$(\mathrm{p}=0.003) .{ }^{29}$ Oldenburg $2006(\mathrm{~N}=684)$ found that lower proportions of men who had low-dose chemotherapy reported "high" SCIN scores (28-37\%, compared to $63-72 \%$ in the high-dose chemotherapy group). "Low" SCIN scores were found in $53-54 \%$ of men in the lowdose group compared to $46-47 \%$ in the high-dose group. ${ }^{19}$

Regarding dose-induced ototoxicity, one study $(\mathrm{N}=812)$ found that among men treated with four cycles of bleomycin, etoposide, and cisplatin (BEP), there were significantly higher rates of tinnitus for those treated over three days as compared with five days $(p=0.0002) .{ }^{24}$ Oldenburg $2007(\mathrm{~N}=238)$ also found that cumulative dose of cisplatin was significantly associated with tinnitus $(p<0.001)$ and hearing impairment $(p<0.001)$, along with the other neurotoxic side-effects noted above.

\section{Differences in Neurotoxicity and Raynaud-Like Phenomena by Treatment Type}

BEP seemed to be associated with some higher neurotoxicity than other regimens. The prevalence and severity of Raynaud's phenomenon after three cycles of BEP exceeded those for four cycles of etoposide, and cisplatin (EP) ${ }^{34}$ Fung $(\mathrm{N}=952)$ also found higher toxicity associated with BEP; specifically that the type and prevalence of individual adverse health outcomes after EP x 4 cycles versus BEP X 3 cycles were similar ( $p=0.05)$, except for Raynaud phenomenon ( $11.6 \%$ v $21.4 \% ; \mathrm{P}=0.01)$, peripheral neuropathy $(29.2 \% \vee 21.4 \% ; \mathrm{p}=0.02)$, and obesity $(25.5 \%$ v $33.0 \% ; \mathrm{p}=0.04){ }^{48}$

Chemotherapy appeared to be associated with more neurotoxic symptoms compared to other types of treatment, including surveillance, ${ }^{23,39}$ RPLND, or radiotherapy. ${ }^{23,37}$ Neurotoxic symptoms were, however, associated with other treatments as well. Fossa 1996 found that although participants treated with radiotherapy or chemotherapy had the highest scores for neurological symptoms, Raynaud-like phenomena and ototoxicity, participants treated with surveillance also experienced these symptoms at lower levels. ${ }^{27}$ Oldenburg $2006(\mathrm{~N}=684)$ also found a higher proportion of irradiated patients with "pain, paresthesias in the feet/toes" compared to men who had surgery alone. ${ }^{19}$

Ototoxicity was also reported for different modalities. Oldenburg et al also found $10 \%$ of the radiotherapy group reported "very much" tinnitus or impaired hearing. ${ }^{19}$ Compared to age-matched controls, the rate of hearing loss was worse for testicular cancer survivors treated with cisplatin. ${ }^{42}$

\section{Functional and Symptom Scales}

Seven studies reported differences in functional and symptom scales according to treatment modality ${ }^{35,40,45,46}$ or higher doses of treatment, ${ }^{24,26,32}$ at one or more assessment time points. Kim et al found that survivors diagnosed five or more years ago treated with chemotherapy had poorest scores (compared to radiotherapy and surgery) on the SF-36 physical $(\mathrm{p}=0.0032)$ and mental component scores $(p=0.039)$, physical functioning $(p=0.027)$, rolephysical $(p=0.0044)$, general health $(p=0.0001)$, vitality $(\mathrm{p}=0.011)$, social functioning $(\mathrm{p}=0.016)$ and mental health scales $(p=0.028){ }^{35}$

Two studies found that men treated with chemotherapy reported worse functioning (physical functioning, social functioning ${ }^{45}$ and functional wellbeing ${ }^{46}$ ) and worse fatigue $^{45,46}$ compared to men who did not receive chemotherapy initially, but there were no between-group differences $12-18$ months later in either study.

\section{Functional and Symptom Scales by Chemotherapy Dose or Concurrent Treatment}

Chemotherapy dose or concurrent treatment was related to higher levels of self-reported toxicity in three of the highquality studies. An RCT by de Witt and colleagues $(\mathrm{N}=812)$ found significant differences in favour of three cycles of BEP (versus four cycles of BEP) for physical function ( $p=0.008)$, role function $(p=0.03)$, cognitive function $(p=0.04)$, fatigue $(p=0.003)$, nausea/vomiting $(\mathrm{p}=0.008)$, appetite loss $(\mathrm{p}=0.001)$, and overall QOL $(p=0.03)$ at three months which decreased to no difference at 12 months. $^{24}$ In a RCT by Fossa and colleagues $(\mathrm{N}=666)$, men randomised to four cycles of chemotherapy for three days had more nausea/vomiting as assessed by the QLQ-C30 compared to men who had three cycles for three days, at three months. The lowest mean nausea/ vomiting scores were seen for the group who had three cycles for five days. ${ }^{26}$ In another study of survivors diagnosed an average of 10 years ago, men who had chemotherapy + radical resection had higher levels of nausea/vomiting than men who had chemotherapy only $(p=0.04)$, but results were not clinically significant. ${ }^{40}$

\section{Functional and Symptom Scales Associated with Radiotherapy}

Three high-quality studies reported the impact of radiotherapy on functional and symptom scales. In one study $(\mathrm{N}=625)$, significantly more patients receiving $30 \mathrm{~Gy}$ for 15 fractions (compared to men receiving 20 Gy for 10 
fractions) reported moderate or severe lethargy $(20 \%$ $\mathrm{v} 5 \%$ ) and an inability to carry out their normal work (46\% v 28\%) four weeks after starting radiotherapy. However, by 12 weeks, levels in both groups were similar. $^{32}$ In a descriptive radiotherapy study $(\mathrm{N}=98)$, bowel side-effects were described. Diarrhoea was reported at a high grade by $4 / 99$ patients $(4 \%)$ while three patients (3\%) reported a low grade of blood in the stools. ${ }^{11}$

Wortel $2015(\mathrm{~N}=161)$ reported no impact on body image or problems with undressing in the presence of others in the first six months since radiotherapy treatment.

\section{Studies Reporting No Differences by Treatment}

Three high-quality studies found no effect on self-reported functioning and symptoms based on treatment. Oechsle $(\mathrm{N}=164)$ found no differences on any Memorial Symptom Assessment Scale-Short Form scale among survivors treated with surgery versus radiotherapy versus chemotherapy in a long-term follow-up analysis. ${ }^{38}$ Miyake ( $\mathrm{N}=102)$ found no significant differences in any SF-36 scale score between patients treated with chemotherapy versus surveillance who had not shown any evidence of disease for at least six months. ${ }^{36}$ Flechtner found no significant differences on any QLQ-C30 scale at three-years post-treatment between men treated with BEP versus RPLND. ${ }^{25}$

\section{Cognitive Functioning}

Chemotherapy was significantly associated with cognitive problems in two of the 29 high-quality studies. Skaali and colleagues $(\mathrm{N}=122)$ found more severe cognitive problems in the first 12 months since diagnosis among men who had one cycle $(29 \%)$ and multiple cycles $(25 \%)$ of chemotherapy, compared to those who had no chemotherapy (3\%). ${ }^{41}$ Similarly, survivors who were on average 11 years postdiagnosis who had five or more cycles of chemotherapy were at increased risk of self-reported language difficulties as compared to men who had fewer cycles or no chemotherapy. $^{43}$

\section{Sexual Functioning}

Five high-quality studies described the impact of treatment on sexual functioning. Only one of these studies found no relationship between type of treatment and modification of sexual life among survivors who were an average of 11 years since diagnosis. ${ }^{31}$ The other four studies observed differences by treatment. Jonker-Poole $(\mathrm{N}=264)$ found that surveillance had the lowest impact on sexual functioning and activity among survivors who were a mean of 6.7 years post-diagnosis. Chemotherapy was associated with problems with libido $(p=0.03)$, orgasm $(p=0.010)$, ejaculation $(p=0.04)$, and sexual activity $(p=0.005)$ after age was adjusted for, among these survivors. Ejaculation problems were worse among men who had chemotherapy + RPLND compared to men who had radiotherapy $(\mathrm{p}<0.001)$ or chemotherapy $(p=0.002) .^{33}$

Similarly, Huddart $(\mathrm{N}=680)$ found that men who had chemo-radiotherapy more than five years previously were more likely to report less interest in $\operatorname{sex}(p=0.01)$ compared to men who had chemotherapy alone, radiotherapy or surveillance. ${ }^{30}$ Men who had radiotherapy reported less sexual activity $(\mathrm{p}=0.051)$ and reduced sexual enjoyment $(p=0.05)$ compared to surveillance. Survivors who had chemotherapy, as compared to men who had surveillance, reported more worries about fathering children $(\mathrm{p}=0.009)^{30}$

In a single-arm radiotherapy study, Wortel $(\mathrm{N}=161)$ found that the self-reported quality of men's erections decreased from post-orchidectomy to 6 months postradiotherapy $(p=0.016)$. No other impacts of radiotherapy were seen at six months post-radiotherapy for sexual interest $(p=0.07)$, maintaining erections $(p=0.08)$, or quality of spontaneous erections $(\mathrm{p}=0.88){ }^{47}$ In another study of radiotherapy impacts $(\mathrm{N}=98), \mathrm{n}=23(23 \%)$ of participants reported a low degree of libido, $13(13 \%)$ defined their sexual capacity as poor, 74 patients (75\%) reported an erection sufficient for sexual intercourse, and 72 patients (73\%) reported a good capacity to achieve orgasm. Sexuality was considered a significant problem by 11 patients $(11 \%){ }^{11}$

\section{Discussion}

This review summarises the patient-reported symptoms, side-effects and functional impacts of common treatments for testicular cancer. In balance, it appears that chemotherapy is associated with higher levels of side-effect burden than other treatments, and the burden is increased with increasing dose of chemotherapy. Raynaud's phenomena and neurotoxic side-effects, such as hearing issues and peripheral neuropathy seemed to be associated more frequently and more intensely with chemotherapy than with other forms of treatment, although we did not conduct a meta-analysis to verify this statistically. Although only two high-quality studies that met our inclusion criteria studied the impact of treatment on cognitive functioning, both studies showed evidence of higher doses of 
chemotherapy being associated with more cognitive problems. ${ }^{41,43}$

The evidence for differing impact of treatment on sexual functioning was mixed. Chemotherapy, alone or in combination with other modalities, was associated with lower sexual interest in two studies, ${ }^{30,33}$ yet another study reported no difference in this regard. ${ }^{31}$ Radiotherapy, alone or in combination with other modalities, was frequently associated with less interest in sex, reduced sexual activity and poorer-quality erections, ${ }^{11,30,47}$ although no impact of radiotherapy on body image or undressing in front of others was found. ${ }^{47}$ None of the included, high-quality studies explicitly examined the impact of a bilateral orchiectomy on sexual functioning.

Differences in functional and symptom scales according to treatment modality ${ }^{35,40,45,46}$ or higher doses of treatment ${ }^{24,26,32}$ were frequently reported. Nausea and vomiting were frequently reported by men who received chemotherapy, during active treatment and shortly after ${ }^{24,26}$ or radical (retroperitoneal lymph node dissection) following cisplatin-based chemotherapy among survivors a median of 10 years since treatment-. ${ }^{40}$ Chemotherapy was associated with worse physical functioning, social functioning, ${ }^{45}$ functional wellbeing ${ }^{46}$ and fatigue ${ }^{45,46}$ compared to men who did not receive chemotherapy in the first six-months post-treatment.

Differences seen in results between studies may be an effect of different treatment regimens, but it is important to note that they may be an effect of methodological differences. There were 27 different PRO measures used across the 41 studies, and only 14 of these appeared to be validated, which may reflect the quality of these measures. Some of these measures focused exclusively on neurotoxic effects (SCIN, QLQ-CIPN20) whereas others focused on more general functional issues and side-effects (eg, SF-36, QLQ-C30, QLQ-TC26). Had the included studies measured the same set of PRO domains, our findings may have been different. The data collection time points also differed, depending on the specific research questions of the individual studies. We noted a trend for differences seen during active treatment, or shortly thereafter, to reduce or disappear over time.

We also note that most of the studies that met our inclusion criteria (36 of 41 studies in total, and 26 of the high-quality studies) included chemotherapy; therefore, our results may be skewed to reflect the burden associated with chemotherapy over other treatment modalities.
It is important to note that certain treatments featured in this review are no longer recommended in clinical practice guidelines, due to more effective treatments becoming available. For example, one study (which did not meet our quality criteria for inclusion in the narrative synthesis) reported use of vincristine, ${ }^{49}$ which is no longer used. According to most guidelines, ${ }^{3}$ BEP or EP chemotherapy is standard for all stages of testicular cancer. Moreover, improved surgical techniques have been found to preserve sexual function and reduce short- and longterm toxicities. ${ }^{50,51}$ For instance, bilateral RPLND was associated with retrograde ejaculation; however, modified unilateral RPLND reduced the frequency of this problem. Radiotherapy doses, fields, indications, and use has diminished. Studies conducted before 2000, two of which are included in our narrative synthesis, ${ }^{27,33}$ used higher doses and larger fields of radiotherapy, which may have contributed to more gastrointestinal or other toxicities.

The strengths of this study include a robust search strategy that was developed in collaboration with an academic librarian and pilot tested several times, and use of established and trusted review methods. ${ }^{15,16}$ We limited the search to English articles, which means we may have missed some relevant, non-English literature; however, we do not anticipate that this would impact our findings, as previous research suggests. ${ }^{52}$ We also note that many $(n=27 / 41)$ of the included studies recruited participants from Western Europe. This is consistent with the incidence of testicular cancer being higher in Western Europe, as well as the UK, Australia and New Zealand. ${ }^{2}$ We acknowledge the possibility that we may have missed relevant articles due to human error. We also note that there may have been overlap in the samples of Norwegian studies,,${ }^{9,44}$ as these participants were recruited from the same program of research

It can be difficult to collate and compare the PRO results of different studies due to variation in overall quality, designs, measures, analysis techniques and reporting formats. This is a recognised issue ${ }^{53}$ and numerous PRO methodological researchers are taking steps to make PRO data more accessible and interpretable to readers, through the development of guidelines for design, ${ }^{54}$ conduct, $^{55}$ analysis $^{56}$ and reporting ${ }^{57}$ of PROs. We encourage researchers to read and adhere to these PRO guidelines when planning and conducting research on men with a history of testicular cancer with PROs. It is not always appropriate to recommend the same PRO measures for all studies, as the PROs of 
importance to a particular clinical context are often nuanced and context-specific. Having a set of standard PROs appropriate to all cancers is helpful, with the addition of disease- or treatment-specific PROs as relevant, would be a great approach to improve transparency and interpretability of patient-centred outcomes. More importantly, involving patients in the development of measures ensures that key issues are addressed relating to the specific disease and treatment options, and that questions are acceptable to patients. We did not assess the development methodology of the measures included in this review; however, we do note that the EORTC PRO measures involve patients in their development.

Differences in follow-up time points for PRO assessment can also present challenges for interpretation. The survivorship studies in this review had very wide data collection windows. Although treatment side-effects may ease over time, reporting mean scores from participants at very different survivorship stages may be misleading. We chose to include data from studies of active treatment and survivorship to canvas the experience of testicular cancer treatment and recovery more comprehensively, and have noted the time since treatment where available, for transparency.

This review summarises the expected side-effects, both short-term and long-term, of treatments used commonly for testicular cancer. While many studies found that shortterm adverse effects resolved within 12 months of treatment, there were clearly long-term effects, often dosedependent, associated with chemotherapy and radiotherapy. It is widely acknowledged that patients have varying treatment preferences; while some prioritise length of life at all costs, others consider risk and fear of recurrence as well as quality of life to varying degrees. Offering information about all of these aspects upon considering treatment options allows patients, their families and clinicians to be informed, prepared and empowered to make treatment decisions suited to their preferences and personal situations. We hope that the findings of this review will assist this process.

\section{Acknowledgments}

Professor Fabio Efficace and Francesco Sparano from GIMEMA, Rome, Italy, for their kind assistance searching the PROMOTION database. Our academic librarian, Roderick Dyson from the University of Sydney,
Australia, for his assistance in developing the search strategy.

\section{Disclosure}

Dr Rebecca Mercieca-Bebber reports grants from Patient Voice Matters, outside the submitted work. The authors report no other conflicts of interest in this work.

\section{References}

1. Chia VM, Quraishi SM, Devesa SS, Purdue MP, Cook MB, McGlynn KA. International trends in the incidence of testicular cancer, 1973-2002. Cancer Epidemiol Biomarkers Prev. 2010;19 (5):1151-1159. doi:10.1158/1055-9965.EPI-10-0031

2. Park JS, Kim J, Elghiaty A, Ham WS. Recent global trends in testicular cancer incidence and mortality. Medicine. 2018;97(37): e12390. doi:10.1097/MD.0000000000012390

3. Oldenburg J, Fosså SD, Nuver J, et al. Testicular seminoma and non-seminoma: ESMO clinical practice guidelines for diagnosis, treatment and follow-up. Ann Oncol. 2013;24(Suppl 6):vi125-32. doi:10.1093/annonc/mdt304

4. NIH National Cancer Institute. Cancer stat facts: testicular cancer; 2021. Available from: https://seer.cancer.gov/statfacts/html/testis. html. Accessed May 13, 2021.

5. Jacobsen KD, Theodorsen L, Fossa SD. Spermatogenesis after unilateral orchiectomy for testicular cancer in patients following surveillance policy. J Urol. 2001;165(1):93-96. doi:10.1097/00005392200101000-00023

6. Shpunt I, Leibovici D, Ikher S, et al. Spermatogenesis in testicles with germ cell tumors. Isr Med Assoc J. 2018;20(10):642-644.

7. Donohue JP, Foster RS, Rowland RG, Bihrle R, Jones J, Geier G. Nerve-sparing retroperitoneal lymphadenectomy with preservation of ejaculation. J Urol. 1990;144(2 Pt 1):287-91; discussion 91-2. doi:10.1016/S0022-5347(17)39434-X

8. Rossen P, Pedersen AF, Zachariae R, von der Maase H. Sexuality and body image in long-term survivors of testicular cancer. Eur J Cancer. 2012;48(4):571-578. doi:10.1016/j.ejca.2011.11.029

9. Oldenburg J, Kraggerud SM, Brydoy M, Cvancarova M, Lothe RA, Fossa SD. Association between long-term neuro-toxicities in testicular cancer survivors and polymorphisms in glutathione-s-transferaseP1 and -M1, a retrospective cross sectional study. J Transl Med. 2007;5:1-8. doi:10.1186/1479-5876-5-70

10. Huddart RA, Norman A, Shahidi M, et al. Cardiovascular disease as a long-term complication of treatment for testicular cancer. J Clin Oncol. 2003;21(8):1513-1523. doi:10.1200/JCO. 2003.04.173

11. Caffo O, Amichetti M, Tomio L, Galligioni E. Quality of life after radiotherapy for early-stage testicular seminoma. Radiother Oncol. 2001;59(1):13-20. doi:10.1016/S0167-8140(00)00264-4

12. Revicki DA, Osoba D, Fairclough D, et al. Recommendations on health-related quality of life research to support labeling and promotional claims in the United States. Qual Life Res. 2000;9(8):887-900. doi:10.1023/A:1008996223999

13. Food and Drug Administration. Guidance for industry: patient-reported outcome measures: use in medical product development to support labelling claims; 2009. Available from: http://www. fda.gov/downloads/Drugs/Guidances/UCM193282.pdf. Accessed May 13, 2021.

14. Rinecones O, Smith AB, Naher SK, Mercieca-Bebber R, Stockler M. An updated systematic review of quantitative studies assessing anxiety, depression, fear of cancer recurrence or psychological distress in testicular cancer survivors. Cancer Manag Res. 2021. 
15. Moher D, Liberati A, Tetzlaff J, Altman DG; The PRISMA Group. Preferred reporting items for systematic reviews and meta-analyses: the PRISMA statement. PLoS Med. 2009;6(7):e1000097. doi:10.1371/journal.pmed.1000097

16. Kmet L, Cook LS, Lee R. Standard quality assessment criteria for evaluating primary research papers from a variety of fields AHFMRHTA initiative20040213. 2004.

17. Aaronson NK, Ahmedzai S, Bergman B, et al. The European organization for research and treatment of cancer QLQ-C30: a quality-oflife instrument for use in international clinical trials in oncology. J Natl Cancer Inst. 1993;85(5):365-376. doi:10.1093/jnci/85.5.365

18. Ware JE, Sherbourne CD. The MOS 36-item short-form health survey (SF-36). I. Conceptual framework and item selection. Med Care. 1992;30(6):473-483. doi:10.1097/00005650-199206000-00002

19. Oldenburg J, Fosså SD, Dahl AA. Scale for chemotherapy-induced long-term neurotoxicity (SCIN): psychometrics, validation, and findings in a large sample of testicular cancer survivors. Qual Life Res. 2006;15(5):791-800. doi:10.1007/s11136-005-5370-6

20. Sztankay M, Aaronson NK, Arraras JI, et al. International Phase IV validation study of an EORTC quality of life questionnaire for testicular cancer patients: the EORTC QLQ-TC26. BMC Cancer. 2018;18 (1):1104. doi:10.1186/s12885-018-5036-8

21. Brorsson B, Ifver J, Hays RD. The Swedish health-related quality of life survey (SWED-QUAL). Qual Life Res. 1993;2(1):33-45. doi:10.1007/BF00642887

22. Sullivan M, Karlsson J, Bengtsson C, Furunes B, Lapidus L, Lissner L. "The Göteborg quality of life instrument"-a psychometric evaluation of assessments of symptoms and well-being among women in a general population. Scand J Prim Health Care. 1993;11(4):267-275. doi:10.3109/02813439308994842

23. Brydoy M, Oldenburg J, Klepp O, et al. Observational study of prevalence of long-term Raynaud-like phenomena and neurological side effects in testicular cancer survivors. J Natl Cancer Inst. 2009;101(24):1682-1695. doi:10.1093/jnci/djp413

24. de Wit R, Roberts JT, Wilkinson PM, et al. Equivalence of three or four cycles of bleomycin, etoposide, and cisplatin chemotherapy and of a 3- or 5-day schedule in good-prognosis germ cell cancer: a randomized study of the European organization for research and treatment of cancer genitourinary tract cancer cooperative group and the medical research council. J Clin Oncol. 2001;19(6):1629-1640. doi:10.1200/JCO.2001.19.6.1629

25. Flechtner HH, Fischer F, Albers P, Hartmann M, Siener R. Quality-of -life analysis of the German prospective multicentre trial of single-cycle adjuvant BEP Versus retroperitoneal lymph node dissection in clinical stage I nonseminomatous germ cell tumours. Eur Urol. 2016;69(3):518-525. doi:10.1016/j.eururo.2015.11.007

26. Fossa SD, de Wit R, Roberts JT, et al. Quality of life in good prognosis patients with metastatic germ cell cancer: a prospective study of the European organization for research and treatment of cancer genitourinary group/Medical research council testicular cancer study group (30941/TE20). J Clin Oncol. 2003;21(6):1107-1118. doi: $10.1200 /$ JCO.2003.02.075

27. Fossa SD, Moynihan C, Serbouti S. Patients' and doctors' perception of long-term morbidity in patients with testicular cancer clinical stage I. A descriptive pilot study. Support Care Cancer. 1996;4 (2):118-128. doi:10.1007/BF01845761

28. Glendenning JL, Barbachano Y, Norman AR, Dearnaley DP, Horwich A, Huddart RA. Long-term neurologic and peripheral vascular toxicity after chemotherapy treatment of testicular cancer. Cancer. 2010;116(10):2322-2331. doi:10.1002/cncr.24981

29. Grimison PS, Stockler MR, Thomson DB, et al. Comparison of two standard chemotherapy regimens for good-prognosis germ cell tumors: updated analysis of a randomized trial. $J$ Natl Cancer Inst. 2010;102(16):1253-1262. doi:10.1093/jnci/djq245
30. Huddart RA, Norman A, Moynihan C, et al. Fertility, gonadal and sexual function in survivors of testicular cancer. $\mathrm{Br} J$ Cancer. 2005;93(2):200-207. doi:10.1038/sj.bjc.6602677

31. Joly F, Heron JF, Kalusinski L, et al. Quality of life in long-term survivors of testicular cancer: a population-based case-control study. J Clin Oncol. 2002;20(1):73-80. doi:10.1200/JCO.2002.20.1.73

32. Jones WG, Fossa SD, Mead GM, et al. Randomized trial of 30 versus $20 \mathrm{~Gy}$ in the adjuvant treatment of stage I testicular seminoma: a report on medical research council trial TE18, European organisation for the research and treatment of cancer trial 30942 (ISRCTN18525328). $J$ Clin Oncol. 2005;23(6):1200-1208. doi:10.1200/JCO.2005.08.003

33. Jonker-Pool G, van Basten JP, Hoekstra HJ, et al. Sexual functioning after treatment for testicular cancer: comparison of treatment modalities. Cancer. 1997;80(3):454-464. doi:10.1002/(SICI)10970142(19970801)80:3<454::AID-CNCR13>3.0.CO;2-W

34. Kerns SL, Fung C, Monahan PO, et al. Cumulative burden of morbidity among testicular cancer survivors after standard cisplatin-based chemotherapy: a multi-institutional study. J Clin Oncol. 2018;36(15):1505-1512. doi:10.1200/JCO.2017.77.0735

35. Kim C, McGlynn KA, McCorkle R, et al. Quality of life among testicular cancer survivors: a case-control study in the United States. Qual Life Res. 2011;20(10):1629-1637. doi:10.1007/s11136-0119907-6

36. Miyake H, Muramaki M, Eto H, Kamidono S, Hara I. Health-related quality of life after chemotherapy for advanced germ cell tumors: a comparison of standard-dose and high-dose chemotherapy. Int J Urol. 2004;11(7):542-546. doi:10.1111/j.1442-2042.2004.00839.x

37. Mykletun A, Dahl AA, Haaland CF, et al. Side effects and cancer-related stress determine quality of life in long-term survivors of testicular cancer. J Clin Oncol. 2005;23(13):3061-3068. doi: $10.1200 / \mathrm{JCO} .2005 .08 .048$

38. Oechsle K, Hartmann M, Mehnert A, Oing C, Bokemeyer C, Vehling S. Symptom burden in long-term germ cell tumor survivors. Support Care Cancer. 2016;24(5):2243-2250. doi:10.1007/s00520-015-3026-9

39. Rossen PB, Pedersen AF, Zachariae R, von der Maase H. Health-related quality of life in long-term survivors of testicular cancer. J Clin Oncol. 2009;27(35):5993-5999. doi:10.1200/JCO.2008.19.6931

40. Schmidt AH, Hoyer M, Jensen BFS, Agerbaek M. Limited post-chemotherapy retroperitoneal resection of residual tumour in non-seminomatous testicular cancer: complications, outcome and quality of life. Acta Oncol. 2018;57(8):1084-1093. doi:10.1080/ 0284186X.2018.1449249

41. Skaali T, Fossa SD, Andersson S, et al. Self-reported cognitive problems in testicular cancer patients: relation to neuropsychological performance, fatigue, and psychological distress. J Psychosom Res. 2011;70(5):403-410. doi:10.1016/j.jpsychores.2010.12.004

42. Skalleberg J, Smastuen MC, Oldenburg J, Osnes T, Fossa SD, Bunne M. The relationship between cisplatin-related and age-related hearing loss during an extended follow-up. Laryngoscope. 2020;17:17.

43. Skoogh J, Steineck G, Stierner U, et al. Testicular-cancer survivors experience compromised language following chemotherapy: findings in a Swedish population-based study 3-26 years after treatment. Acta Oncol. 2012;51(2):185-197. doi:10.3109/0284186X.2011.602113

44. Sprauten M, Darrah TH, Peterson DR, et al. Impact of long-term serum platinum concentrations on neuro- and ototoxicity in Cisplatin-treated survivors of testicular cancer. $J$ Clin Oncol. 2012;30(3):300-307. doi:10.1200/JCO.2011.37.4025

45. Vidrine DJ, Hoekstra-Weebers JE, Hoekstra HJ, Tuinman MA, Marani S, Gritz ER. The effects of testicular cancer treatment on health-related quality of life. Urology. 2010;75(3):636-641. doi:10.1016/j.urology.2009.09.053 
46. Whitford HS, Kalinowski P, Schembri A, et al. The impact of chemotherapy on cognitive function: a multicentre prospective cohort study in testicular cancer. Support Care Cancer. 2019.

47. Wortel RC, Ghidey Alemayehu W, Incrocci L. Orchiectomy and radiotherapy for stage I-II testicular seminoma: a prospective evaluation of short-term effects on body image and sexual function. $J$ Sex Med. 2015;12(1):210-218. doi:10.1111/jsm.12739

48. Fung C, Sesso HD, Williams AM, et al. Multi-institutional assessment of adverse health outcomes among North American testicular cancer survivors after modern cisplatin-based chemotherapy. J Clin Oncol. 2017;35(11):1211-1222. doi:10.1200/JCO.2016.70.3108

49. Dearnaley DP, Fossa SD, Kaye SB, et al. Adjuvant bleomycin, vincristine and cisplatin (BOP) for high-risk stage I non-seminomatous germ cell tumours: a prospective trial (MRC TE17). $B r \quad J$ Cancer. 2005;92(12):2107-2113. doi:10.1038/sj. bjc. 6602624

50. Hanna N, Einhorn LH. Testicular cancer: a reflection on 50 years of discovery. J Clin Oncol. 2014;32(28):3085-3092. doi:10.1200/ JCO.2014.56.0896

51. National Comprehensive Cancer Network. NCCN guidelines for testicular cancer version $1.2021 ; 2020$. Available from: https://www. nccn.org/professionals/physician_gls/pdf/testicular.pdf. Accessed May 13, 2021.

52. Moher D, Pham B, Klassen TP, et al. What contributions do languages other than English make on the results of meta-analyses? $J$ Clin Epidemiol. 2000;53(9):964-972. doi:10.1016/S0895-4356(00) 00188-8
53. Bottomley A, Pe M, Sloan J, et al. Analysing data from patient-reported outcome and quality of life endpoints for cancer clinical trials: a start in setting international standards. Lancet Oncol. 2016;17(11):e510-e4. doi:10.1016/S1470-2045(16)30510-1

54. Calvert M, Kyte D, Mercieca-Bebber R, et al. Guidelines for inclusion of patient-reported outcomes in clinical trial protocols: the spirit-pro extension. JAMA. 2018;319(5):483-494. doi:10.1001/ jama.2017.21903

55. Mercieca-Bebber R, Palmer MJ, Brundage M, Calvert M, Stockler MR, King MT. Design, implementation and reporting strategies to reduce the instance and impact of missing patient-reported outcome (PRO) data: a systematic review. BMJ Open. 2016;6(6). doi:10.1136/bmjopen-2015-010938

56. Coens C, Pe M, Dueck AC, et al. International standards for the analysis of quality-of-life and patient-reported outcome endpoints in cancer randomised controlled trials: recommendations of the SISAQOL Consortium. Lancet Oncol. 2020;21(2):e83-e96. doi:10.1016/S1470-2045(19)30790-9

57. Calvert M, Brundage M, Jacobsen PB, Schunemann HJ, Efficace F. The CONSORT patient-reported outcome (PRO) extension: implications for clinical trials and practice. Health Qual Life Outcomes. 2013;11:184. doi:10.1186/1477-7525-11-184

\section{Publish your work in this journal}

Patient Related Outcome Measures is an international, peer-reviewed, open access journal focusing on treatment outcomes specifically relevant to patients. All aspects of patient care are addressed within the journal and practitioners from all disciplines are invited to submit their work as well as healthcare researchers and patient support groups.
The manuscript management system is completely online and includes a very quick and fair peer-review system. Visit http://www. dovepress.com/testimonials.php to read real quotes from published authors. 